
Agricultures tropicales en poche

Directeur de la collection

Philippe Lhoste

\section{Le palmier à huile en plantation villageoise}

Jean-Charles Jacquemard

Éditions Quæ, CTA, Presses agronomiques de Gembloux 
Le Centre technique de coopération agricole et rurale (CTA) est une institution internationale conjointe des États du Groupe ACP (Afrique, Caraïbes, Pacifique) et de l'Union européenne (UE). Il intervient dans les pays ACP pour améliorer la sécurité alimentaire et nutritionnelle, accroître la prospérité dans les zones rurales et garantir une bonne gestion des ressources naturelles. Il facilite l'accès à l'information et aux connaissances, favorise l'élaboration des politiques agricoles dans la concertation et renforce les capacités des institutions et communautés concernées.

Le CTA opère dans le cadre de l'Accord de Cotonou et est financé par l'UE.

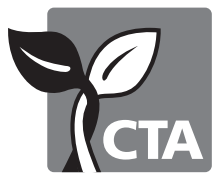

CTA, Postbus 380, 6700 AJ Wageningen, Pays-Bas

www.cta.int

Éditions Quæ, RD 10, 78026 Versailles Cedex, France

www.quae.com

Presses agronomiques de Gembloux, Passage des Déportés, 2,

B-5030 Gembloux, Belgique

www.pressesagro.be

(C) Quæ, CTA, Presses agronomiques de Gembloux 2013

ISBN (Quæ) : 978-2-7592-1987-2

ISBN (CTA) : 978-92-9081-526-6

ISBN (PAG) : 978-2-87016-127-2

ISSN : $1778-6568$

(C) Le code de la propriété intellectuelle du $1^{\text {er }}$ juillet 1992 interdit la photocopie à usage collectif sans autorisation des ayants droit. Le non-respect de cette disposition met en danger l'édition, notamment scientifique. Toute reproduction, partielle ou totale, du présent ouvrage est interdite sans autorisation des éditeurs ou du Centre français d'exploitation du droit de copie (CFC), 20, rue des Grands-Augustins, 75006 Paris. 


\section{Table des matières}

Avant-propos .........................................

Remerciements...................................

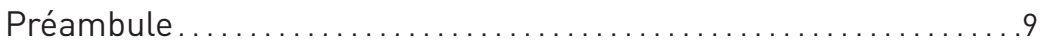

Calendrier cultural général ........................... 11

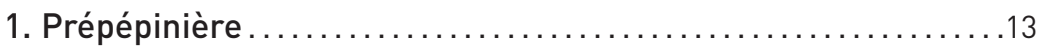

Fiche 1. Préparation de la prépépinière........................ 14

Fiche 2. Choix du terreau et remplissage des sachets ............... 16

Fiche 3. Mise en place des sachets et de l'ombrière................ 18

Fiche 4 . Tri et repiquage des graines germées ................... 20

Fiche 5. Entretien de la prépépinière ....................... 23

Fiche 6. Problèmes rencontrés dans la prépépinière ................ 25

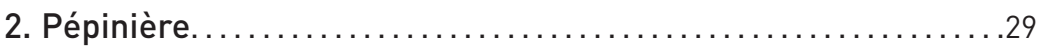

Fiche 7. Choix et préparation de l'emplacement .................. 30

Fiche 8. Choix du terreau et remplissage des sacs ................. 32

Fiche 9. Mise en place des sacs et de l'ombrière................... 34

Fiche 10. Sélection des plantules .......................... 36

Fiche 11. Transport et repiquage des plantules en pépinière ........... 38

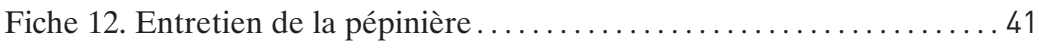

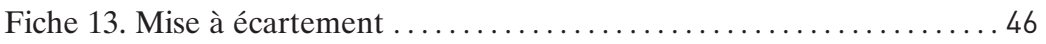

Fiche 14. Sélection des plants ............................. 49

Fiche 15. Problèmes rencontrés dans la pépinière ................. 51

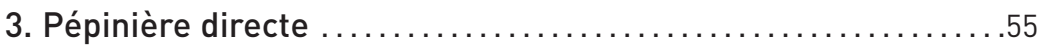

Fiche 16. Entretien de la pépinière directe..................... 56

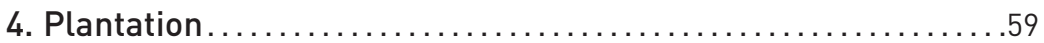

Fiche 17. Localisation de la parcelle.........................60 60

Fiche 18. Préparation du terrain sur forêt dégradée ou vieille jachère ....61 61

Fiche 19. Préparation du terrain pour une replantation

derrière palmeraie....................................... 64

Fiche 20. Fabrication et utilisation du gabarit d'angle droit ............66 66

Fiche 21. Délimitation de la parcelle et ligne de base dans le cas d'une extension sur forêt.........................68

Fiche 22. Piquetage des lignes de plantation dans le cas d'une extension sur forêt. ......................... 71 
Fiche 23. Piquetage des palmiers dans le cas d'une extension sur forêt . . . 73 Fiche 24. Piquetage des lignes et emplacement des arbres pour une replantation derrière palmeraie . . . . . . . . . . . . . . . 75

Fiche 25. Dégagement des lignes de palmiers . . . . . . . . . . . . 77

Fiche 26. Mise en place des plants. . . . . . . . . . . . . . . . . . 79

5. Jeunes cultures.................................... 83

Fiche 27. Entretien de la parcelle : ronds et couverture............ 84

Fiche 28. Extirpation de Chromolaena odorata . . . . . . . . . . . . 87

Fiche 29. Cultures intercalaires et élevage ..................... 90

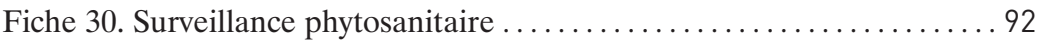

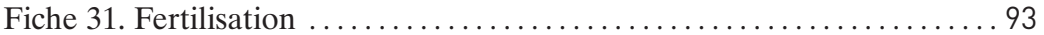

6. Palmiers en exploitation $\ldots \ldots \ldots \ldots \ldots \ldots \ldots \ldots \ldots \ldots \ldots \ldots \ldots$

Fiche 32 . Entretien des ronds . . . . . . . . . . . . . . . . . . . 98

Fiche 33. Entretien des interlignes . . . . . . . . . . . . . . . 100

Fiche 34 . Entrée en production ............................ 102

Fiche 35. Élagage ..................................... 104

Fiche 36. Récolte des régimes................................ 106

Fiche 37. Techniques de récolte . . . . . . . . . . . . . . . . . . 109

Fiche 38. Fumure. .................................... 113

Fiche 39. Épandage de rafles. ......................... 116

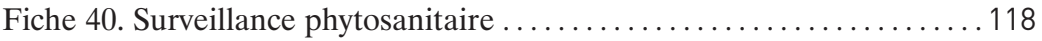

7. Défense des cultures .......................... 119

Fiche 41. Principales maladies rencontrées en Afrique de l'Ouest et en Afrique équatoriale ................................ 121

Fiche 42. Principaux ravageurs rencontrés en Afrique de l'Ouest et en Afrique équatoriale ............................... 124

Fiche 43. Déficiences nutritionnelles et dégâts provoqués par la foudre . 129

8. Sécurité ........................................ 133

Fiche 44. Rappels de sécurité ............................ 133

Glossaire......................................... 137

Pour en savoir plus .................................. 141

Cahier couleur.................................. 120 


\section{Avant-propos}

La collection «Agricultures tropicales en poche» (ATP) a été créée par un consortium comprenant le CTA de Wageningen (Pays-Bas), les Presses agronomiques de Gembloux (Belgique) et les Éditions Quæ (France). Elle comprend trois séries d'ouvrages pratiques consacrés aux productions animales, aux productions végétales et aux questions transversales.

Ces guides pratiques sont destinés avant tout aux producteurs, aux techniciens et aux conseillers agricoles et c'est tout particulièrement le cas de ce nouvel ouvrage de Jean-Charles Jacquemard. Cet auteur a en effet produit, fin 2011, dans cette même collection ATP, un premier ouvrage sur le palmier à huile; dans cet ouvrage, l'auteur fournissait aux praticiens les recommandations et informations nécessaires pour la mise en place et l'exploitation d'une palmeraie commerciale. Dans ce nouvel ouvrage sur la même plante, le palmier à huile, il s'adresse cette fois aux petits planteurs villageois. Ce nouveau guide pratique est en effet adapté aux conditions des petites exploitations familiales. Il a pour objectif d'aider ces agriculteurs à bien maîtriser tous les aspects techniques et pratiques de la création et du développement de leur plantation de palmier à huile.

Cet ouvrage est rédigé de façon très didactique et remarquablement illustré. Ce guide organisé en 44 fiches regroupées en huit chapitres qui traitent de chaque stade de l'itinéraire technique : de la prépépinière et la création de la palmeraie, à l'entretien de la plantation en production et à la récolte des régimes; il aborde aussi, in fine, les questions de sécurité qui sont importantes pour une telle production tant au plan des équipements et des outils utilisés qu'à celui des produits phytosanitaires manipulés. Les fiches qui abordent des aspects opératoires sont illustrées de nombreuses photos très précises qui montrent clairement les manipulations décrites. Les principaux symptômes de maladies ou de problèmes à tous les stades de la plantation sont décrits et illustrés de façon très explicite. Lauteur apporte aussi des réponses pratiques aux questions et aux problèmes que les planteurs peuvent rencontrer aux différents stades de leur entreprise.

Cet «ouvrage-compagnon», consacré au palmier à huile et dédié aux petits planteurs, complète donc de façon très pragmatique le premier ouvrage de la collection ATP sur cette même plante. Les deux livres sont en fait complémentaires, ils n'abordent pas cette culture de la même façon, ils ne visent pas exactement le même public. 
Lintérêt de cet ouvrage se justifie pleinement pour deux raisons principales. D'abord le palmier à huile est une culture très importante aux plans économique et alimentaire dans les régions tropicales humides et il constitue la principale source de corps gras végétaux sur le marché mondial. Ensuite, la proportion des superficies de palmeraie exploitées par ces petits planteurs est variable selon les pays mais loin d'être négligeable : elle peut atteindre plus de $50 \%$ dans des pays comme le Cameroun, la Côte d'Ivoire, le Ghana, le Nigeria, la Sierra Leone ou la Thaillande.

Ce nouveau guide très pratique est particulièrement destiné aux planteurs villageois et à leurs encadreurs; il doit leur permettre d'intégrer dans leurs pratiques quotidiennes, les gestes et les techniques les plus adaptés au développement durable de leur plantation de palmier à huile.

Lauteur, Jean-Charles Jacquemard, est un spécialiste de cette culture à laquelle il a consacré toute sa carrière en recherche-développement sur le terrain dans différents pays d'Afrique et d'Asie.

Philippe Lhoste Directeur de la collection Agricultures tropicales en poche 


\section{Remerciements}

Je voudrais remercier les Éditions Quæ, les Presses agronomiques de Gembloux et le CTA pour avoir accepté de publier ce projet qui me tenait à cœur depuis de nombreuses années et qui est un complément indispensable au livre «Le Palmier à huile» comme Philippe Lhoste le souligne dans son avant-propos.

Que Bertrand Tailliez, qui fut le gardien attentif du manuscrit original et des illustrations et qui a bien voulu faire une lecture attentive et technique de ce manuscrit, soit particulièrement remercié.

Merci aussi à Marie-Aline Jacquemard dont la relecture m'a aidé, une fois encore, à améliorer la lisibilité et la compréhension d'un ouvrage destiné au public si particulier que sont les petits planteurs.

Que les éditrices, Claire Jourdan-Ruf, Claire Parmentier et Martine Séguier-Guis, le directeur de la collection Agricultures tropicales en poche, Philippe Lhoste, ainsi que leurs collaborateurs soient également remerciés pour le travail accompli dans la mise en forme finale de cet ouvrage.

Une dédicace toute particulière est adressée à Baudelaire Housinou Sourou, ancien secrétaire exécutif de l'Association africaine pour le Développement du palmier à huile, pour le soutien actif et permanent qu'il avait apporté aux prémices d'un projet qui lui tenait aussi très à cœur.

Enfin, j'associe à ces remerciements, mon épouse, Jeannine, pour son soutien constant et son indulgence devant les nombreuses heures passées à mettre en forme ce manuscrit.

Jean-Charles Jacquemard 



\section{$\checkmark$ Préambule}

Lextraordinaire développement de la place de l'huile de palme dans le marché mondial des corps gras à la fin du $\mathrm{xx}^{\mathrm{e}}$ siècle et pendant la première décennie du $\mathrm{XXI}^{\mathrm{e}} \mathrm{s}$ 'est traduit par la plantation de plus de 12 millions d'hectares de palmeraies. Même si ce développement concerne 43 pays tropicaux, l'essentiel de celui-ci s'est concentré en Asie du Sud-Est (Malaisie, Indonésie, Papouasie-Nouvelle Guinée et Thaiilande). La Malaisie et l'Indonésie, notamment, incluent dans leurs politiques de développement un objectif de $10 \%$ d'agrocarburants à base d'huile de palme dans leur consommation nationale.

Ce développement a été plus souvent basé sur une extension des superficies que sur une augmentation des rendements en huile par hectare. Le succès de la filière palmier à huile a par conséquent fait l'objet de vives critiques quant à son impact sur l'environnement, la déforestation et les populations locales.

Ces dernières années, les projets de développement de la culture semblent s'être déplacés de l'Asie du Sud-Est vers l'Afrique de l'Ouest et vers l'Afrique équatoriale, avec le même cortège de critiques mettant en cause l'agro-business du palmier à huile.

Il convient malgré tout de ne pas oublier qu'en marge de cet agrobusiness, s'est développé toute une galaxie de petites exploitations dont la taille varie de 1 à 40 ha. Le tableau 1 montre que la proportion des superficies occupées par les petites exploitations est loin d'être négligeable. En Asie du Sud-Est, la proportion de ce type d'exploitation varie de 41 à $76 \%$. Dans les pays de vieille tradition élaéicole en Afrique, aux superficies occupées par les petites exploitations, il faut ajouter la mise en valeur de la palmeraie naturelle qui occupe plus d'un million d'hectares comme au Nigeria ou en République démocratique du Congo.

En Amérique du Sud, notamment en Colombie, au Pérou ou en Équateur, le développement de petites exploitations de palmier à huile a pour objectif d'être une alternative économiquement viable à la culture de la coca.

Ces petits exploitants peuvent être encadrés soit par une structure d'État, soit par une coopérative, soit par une plantation commerciale. Près des deux tiers des petits exploitants de Malaisie et d'Indonésie est encadré, alors que ce pourcentage chute fortement dans certains pays d'Afrique où la structure d'encadrement est soit inexistante, soit 
s'est considérablement affaiblie après la privatisation des plantations à capitaux publics, les tensions politiques, les crises économiques récurrentes, voire les guerres civiles.

Tableau 1. Les petites exploitations productrices de palmier à huile (entre 1 et 40 ha dans les pays producteurs d'huile de palme).

\begin{tabular}{lll}
\hline Continent & Pays & \% de petites exploitations \\
\hline Asie du Sud-Est & Indonésie & 44 \\
\cline { 2 - 3 } & Malaisie & 41 \\
\cline { 2 - 3 } & Papouasie-Nouvelle Guinée & 42 \\
\cline { 2 - 3 } & Thaïlande & 76 \\
\hline \multirow{2}{*}{ ffrique } & Nigeria & 80 \\
\cline { 2 - 3 } & Côte d'Ivoire & 70 \\
\cline { 2 - 3 } & Ghana & 88 \\
\cline { 2 - 3 } & Cameroun & 59 \\
\cline { 2 - 3 } & Sierra Leone & 62 \\
\cline { 2 - 3 } & Liberia (projets) & 16 \\
\hline Amérique du Sud & Colombie &
\end{tabular}

Aussi, en complément du livre «Le palmier à huile», nous avons voulu présenter les différents itinéraires techniques qui seront utiles à un petit planteur pour créer ou développer sa plantation de palmier à huile, ainsi qu'à l'aider à en tirer le meilleur profit. 


\section{Calendrier cultural général}

Ce calendrier présente les principales opérations relatives à la culture du palmier à huile. Les informations et données techniques sont calibrées pour une plantation de 5 hectares de palmier à huile.

Tableau 2. Calendrier cultural général pour un climat à grande saison des pluies d'avril à juillet.

\begin{tabular}{lcc}
\hline Opération & Mois & Année \\
\hline Commande des graines germées & janvier & -1 \\
\hline Repiquage des graines germées & mai & -1 \\
\hline Préparation de la pépinière & août & -1 \\
\hline Repiquage des plantules en pépinière & septembre & -1 \\
\hline Début de la préparation du terrain & septembre & -1 \\
\hline Plantation & mai-juin & 1 \\
\hline
\end{tabular}

Par convention, l'année 1 commence à la date de plantation et s'achève l'année suivante à la date anniversaire de cette plantation. Lannée 2 commence à la date du premier anniversaire de la plantation pour s'achever à la date anniversaire de l'année suivante. Il en est de même pour les années suivantes. 



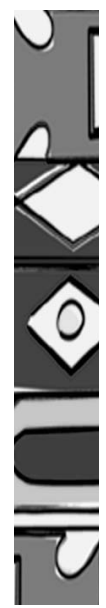

\section{Prépépinière}

La prépépinière correspond à la culture du palmier à huile pendant les quatre mois qui suivent la germination. Pendant cette période, la jeune plante passe par les étapes suivantes :

- la graine germée est repiquée avec son coléoptile et une radicule;

- les deux feuilles primordiales et des racines adventives sont émises au cours du premier mois;

- une première feuille lancéolée apparaît ensuite, ainsi qu'une première racine primaire;

- à 4 mois, la plantule présente 3 à 4 feuilles lancéolées et un système racinaire bien développé avec des racines primaires, secondaires et tertiaires; la plantule est repiquée en pépinière lorsque la troisième feuille est bien épanouie.

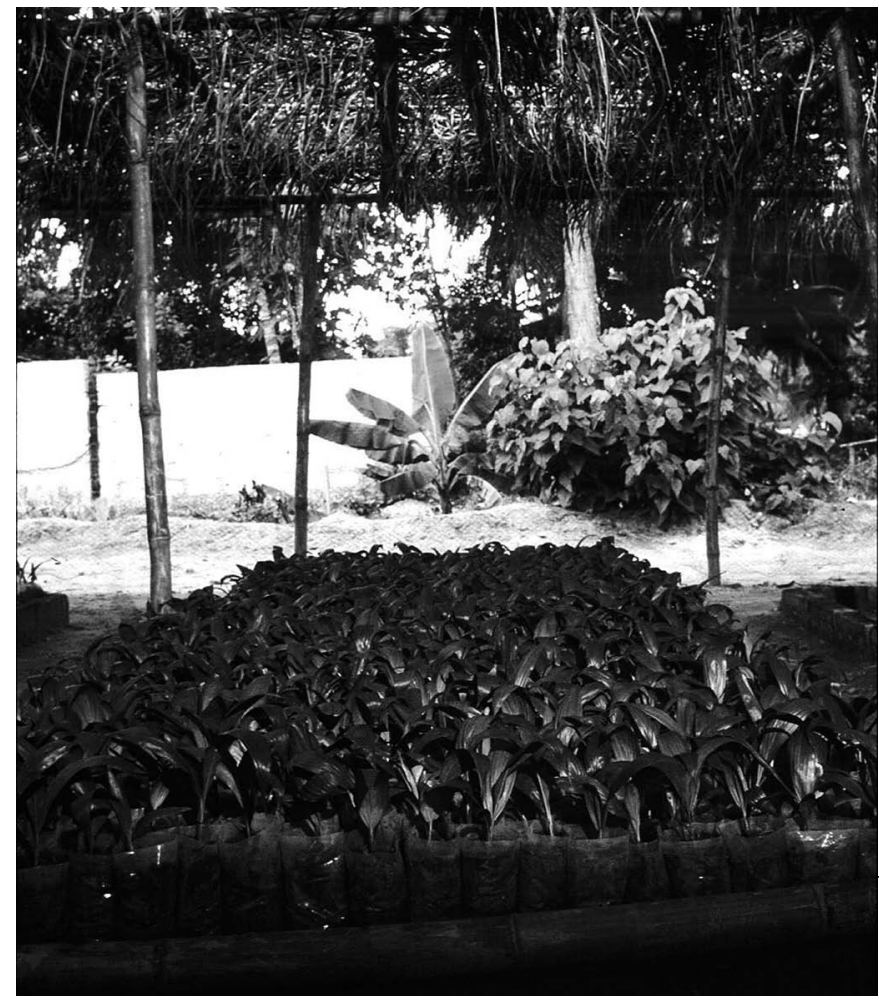

Figure 1.

Prépépinière en place. 


\section{Fiche 1. Préparation de la prépépinière}

\section{ID Matériel et produits nécessaires}

- 1 machette lourde

- 1 houe ou une daba

- $19 \mathrm{~m}$ de grillage à poule d'1 $\mathrm{m}$ de largeur

- Bambous pour les poteaux, les traverses, les bords de la planche et la barrière de protection

- Xylophène, sulfate de cuivre ou 1 à 2 litres d'huile de vidange

- 1 pinceau plat de $5 \mathrm{~cm}$ de large ou équivalent.

\section{ID Choix de l'emplacement}

Pour une bonne surveillance, la prépépinière doit être :

- proche de l'habitation;

- proche d'un point d'eau.

Le terrain doit être en pente légère, sans eau stagnante et le sol ni trop argileux, ni trop gravillonnaire.

\section{ID Préparation de l'emplacement}

Désherber à la houe un emplacement de 5,5 x $8 \mathrm{~m}$.

Délimiter au centre une planche de 1,5 x 3,75 m. Marquer les limites de la planche avec des bambous coupés à la bonne longueur maintenus par des petits piquets (figure 1.1).

Couper 6 gros bambous de 2,5 $\mathrm{m}$ de long.

Entailler une extrémité en $\mathrm{V}$ et traiter l'autre extrémité sur $50 \mathrm{~cm}$ avec du xylophène, du sulfate de cuivre ou de l'huile de vidange.

Creuser 6 trous de $50 \mathrm{~cm}$ de profondeur à $1 \mathrm{~m}$ de distance de la planche, vers les coins et vers son milieu.

Enfoncer les bambous dans les trous, « $\mathrm{V} »$ en haut.

Placer deux traverses en bambou plus léger longitudinalement sur les poteaux, puis sept traverses perpendiculairement tous les mètres approximativement.

Sur les poteaux, poser un grillage de protection de $1 \mathrm{~m}$ de hauteur et enfoncé de $15 \mathrm{~cm}$ dans le sol. Il peut être remplacé par une rangée d'éclats de bambous, de rachis de feuille de palmier ou de raphia. 
Autour du grillage, faire un petit fossé de $20 \mathrm{~cm}$ de profondeur et $40 \mathrm{~cm}$ de large.

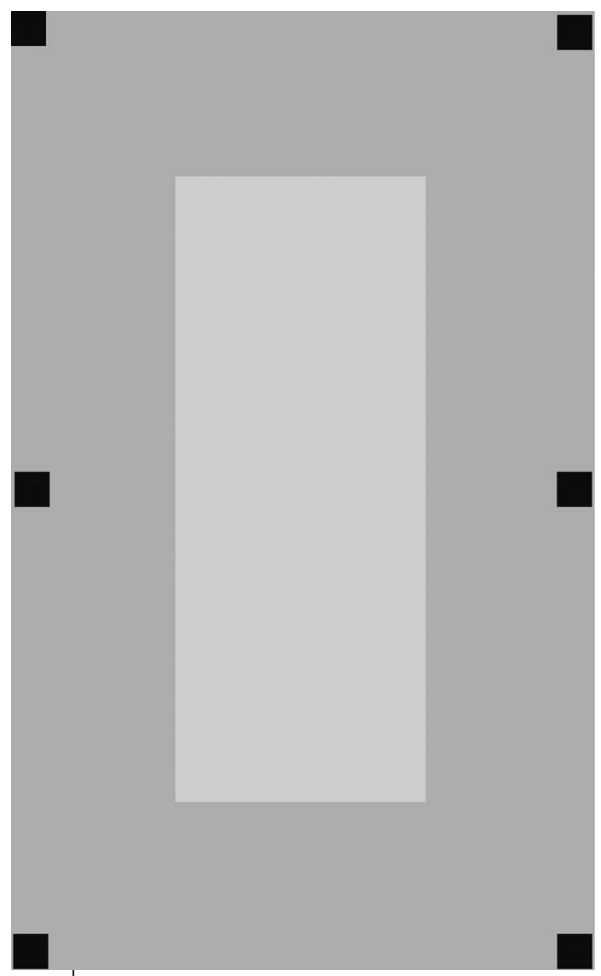

Figure 1.1.

Schéma de la prépépinière.

Au centre : planche de 1,5 m x 3,75 m. Autour : emprise de l'ombrière qui déborde d'1 $\mathrm{m}$ au-delà de la planche. Position des 6 trous et poteaux de support de l’ombrière. 


\section{Fiche 2. Choix du terreau et remplissage des sachets}

\section{Matériel nécessaire et temps de travail}

- 1 pelle de terrassier

- 1 à 3 tines vides $(1$ tine $=$ un container de récupération d'environ 25 litres de capacité)

- 1 bâche plastique noire d'environ $10 \mathrm{~m}^{2}$

- 1 tamis en grillage à maille de 1 à $2 \mathrm{~cm}$

- 1000 sachets de plastique transparent ou noir

- 1 tonne de terreau ou de mélange de terreau + compost

- Nombre de journées de travail nécessaire : 5 à 6 HJ (homme-jour : unité de mesure correspondant au travail d'une personne pendant un jour, la durée du jour de travail étant fixée par la loi ou par un accord d'entreprise).

\section{ID Choix du terreau}

Prendre du terreau de forêt prélevé dans les 10 premiers centimètres de sol, de préférence léger et humifère.

Passer le terreau sur un tamis en grillage à maille de 1 à $2 \mathrm{~cm}$ (figure 2.1).

Si cela est possible :

- tamiser des déchets domestiques et agricoles bien décomposés pour obtenir du compost (figure 2.2);

- mélanger $1 / 3$ de compost ( 1 tine) et $2 / 3$ de terreau ( 2 tines) à la pelle de terrassier;

- protéger le mélange ou le terreau avec une bâche en plastique noir.

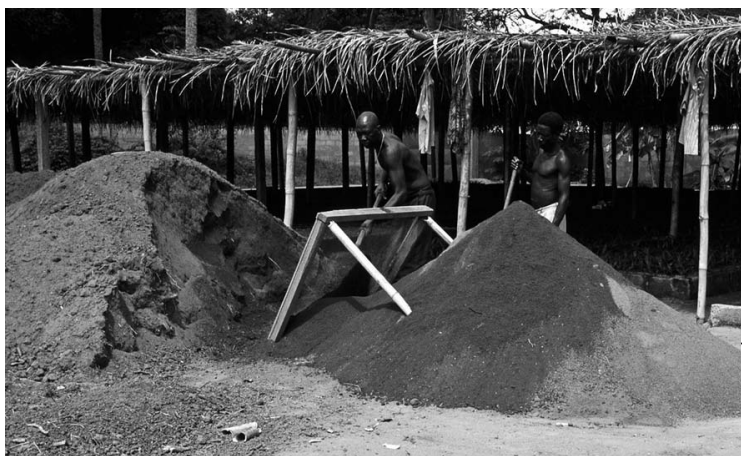

Figure 2.1.

Chantier

de tamisage.

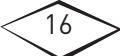




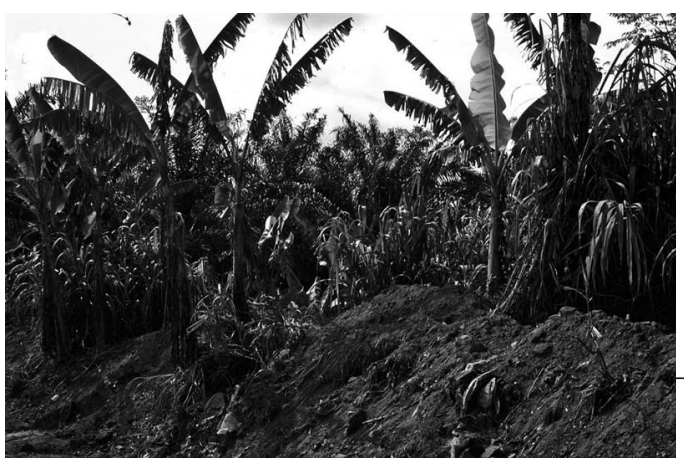

\section{Figure 2.2.}

Compost de village.

\section{ID Caractéristiques des sachets à vide}

- Matériau : polyéthylène transparent ou noir, à soufflets

- Épaisseur : 0,05 mm

- Largeur : 8,5 cm

- Hauteur : $20 \mathrm{~cm}$

- Base perforée de 20 trous de $5 \mathrm{~mm}$ de diamètre

- Pour 5 hectares de plantation, il faut 1000 sachets.

\section{ID Remplissage des sachets}

Remplir les sachets jusqu'à $1 \mathrm{~cm}$ du bord supérieur en tassant modérément la terre (figures 2.3 et 2.4).

Il faut environ $1 \mathrm{~kg}$ de terreau pour remplir 1 sachet.

Pour 1000 sachets, il faut 1 tonne de terreau soit 33 à 35 tines.

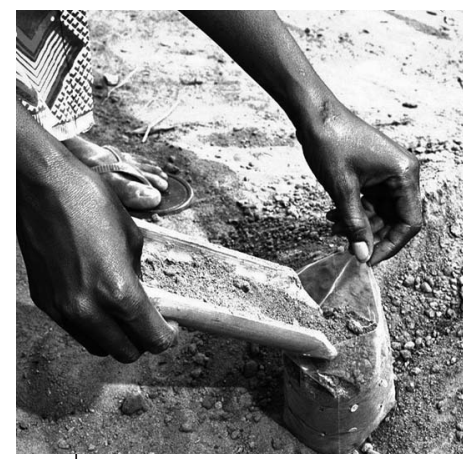

Figure 2.3.

Remplissage des sachets.

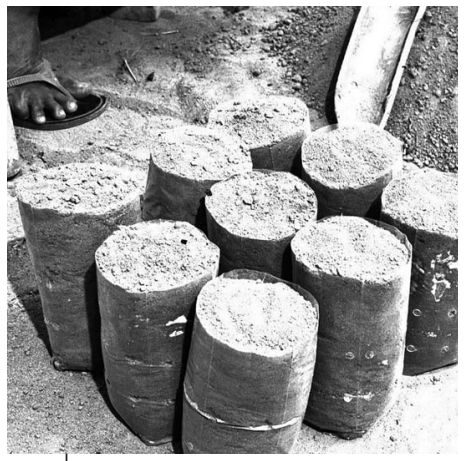

Figure 2.4.

Sachets bien remplis. 


\section{Fiche 3. Mise en place des sachets et de l'ombrière}

ID Matériel et produits nécessaires

- $3 \mathrm{~g}$ de sulfate neutre d'oxyquinoléine à $0,1 \%$

- 0,6 g de deltaméthrine ou 3 litres d'eau de Javel à 2,6\%

- 1 arrosoir de 10 litres avec pomme

- 20 feuilles de palmier ou de raphia à remplacer après 2 mois

- De l'eau propre (100 litres environ).

\section{ID Mise en place des sachets}

Le plus tôt possible après le remplissage :

- installer les sachets dans la planche en lignes de 20 (figure 3.1);

- désinfecter le terreau.

Le traitement du terreau est effectué de la façon suivante :

- soit avec une solution contenant $1 \mathrm{~g}$ de sulfate neutre d'oxyquinoléine à $0,1 \%$ et $0,2 \mathrm{~g}$ de deltaméthrine dans 10 litres d'eau;

- soit avec une solution contenant 1 litre d'eau de Javel à 2,6\% dans

10 litres d'eau;

- pour 1000 sachets il faut 3 arrosoirs de 10 litres;

- ensuite arroser abondamment pour bien mouiller le terreau jusqu'au fond du sachet.

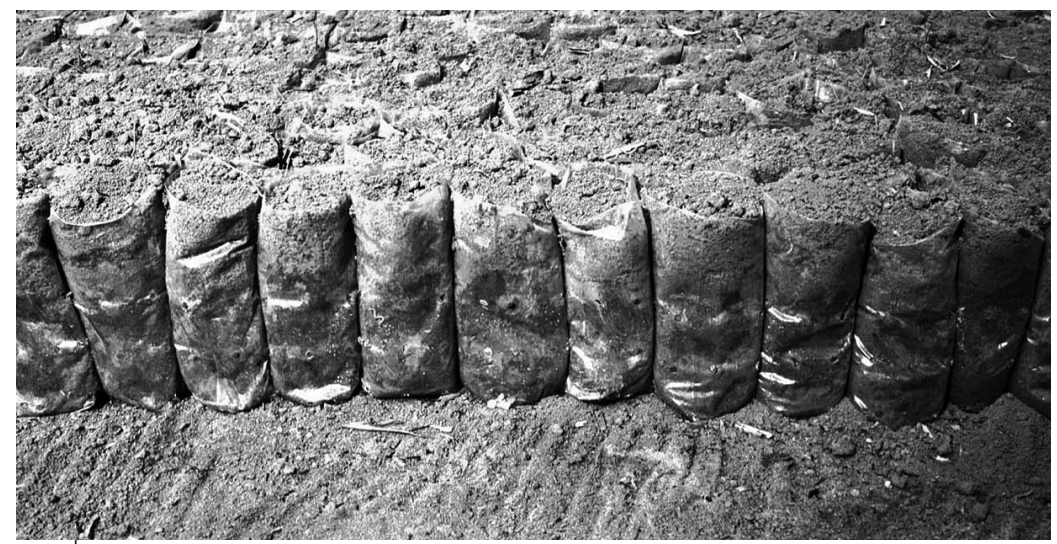

Figure 3.1.

Sachets mis en place. 


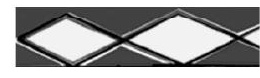

\section{Mise en place de l'ombrière}

Poser des feuilles de palmier ou de raphia sans leur pétiole perpendiculairement aux traverses (figure 3.2).

Compter 3 à 4 feuilles par mètre courant de traverse, soit environ 20 feuilles au total.

Remplacer les feuilles tous les 2 mois.

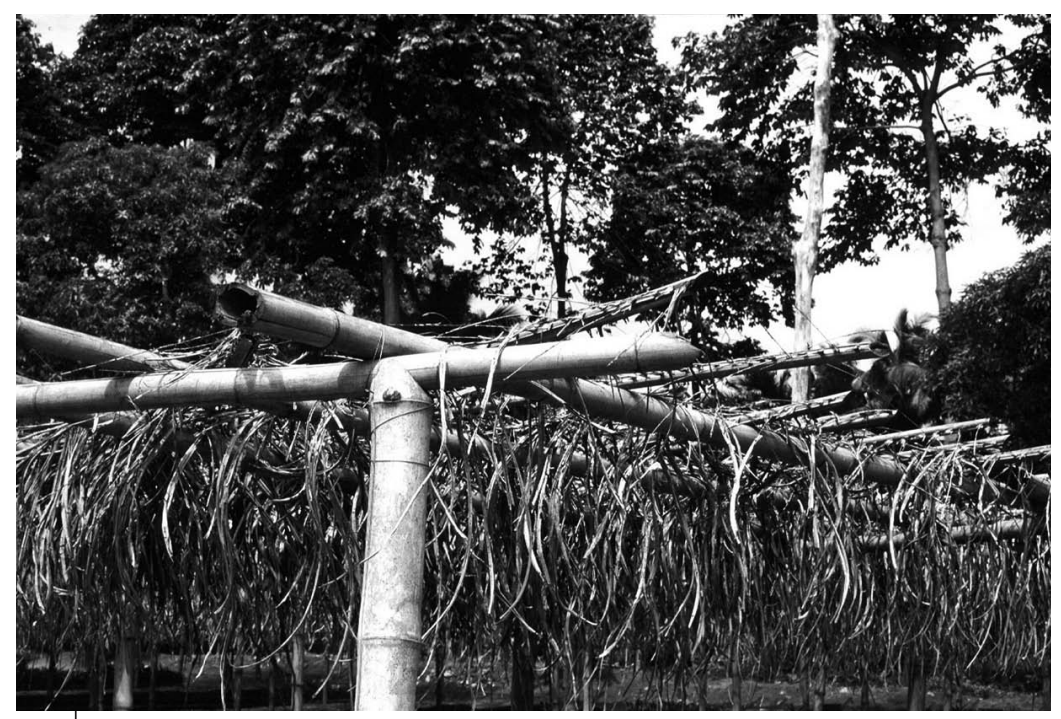

Figure 3.2.

Ombrière mise en place. 


\section{Fiche 4. Tri et repiquage des graines germées}

\section{Matériel nécessaire}

- 1 plateau propre

- 1 seau avec de l'eau claire pour se rincer les mains.

\section{Tri des graines germées}

Le planteur doit se fournir auprès d'un Centre agréé qui certifie la légitimité des semences et le potentiel de production futur des palmiers. Il lui faut 1000 graines germées.

Les graines qui germent spontanément au pied des palmiers sont totalement impropres à la réalisation d'une plantation de qualité. Les palmiers qui en seront issus auront une forte proportion d'anormaux, environ la moitié seront totalement ou très faiblement productifs (pisifera et dura) ou très consanguins (tenera).

Les graines sont conservées au maximum 8 jours dans une pièce sombre à l'abri de la lumière et de toute source de chaleur, des souris ou des rats. Les graines germées ont été mélangées le plus souvent à des billes de polystyrène et placées dans des sacs en plastique. Si les graines sont trop sèches, il convient de les humecter légèrement avant de les trier.

Cette opération de tri se fait la veille du jour prévu pour le repiquage en fin d'après-midi.

Renverser le contenu d'un des sacs sur un plateau propre, par exemple un plateau à vanner.

Séparer les graines ayant un germe différencié de celles qui n'en ont pas. Le germe différencié normal présente un coléoptile et une radicule de couleur ivoire dont la longueur totale est de 8 à $15 \mathrm{~mm}$ (figure 4.2).

Éliminer les graines germées anormales.

Seules les graines présentant un germe différencié normal seront repiquées.

Remettre les graines non différenciées dans le sac de plastique (figure 4.1).

Refermer le sac.

Remettre le sac à l'abri.

Conserver les graines germées anormales dans un sac en plastique. 
Repiquer immédiatement les graines germées différenciées.

Après une semaine, reprendre la même série d'opérations sur les graines germées non repiquées.

Si à la fin des opérations, il y a plus de 30 graines germées anormales (sur les 1000 ), contacter le centre de production pour une réclamation et une compensation.

\section{ID Repiquage des graines germées}

Arroser légèrement les sachets de la prépépinière la veille du repiquage. Commencer les opérations au lever du jour.

Déposer une à une toutes les graines germées prêtes à être repiquées sur les sachets.

Au centre du sachet, faire un trou de 2 à $3 \mathrm{~cm}$ de profondeur avec l'index (figure 4.3).

Orienter la graine, tigelle vers le haut (figure 4.4).

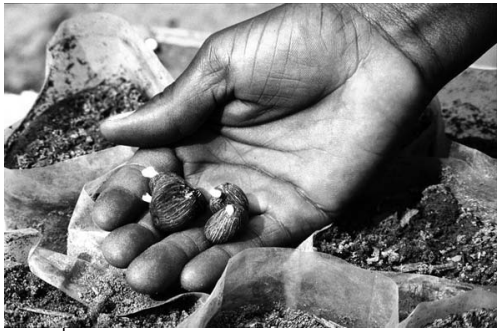

Figure 4.1.

Graines germées

non différenciées.

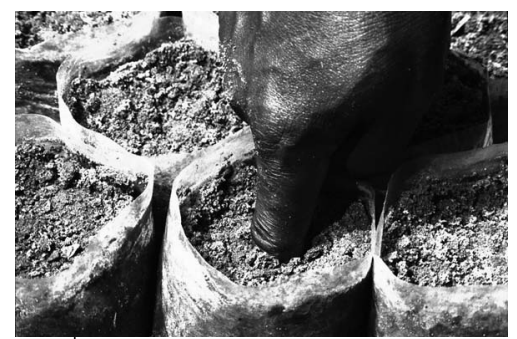

Figure 4.3.

Préparation du trou

de repiquage.

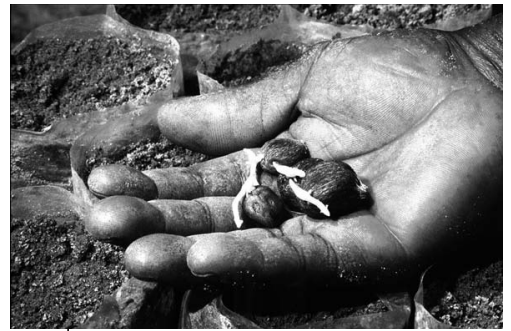

Figure 4.2.

Graines germées

différenciées

prêtes à être repiquées.

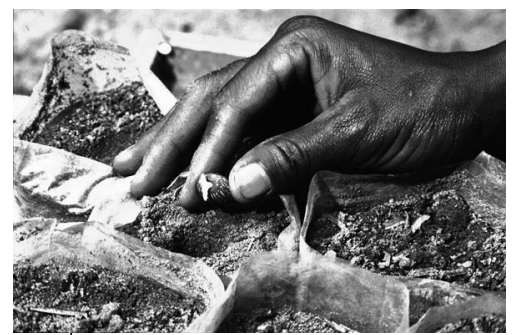

Figure 4.4.

Orientation

de la graine germée. 
Déposer la graine dans le trou (figure 4.5).

Ébouler doucement un peu de terre dans le trou. Il doit y avoir environ $1 \mathrm{~cm}$ de terre au-dessus de la graine (figure 4.6).

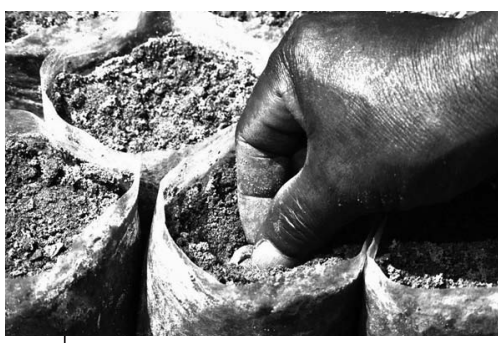

Figure 4.5.

Mise de la graine germée en terre.

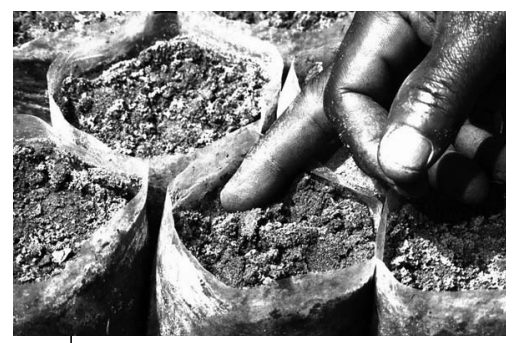

Figure 4.6.

Rebouchage du trou.

Se rincer les mains régulièrement pour faciliter la manipulation des graines germées.

Il ne faut pas placer la tigelle vers le bas ou de travers, ni repiquer la graine trop profondément (mort par pourriture) ou trop en surface (mort par dessèchement). 


\section{Fiche 5. Entretien de la prépépinière}

\section{ID Matériel nécessaire}

- 1 daba ou 1 sarcloir

- 1 râteau

- 1 arrosoir avec pomme.

\section{II) Entretien}

\section{Une fois par semaine}

- arracher, à la main, toutes les repousses de mauvaises herbes dans les sachets (figure 5.1);

- vérifier l'état de la clôture et remplacer éventuellement les éléments défectueux.

\section{Toutes les 2 semaines}

- arracher, à la daba ou au sarcloir, toutes les repousses de mauvaises herbes dans les allées et sur le pourtour de la prépépinière (figure 5.2); - ramasser tous les débris au râteau (figure 5.3);

- jeter les débris hors du périmètre dans une zone à déchets verts réservée à cet effet (figure 5.4).

\section{Arrosage}

S'il a plu suffisamment dans la nuit ou la veille (sol mouillé uniformément et évidence de ruissellement) : pas d'arrosage.

Sinon, apporter un arrosoir plein pour chaque demi-planche soit au total 20 litres d'eau. La pomme de l'arrosoir doit bien répartir l'eau apportée (figure 5.5).

Recommencer tous les 2 jours.

\section{Fumure}

La fumure n'est pas nécessaire à ce stade.

ID Désombrage : uniquement dans le cas d'une pépinière sans ombrière

Commencer lorsque les plantules ont 3 feuilles.

Enlever 1 palme sur 2.

Après 15 jours, enlever encore 1 palme sur 2 (figure 5.6). 
Après 15 jours encore, enlever le reste des palmes.

Les palmes enlevées sont jetées hors du périmètre de la prépépinière et stockées dans la zone de déchets verts réservée à cet effet. Les plantules sont prêtes à être repiquées en pépinière.

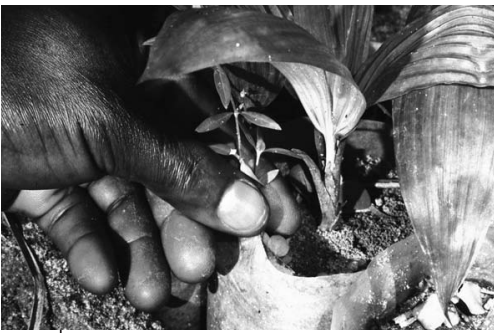

Figure 5.1.

Désherbage manuel des sachets.

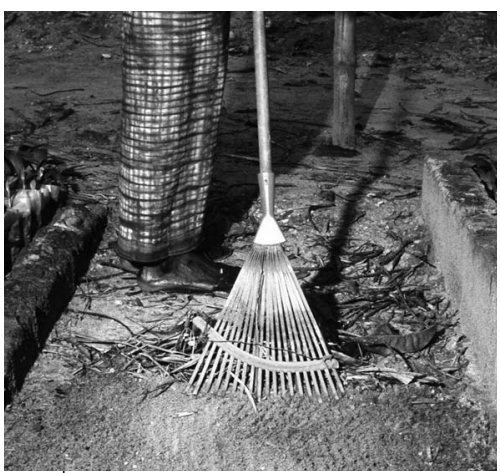

Figure 5.3.

Balayage des allées.

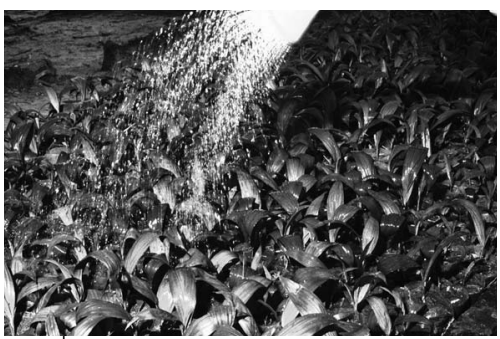

Figure 5.5.

Arrosage des plantules.

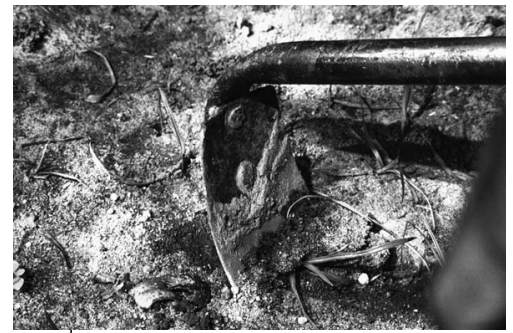

Figure 5.2.

Sarclage des allées.

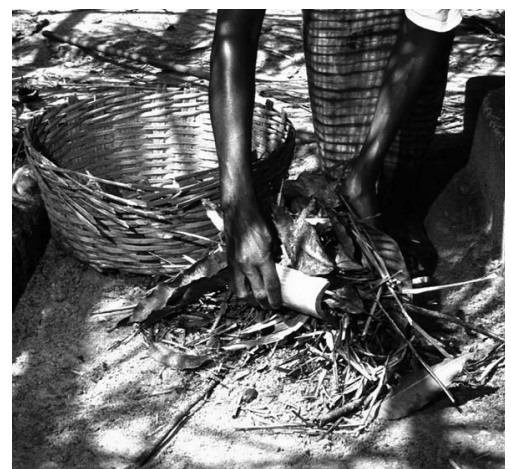

Figure 5.4.

Collecte des déchets verts.

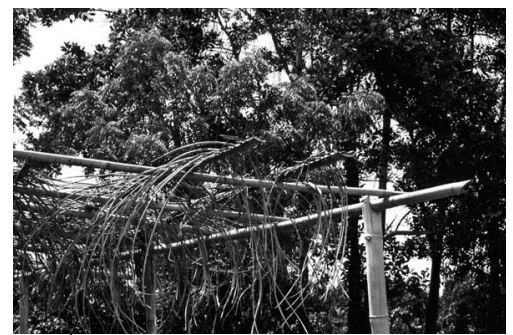

Figure 5.6.

Prépépinière en cours

de désombrage. 


\section{Fiche 6. Problèmes rencontrés dans la prépépinière}

\section{ID Matériel et produits nécessaires}

- Des bouteilles d'eau en plastique

- Un peu de bière

- De la poudre calcaire très sèche

- Produit antilimaces en granulés

- Produit antifourmis en poudre.

Attention, ne pas laisser ces produits à la portée des enfants. Respecter les conseils de protection du fabriquant.

\section{ID Les graines germées d'un même lot} lèvent irrégulièrement

Le terreau est de mauvaise qualité : par exemple, il est trop argileux ou trop sableux.

Le terreau n'a pas été désinfecté.

Les graines n'ont pas été repiquées en temps voulu ou elles ont été mal repiquées.

Larrosage a été trop abondant : les graines sont pourries. Larrosage a été insuffisant : le terreau dans les sachets est dur.

ID Les graines germées sont extraites du sol et des traces de grattage sont visibles sur la surface des sachets

Ce type de dégât peut être causé par des volailles. Il faut vérifier la clôture et faire en sorte que des volailles ne pénètrent pas dans l'enceinte de la prépépinière.

\section{IID Les feuilles sont jaunâtres}

Lombrage est trop clair.

Larrosage est trop abondant.

\section{ID Le bout des feuilles semble brûlé}

Ce symptôme est provoqué par l'anthracnose, maladie fongique induite par un ombrage trop important. 
ID En fin de prépépinière, la partie des feuilles tournée vers le haut est brûlée

Le désombrage a été trop rapide, les feuilles ont été brûlées par le soleil (figure 6.1, voir cahier couleur page 120).

\section{Il y a des limaces ou des escargots dans la planche}

Disposer des pièges autour de la prépépinière : une bouteille d'eau en plastique coupée en deux, enterrée au ras du sol et remplie d'un peu de bière. En cas d'invasion grave, disposer un peu de métaldéhyde en petits tas régulièrement autour de la planche : une dizaine de granulés en petits tas tous les mètres (figure 6.2).

\section{ID Il y a des fourmis ou des termites}

Vérifier s'il n'y a pas de nids cachés dans les bambous de la structure ou du bord des planches.

Détruire ces nids.

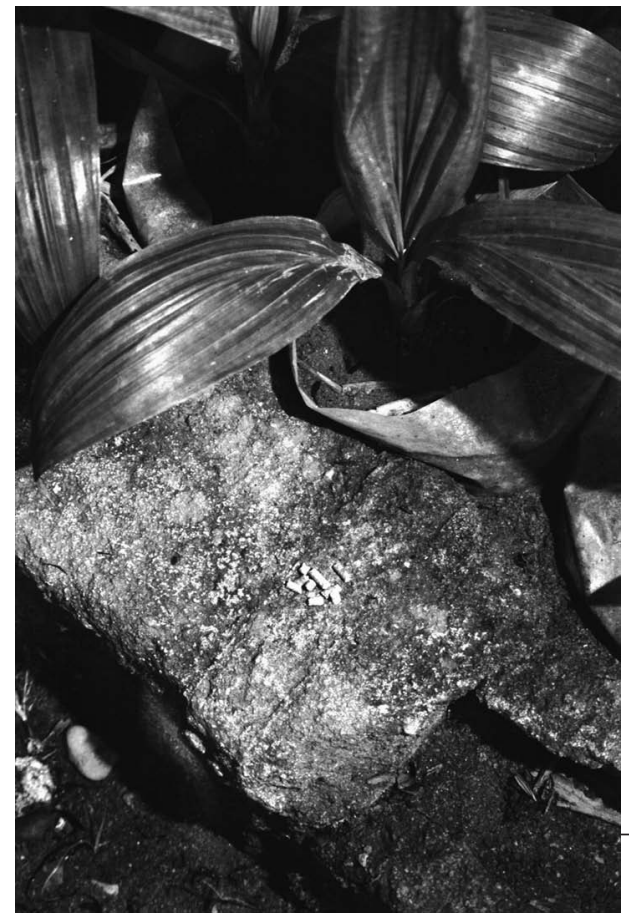

Figure 6.2.

Protection antilimaces.

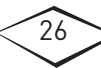


Sinon, disposer un petit cordon de poudre de calcaire très sec autour du grillage de protection (figure 6.3). À défaut, il est possible d'utiliser de la deltaméthrine.

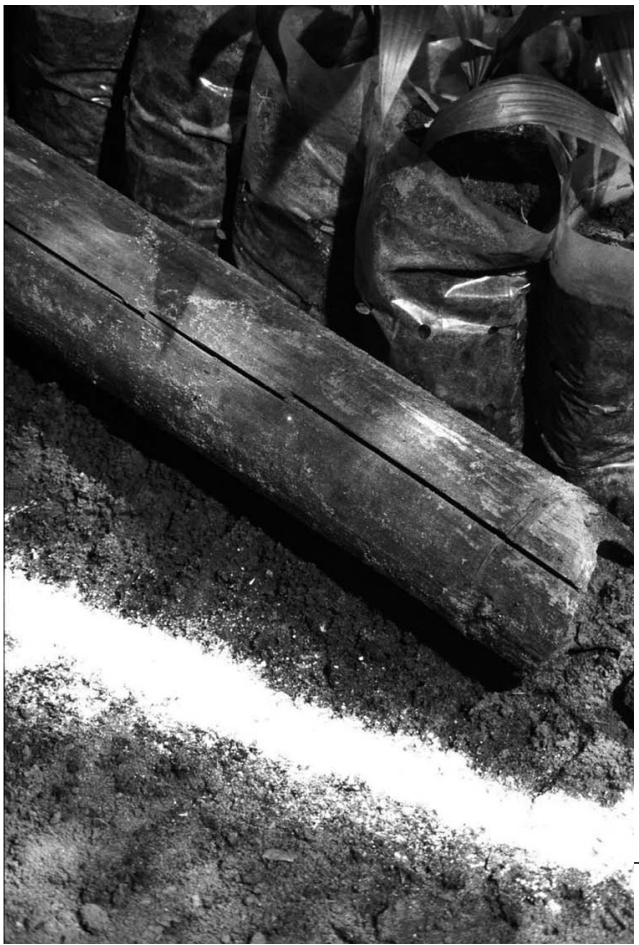

Figure 6.3.

Protection antifourmis.

ID Les plantules sont coupées à ras de façon presque régulière, des traces de sabots sont visibles à la surface des sachets

Il s'agit souvent de dégâts provoqués par du petit bétail, en particulier les chèvres ou les moutons. Il faut vérifier la clôture et faire en sorte que le petit bétail soit éloigné de l'enceinte de la prépépinière.

En cas de nécessité faire appel au conseiller agricole.

\section{Attention}

Ne pas laisser divaguer les volailles ou le petit bétail dans l'enceinte de la prépépinière. Ces animaux peuvent provoquer des dégâts importants aux graines fraîchement repiquées ou aux plantules. 



\section{Pépinière}

La pépinière est la seconde étape de la culture conduisant aux plants de palmier prêts à être plantés. Elle dure de 7 à 9 mois. Pendant ce temps, le palmier perd son aspect juvénile et de véritables palmes commencent à apparaître.

La maladie du blast en pépinière est provoquée par un insecte piqueur (Recilia mica). Cet insecte ne se déplace pas sous un couvert végétal. C'est la raison pour laquelle, à l'origine, les pépinières étaient conduites sous ombrage. Dans les plantations industrielles, la pépinière est conduite sans ombrage depuis que 1'a maladie a pu être contrôlée avec un insecticide systémique :
l'aldicarbe. Néanmoins, cet insecticide, très toxique, n'est pas en vente libre et il n'est accessible sous conditions qu'aux planteurs industriels. Le risque de blast dans les pépinières sans ombrage non traitées est important : de 15 à $60 \%$ de mortalité.

Ce guide propose une conduite de pépinière en deux phases :

- Phase I : dispositif serré sous ombrière;

- Phase II : dispositif normal sans ombrage. 


\section{Fiche 7. Choix et préparation de l'emplacement}

\section{ID Matériel et produits nécessaires}

- 1 machette lourde

- 1 houe ou 1 daba

- Bambous pour poteaux, traverses et piquets de délimitation

- Huile de vidange (5 à 6 litres), sulfate de cuivre ou Xylophène ${ }^{\circledR}$ antitermites sol

- 1 pinceau plat de $5 \mathrm{~cm}$ de large ou équivalent.

\section{ID Choix de l'emplacement}

La pépinière doit être le plus près possible du lieu de plantation.

Elle doit être située à proximité d'un point d'eau abondant, notamment en saison sèche, car la pépinière doit être régulièrement arrosée.

Le terrain doit être bien dégagé, bien drainé et posséder une légère pente pour évacuer les eaux de pluie.

\section{ID Préparation de l'emplacement (figure 7.1)}

Délimiter et désherber totalement un rectangle de 16 x $11 \mathrm{~m}$.

Sur la largeur, tracer une ligne de base à $1 \mathrm{~m}$ de chaque extrémité.

Délimiter 6 planches de 1,5 m de large séparées par des allées d'1 m de large.

Couper 28 gros bambous de 2,5 $\mathrm{m}$ de long.

Entailler une extrémité en « $V$ » et traiter l'autre sur $50 \mathrm{~cm}$ avec de l'huile de vidange, du sulfate de cuivre (à $10 \mathrm{~g} /$ litre) ou du Xylophène ${ }^{\circledR}$ antitermites du sol. Creuser 28 trous de $50 \mathrm{~cm}$ de profondeur le long des planches.

Enfoncer les poteaux de bambous, $\mathrm{V}$ en haut.

Placer 2 traverses en bambous plus légers sur les poteaux, parallèlement aux planches.

Désherber totalement une bande de $10 \mathrm{~m}$ de large tout autour du site de la pépinière.

Si possible, installer une plante légumineuse de couverture, par exemple Pueraria phaseolides. 


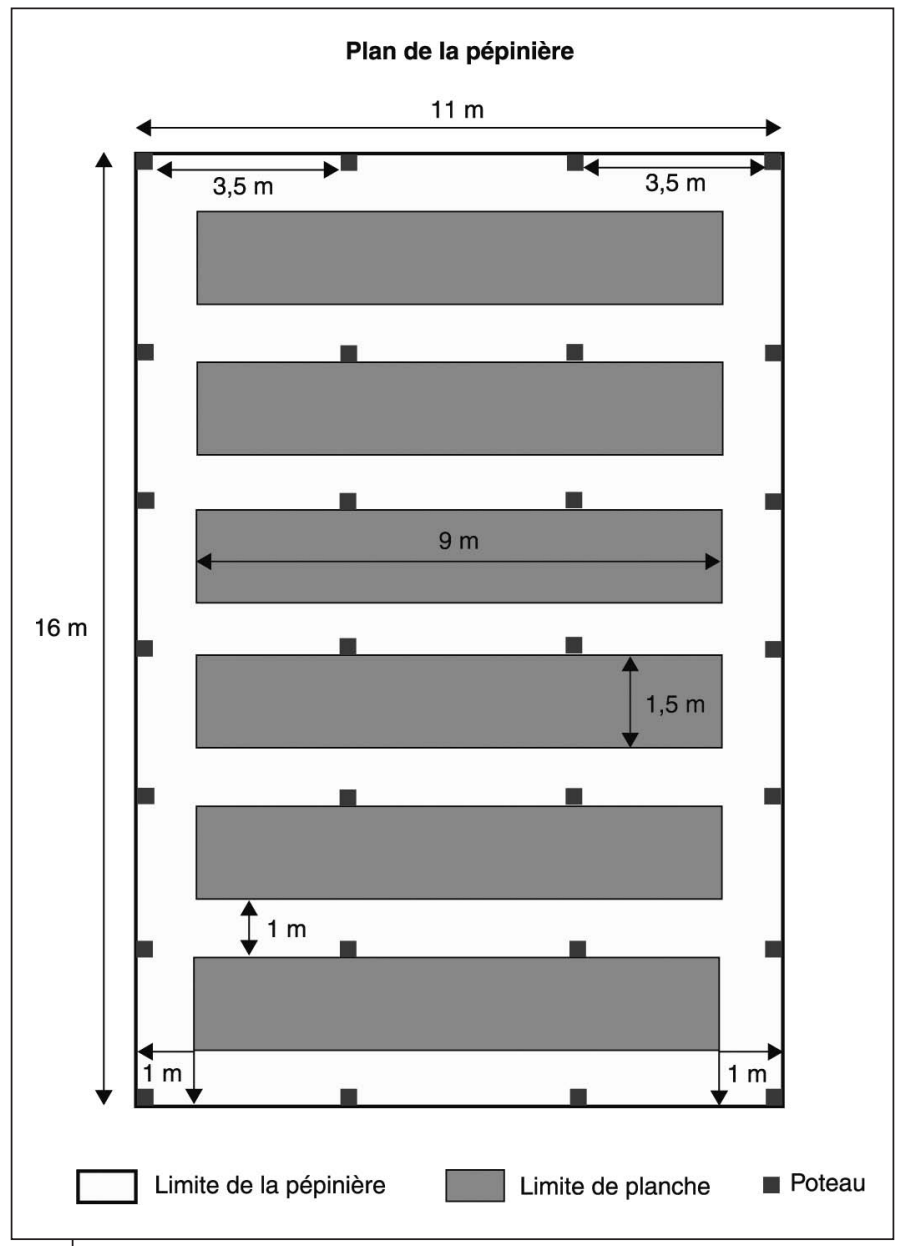

Figure 7.1.

Préparation de l'emplacement. 


\section{Fiche 8. Choix du terreau et remplissage des sacs}

\section{ID Matériel nécessaire}

- Pelles, tines, bâche en plastique, brouette, tamis à mailles de 1 à $2 \mathrm{~cm}$

- 850 sacs noirs

- 17 tonnes de terreau ou de mélange de terreau.

\section{ID Choix du terreau}

Prendre du terreau sous forêt dégradée prélevé dans les 20 premiers centimètres de sol, de préférence léger, humifère et sain.

Passer le terreau dans un tamis à mailles de 1 à $2 \mathrm{~cm}$ (figure 2.1).

Si cela est possible :

- tamiser des déchets domestiques et agricoles bien décomposés pour obtenir du compost;

- mélanger $1 / 3$ de compost ( 1 tine) et $2 / 3$ de terreau ( 2 tines) à la pelle de terrassier;

- protéger le mélange ou le terreau avec une bâche en plastique noir.

\section{Les caractéristiques des sacs à vide}

- Matière : polyéthylène noir sans soufflets

- Épaisseur : 0,15 ou 0,2 mm

- Largeur : $40 \mathrm{~cm}$

- Hauteur : $40 \mathrm{~cm}$

- Base perforée de 3 rangées parallèles de trous espacés de $5 \mathrm{~cm}$.

- Pour 5 ha de plantation $=850$ sacs.

\section{IID Le remplissage des sacs}

(figures 8.1 à 8.3 )

Remplir les sacs jusqu'à $2 \mathrm{~cm}$ du bord supérieur en tassant modérément la terre.

$1 \mathrm{sac}$ plein $=20 \mathrm{~kg}$ de terre $(2$ tines $=3$ sacs $)$.

$850 \mathrm{sacs}=17000 \mathrm{~kg}$ de terreau $=570$ tines environ. 

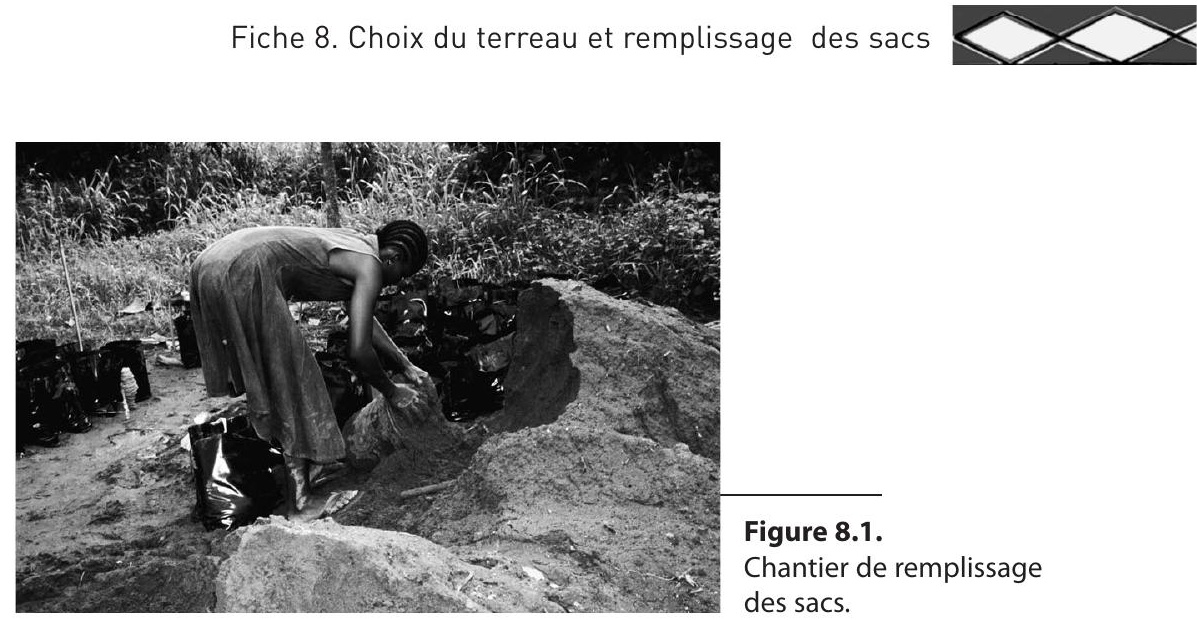

Figure 8.1.

Chantier de remplissage des sacs.

Figure 8.2.

Transfert des sacs vers

le site de la pépinière.
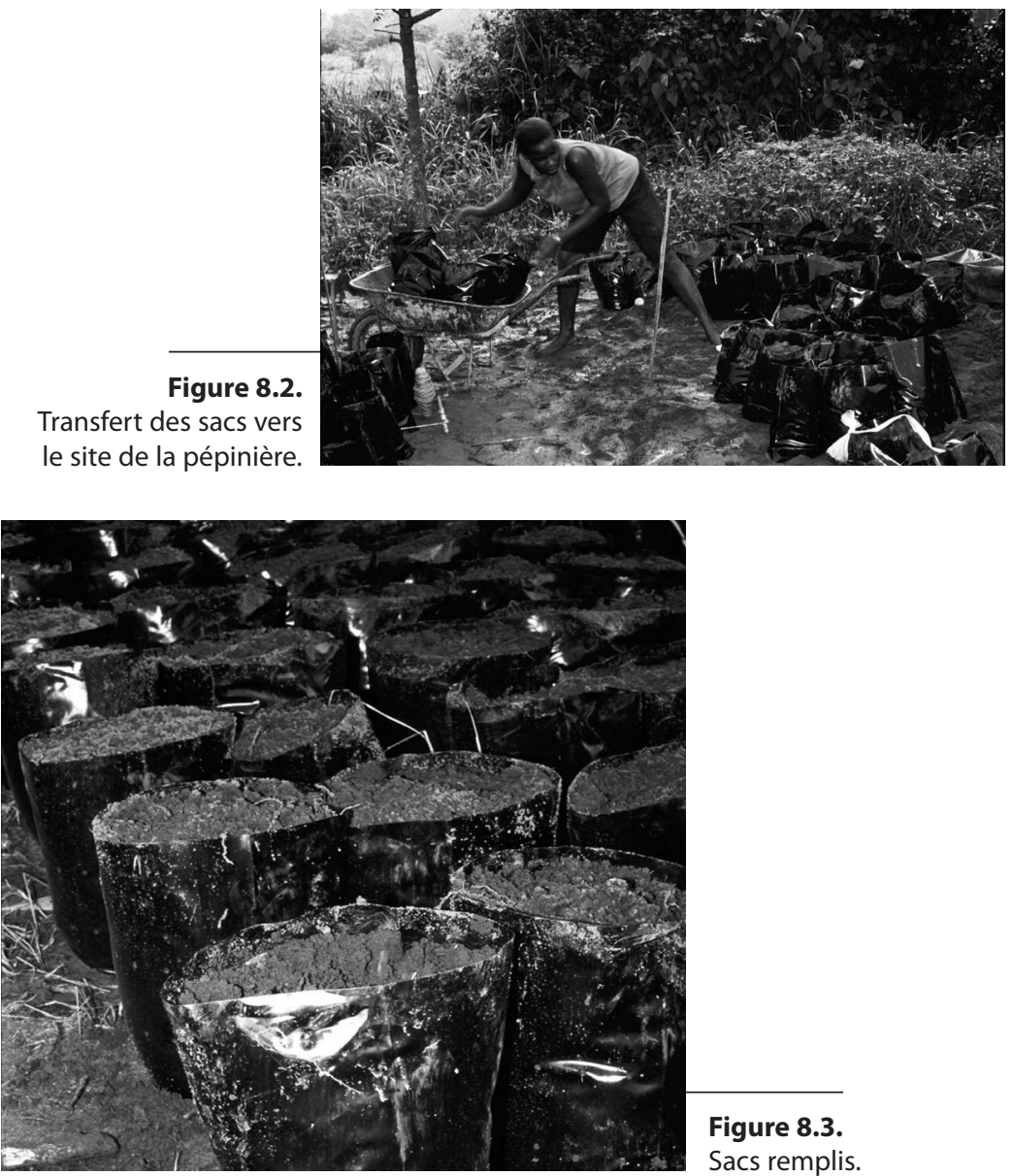

Figure 8.3.

Sacs remplis. 


\section{Fiche 9. Mise en place des sacs et de l'ombrière}

ID Matériel et produits nécessaires

- 1 brouette

- 20 litres d'eau de Javel à $2,6 \%$ ou $20 \mathrm{~g}$ de sulfate neutre d'oxyquinoléine

- $4 \mathrm{~g}$ de deltaméthrine

- 1 arrosoir de 10 litres avec pomme

- 340 feuilles de palmier ou de raphia à changer après 2 mois

- De l'eau claire : 1000 litres environ.

\section{ID Mise en place des sacs}

Le plus tôt possible après le remplissage des sacs.

- dans chaque planche, tracer au cordeau 5 lignes espacées de $30 \mathrm{~cm}$;

- installer les sacs (30 par ligne) en les juxtaposant sur les lignes de façon à ce que les coins soient orientés dans le sens des lignes (figure 9.1).

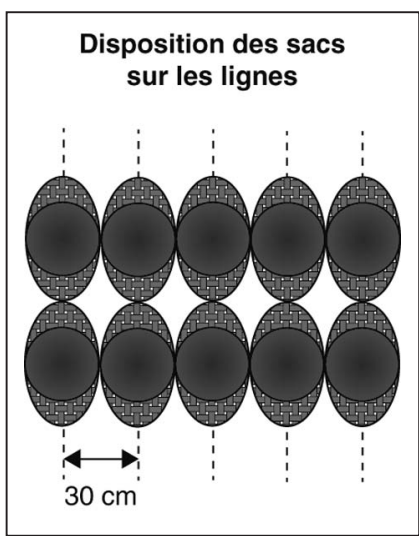

Figure 9.1.

Disposition des sacs sur les lignes.

Traiter le terreau :

- soit avec une solution contenant 1 litre d'eau de Javel à 2,6\% dans 10 litres d'eau;

- soit avec une solution contenant $1 \mathrm{~g}$ de sulfate neutre d'oxyquinoléine à $0,1 \%$ et $0,2 \mathrm{~g}$ de deltaméthrine dans 10 litres d'eau; 


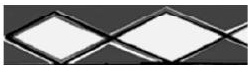

- pour 850 sacs $=20$ arrosoirs environ;

- arroser abondamment pour bien mouiller le terreau jusqu'au fond du sac.

\section{Mise en place de l'ombrière}

Poser les feuilles de palmier ou de raphia, sans leur pétiole, perpendiculairement aux traverses.

Compter 2 à 3 feuilles par mètre courant de traverse soit environ 340 feuilles (figure 9.2).

Changer les feuilles après 2 mois.

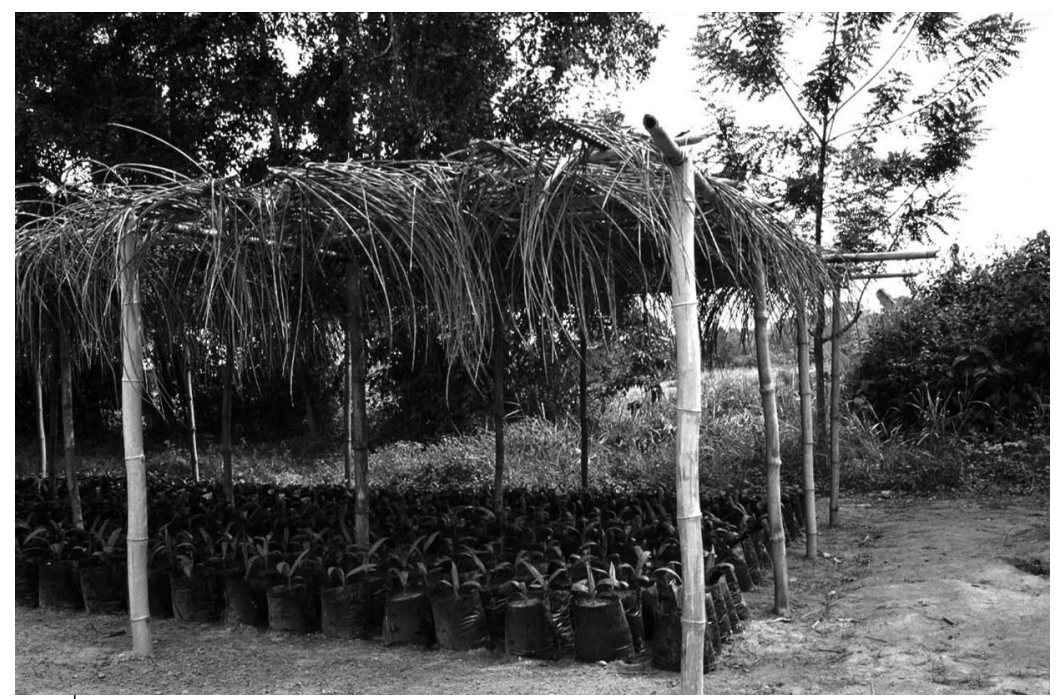

Figure 9.2.

Ombrière en place. 


\section{Fiche 10. Sélection des plantules}

IID Caractéristiques d'une plantule normale en fin de prépépinière

Elle possède 3 à 4 feuilles lancéolées. Chaque feuille émise est, à la fin de sa croissance, plus longue que la précédente. La hauteur, feuille étirée, est de 20 à $25 \mathrm{~cm}$ et la circonférence au collet de $4 \mathrm{~cm}$ au minimum (figure 10.1).

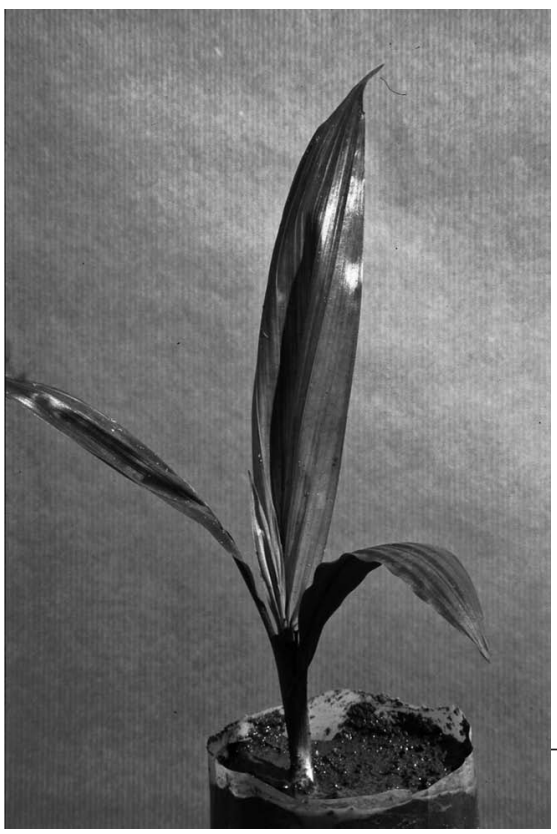

Figure 10.1.

Plantule normale.

\section{IID Caractéristiques des plantules anormales}

Les problèmes suivants peuvent survenir :

- des feuilles enroulées (figure 10.2);

- un port ramassé (figure 10.3);

- des feuilles étroites (figure 10.4);

- la mauvaise ouverture de la nouvelle feuille ou «collante» (figure 10.5);

- une forte attaque d'insectes (figure 10.6).

Toutes les plantules anormales doivent être détruites après le tri. 
IID Pertes normales en prépépinière pour une plantation de 5 ha

Graines non reprises et plantules mortes : $5 \%$ maximum soit 50 graines non reprises ou plantules mortes.

Plantules anormales : $10 \%$ maximum soit 100 plantules.

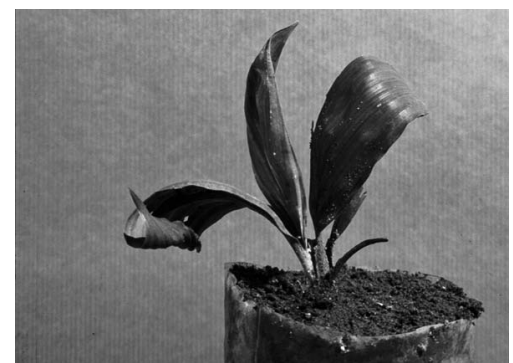

Figure 10.2.

Plantule à feuilles enroulées.

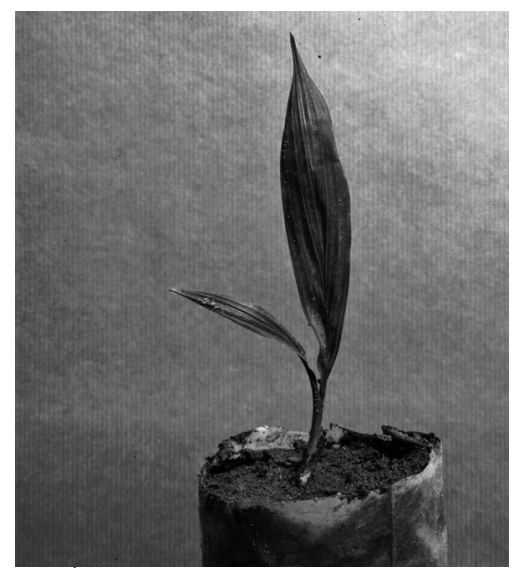

Figure 10.4.

Plantules à feuilles étroites.

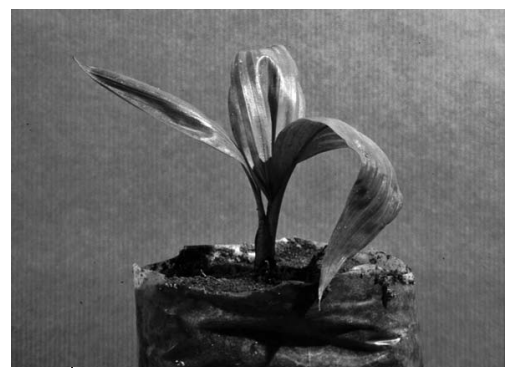

Figure 10.3.

Plantule à port ramassé.

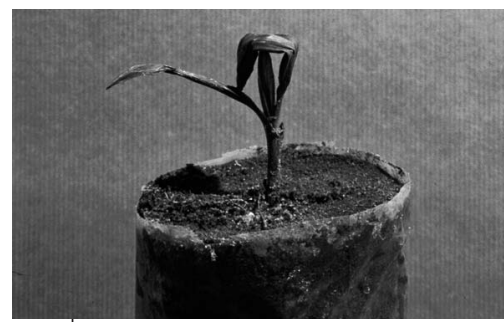

Figure 10.5 .

Plantule souffrant de «collante» : la flèche sort mais la feuille ne se déploie pas.

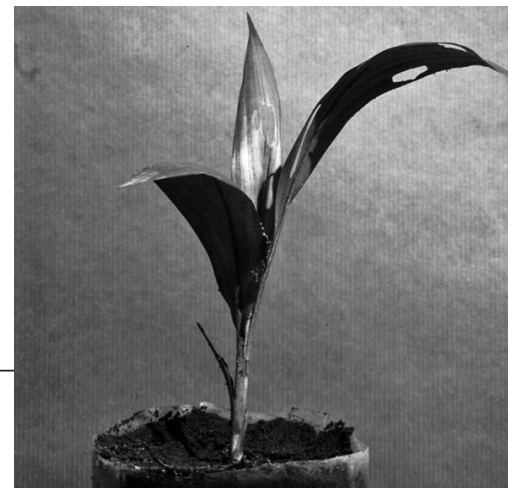




\section{Fiche 11. Transport et repiquage des plantules en pépinière}

Si les plantules ne proviennent pas de la prépépinière conduite par le planteur lui-même, il faut veiller à s'approvisionner chez un producteur de matériel végétal agréé qui garantisse la légitimité et le potentiel de production du matériel végétal. L'utilisation de matériel tout-venant est impropre à la réalisation d'une plantation de qualité en raison de la présence de nombreux anormaux, de palmiers illégitimes et de palmiers consanguins.

\section{Transport des plantules}

Avant le transport, arroser les plantules pour que les mottes soient humides sans excès.

Ranger soigneusement les plantules dans leurs sachets soit :

- dans de grandes bassines pour un transport de quelques mètres (figure 11.1);

- dans une brouette pour un transport de quelques dizaines de mètres (figure 11.2);

- dans une charrette à bras pour un transport de quelques centaines de mètres (figure 11.3);

- au-delà de cette distance, prévoir un transport intermédiaire avec un véhicule de type pick-up.

\section{ID Repiquage des plantules}

Il doit être réalisé le jour même du transport. La veille du repiquage, arroser légèrement le terreau des sacs.

Creuser un trou au centre du sac de la dimension du sachet de prépépinière (figure 11.4).

Déchirer la base du sachet de prépépinière (figure 11.5).

Faire glisser la plantule dans le fond du trou en tirant le sachet de prépépinière vers le haut (figure 11.6).

Combler le trou autour de la motte avec du terreau (figure 11.7).

Tasser normalement le terreau autour de la motte et du collet de la plantule (figure 11.8).

Enlever délicatement toute plantule surnuméraire. Il peut y avoir deux voire trois plantules qui se développent à partir de la graine germée, s'il y a deux ou trois germes qui sortent (figure 11.9). 


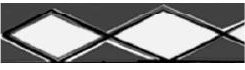

Vérifier que le collet est bien au niveau du sol (figures 11.10 à 11.12). Disposer un paillage de feuilles de légumineuses (par exemple Pueraria phaseolides) grossièrement hachées sur toute la surface du sol du sac. Si nécessaire, repiquer les plantules normales surnuméraires à racines nues.

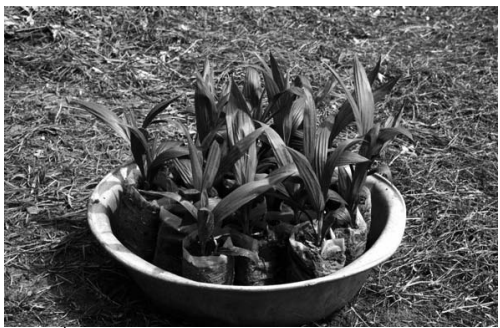

Figure 11.1.

Transport des plantules en bassine.

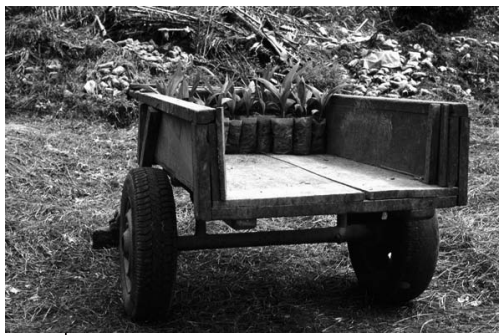

Figure 11.3.

Transport des plantules en charrette à bras.

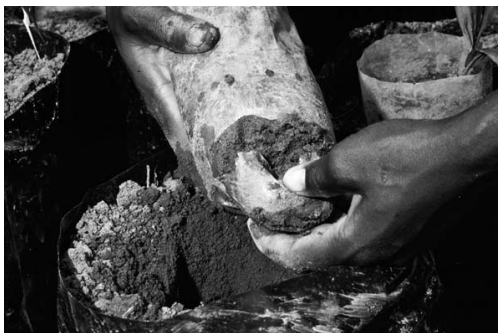

Figure 11.5.

Déchirer le fond du sac.

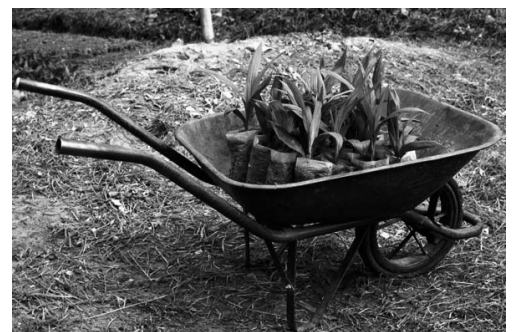

Figure 11.2.

Transport des plantules en brouette.

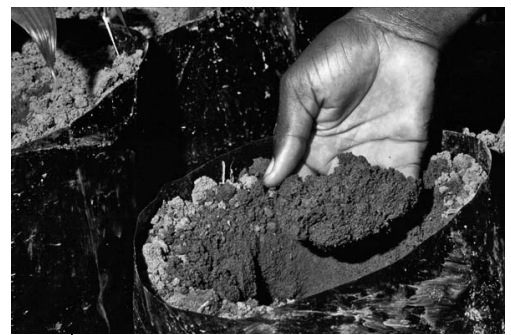

Figure 11.4.

Préparation du trou

dans le terreau du sac.

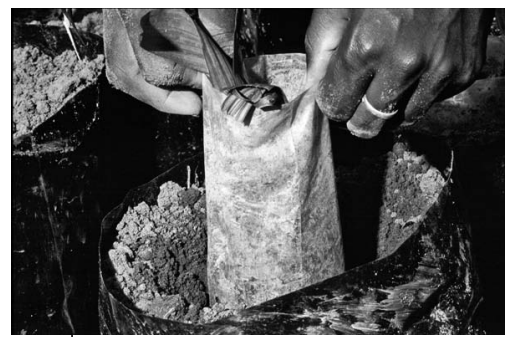

Figure 11.6.

Faire glisser le sachet vers le haut. 


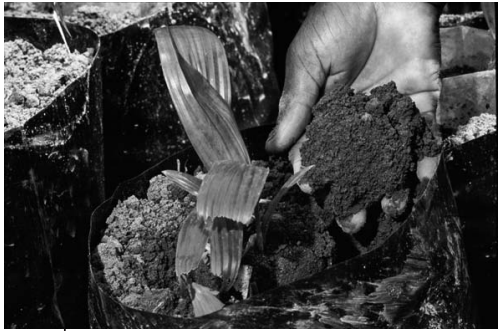

Figure 11.7.

Combler le trou autour de

la motte avec du terreau.

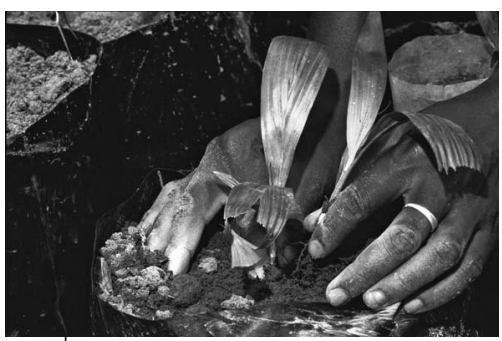

Figure 11.9.

Enlever délicatement

les plantules surnuméraires.

Figure 11.10.

Plantule bien repiquée.

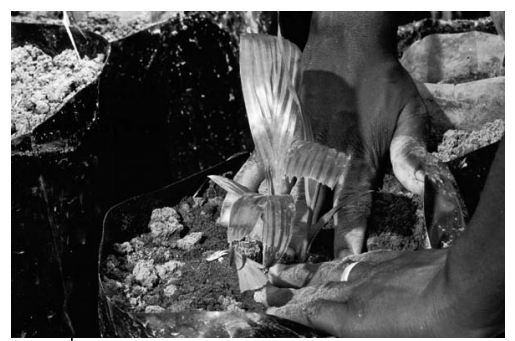

Figure 11.8.

Tasser modérément le terreau autour du collet.
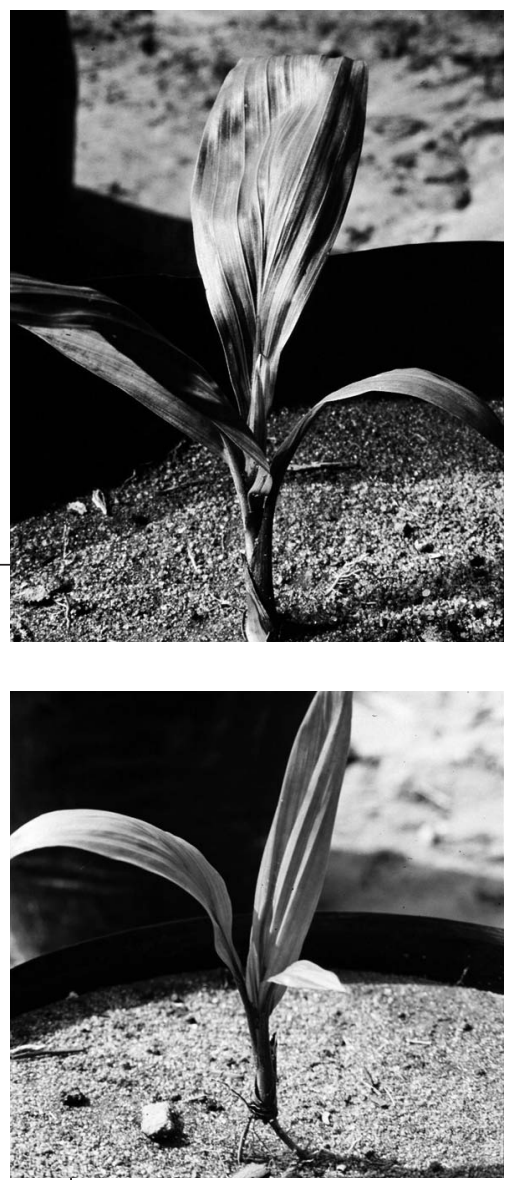

Figure 11.12.

Plantule déchaussée.
Figure 11.11.

Collet trop enterré.

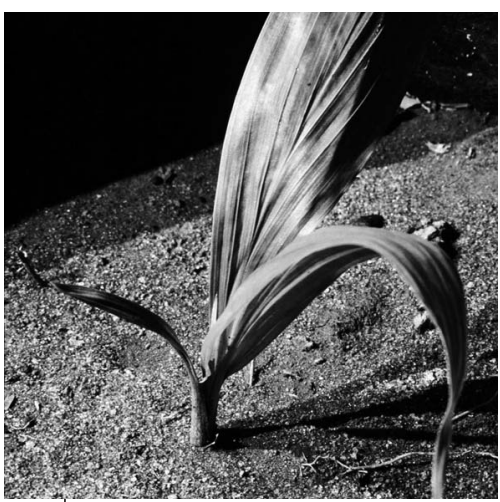




\section{Fiche 12. Entretien de la pépinière}

\section{Matériel et produits nécessaires}

- 1 daba ou 1 sarcloir, 1 râteau, 1 panier

- 1 pulvérisateur à pression préalable de 5 litres marqué $\mathrm{F}$ pour fongicide

- 1 récipient cylindrique de 0,5 litre de capacité

- 1 récipient cylindrique de 1 litre de capacité

- 1 bouchon de bouteille d'eau ou une capsule de bouteille de bière

- 1 éclat de bambou pour l'enfouissement de l'engrais

- Du paillage déjà préparé

- 5 fûts de 200 litres bien lavés pour stocker l'eau

- 1 seau de 5 litres pour préparer les bouillies

- Fongicide par paquet de $1 \mathrm{~kg}$ à renouveler.

\section{Sarclage}

Une fois par mois ou selon l'état d'envahissement de la pépinière, il faut arracher :

- à la main, les mauvaises herbes dans les sacs (figure 12.1);

- à la daba ou au sarcloir, toutes les mauvaises herbes poussant entre les sacs et sur tout le périmètre de la pépinière (figure 12.2).

Ramasser tous les débris au râteau (figures 12.3 et 12.4).

Les jeter hors du périmètre sur le site de collecte des déchets verts.

\section{ID Traitement fongicide}

En Afrique, les plants sont pratiquement toujours attaqués par la cercosporiose. C'est le seul cas où un traitement préventif est recommandé, car si cette maladie foliaire n'est pas contrôlée, elle limite fortement la croissance des plantes. Pour lutter contre la cercosporiose il faut traiter tous les 15 jours à partir de l'apparition des premiers symptômes foliaires, le dessous et le dessus des feuilles (figures 12.5 et 12.6) avec une bouillie préparée avec l'un des produits présentés au tableau 12.1. (Voir aussi Fiche 41). Les quantités à apporter par tour de traitement et pour 850 plants sont indiquées dans le tableau 12.2. 
Tableau 12.1. Fongicides recommandés :

matière active et concentration de produit calculée par litre d'eau.

\begin{tabular}{lc}
\hline Matière active & Concentration (g / litre de bouillie) \\
\hline Bénomyl & 0,5 \\
\hline Chlorothalonil & 1,5 \\
\hline Manèbe & 2,0 \\
\hline Mancozèbe & 2,0 \\
\hline Thiophanate-méthyl & 1,0 \\
\hline
\end{tabular}

Tableau 12.2. Matériel et quantités requises en fonction de l'âge des plants.

\begin{tabular}{lcccccccc}
\hline Caractéristiques du traitement & \multicolumn{8}{c}{$\hat{\text { Âge des plants en pépinière (en mois) }}$} \\
\cline { 2 - 9 } & $\mathbf{1}$ & $\mathbf{2}$ & $\mathbf{3}$ & $\mathbf{4}$ & $\mathbf{5}$ & $\mathbf{6}$ & $\mathbf{7}$ & $\mathbf{8}$ \\
\hline $\begin{array}{l}\text { Nombre de remplissages } \\
\text { du pulvérisateur }\end{array}$ & 3 & 6 & 9 & 13 & 16 & 20 & 23 & 25 \\
\hline Volume d'eau nécessaire (litres) & 15 & 30 & 45 & 65 & 80 & 100 & 115 & 125 \\
\hline $\begin{array}{l}\text { Nombre plants traités } \\
\text { avec la quantité de produit } \\
\text { d'un pulvérisateur }\end{array}$ & 285 & 145 & 95 & 65 & 55 & 45 & 40 & 35 \\
\hline
\end{tabular}

Utiliser un pulvérisateur à pression préalable de 5 litres de capacité n'ayant jamais servi pour pulvériser des herbicides. Utiliser deux des produits cités ci-dessus en alternance toutes les deux semaines.

Il ne faut jamais traiter sous la pluie.

\section{ID Préparation de la bouillie}

\section{La veille du jour de traitement}

Prévoir les quantités d'eau nécessaires.

Vérifier le matériel et le stock des produits fongicides.

Compléter ou réparer si nécessaire.

\section{Le jour du traitement}

Disposer sur une aire sèche le matériel et les produits nécessaires : fongicide, dosette, seau, bambou et pulvérisateur.

Ne pas oublier de s'équiper avec les vêtements de protection.

Préparer avec la dosette fournie la quantité de produit nécessaire pour 1 pulvérisateur.

Remplir un demi-seau de 5 litres de capacité d'eau propre. 


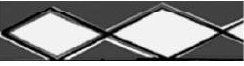

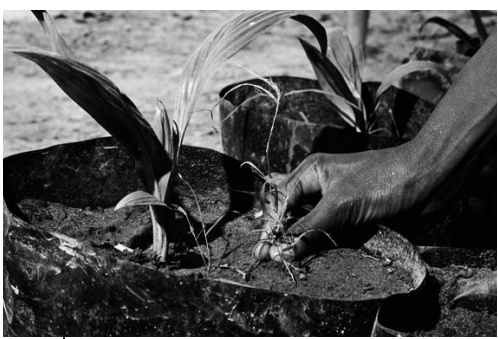

Figure 12.1.

Sarclage dans les sacs.
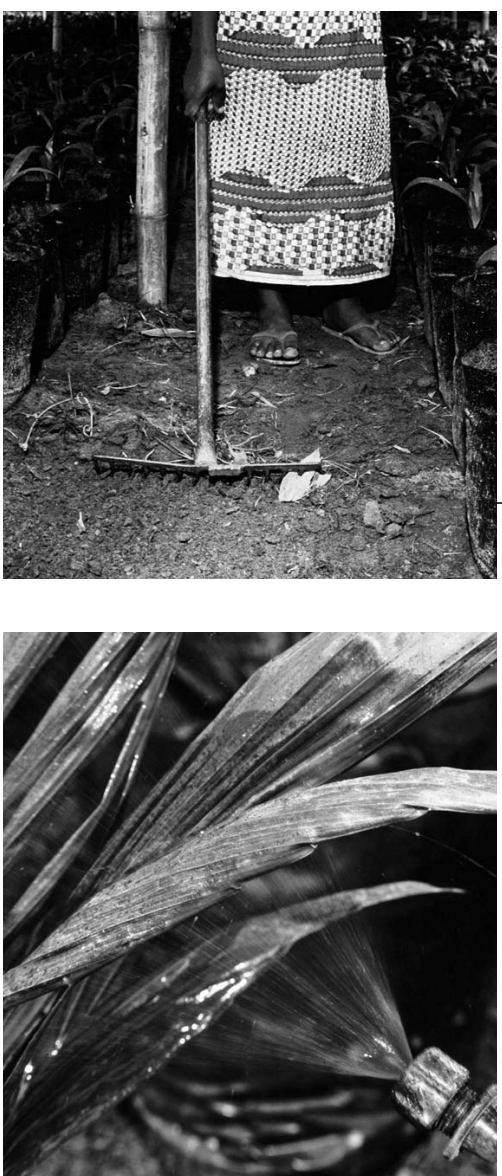

Figure 12.5.

Traitement antifongique

au-dessous des feuilles.

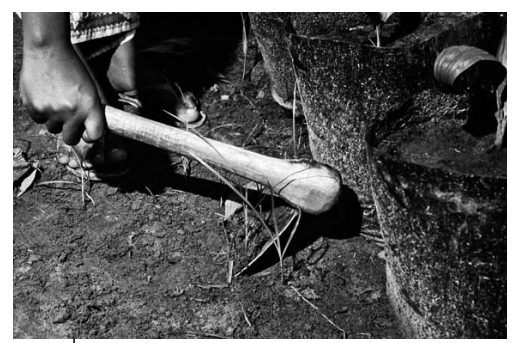

Figure 12.2.

Sarclage entre les sacs.

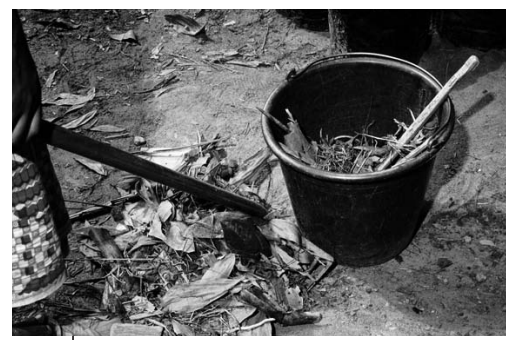

Figure 12.4.

Élimination des débris.

Figure 12.3.

Ramassage des débris.

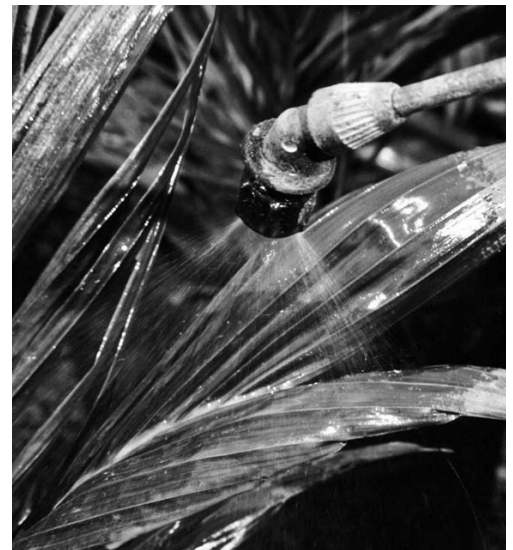

Figure 12.6.

Traitement antifongique au-dessus des feuilles. 
Verser doucement la poudre ou le liquide en agitant doucement le contenu du seau avec un petit bambou pour éviter les grumeaux.

Verser le mélange dans le pulvérisateur à travers un entonnoir équipé d'un filtre.

Compléter avec de l'eau propre jusqu'à 5 litres.

Bien agiter avant de commencer à pulvériser.

\section{Arrosage}

Il doit être effectué 3 jours par semaine : par exemple : lundi, mercredi et vendredi.

Jusqu'à la fin du $4^{\mathrm{e}}$ mois : 0,5 litre d'eau par plant.

À partir de la fin du $4^{\mathrm{e}}$ mois : 1 litre d'eau par plant.

Leau doit être aussi propre que possible et sans risque de pollution.

Disposer si possible 3 à 5 fûts propres de 220 litres de capacité tout autour de la pépinière.

Les remplir d'eau la veille au soir s'il n'y a pas une source d'eau courante à la pépinière.

Utiliser des récipients de forme cylindrique de capacité adéquate $(0,5$ ou 1 litre).

Ne pas jeter l'eau au pied des plantules mais la faire couler doucement au rythme de son absorption afin de ne pas déchausser la plante (figure 12.7).

Il est préférable d'effectuer l'arrosage tôt le matin ou en fin d'après-midi.

\section{ID Fumure}

Le terreau ayant été bien préparé, apporter une fois par mois :

- jusqu'à la fin du $4^{\mathrm{e}}$ mois : $5 \mathrm{~g}$ d'urée par plant;

- à partir de la fin du $4^{\mathrm{e}}$ mois : $10 \mathrm{~g}$ d'urée par plant.

Enlever le paillage.

Bien répartir l'engrais sur toute la surface du sac sous le paillage (figure 12.8).

Enfouir l'engrais par un léger griffage (figure 12.9).

Replacer le paillage ou le changer si nécessaire.

Ne pas oublier d'arroser immédiatement après.

\section{Attention}

1 bouchon de bouteille d'eau plastique ou une capsule métallique de bouteille de bière contient $5 \mathrm{~g}$ d'urée. 


\section{IID Désombrage}

À la fin du $4^{\mathrm{e}}$ mois, enlever 1 palme sur 2 .

Après 15 jours, enlever encore 1 palme sur 2 (voir Fiche 5).

Après 15 jours encore, enlever le reste des palmes.

Les palmes enlevées sont jetées hors du périmètre de la pépinière dans la zone de collecte des déchets verts.

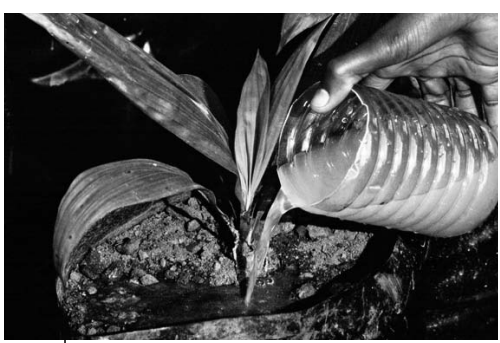

Figure 12.7.

Arrosage des plantes.

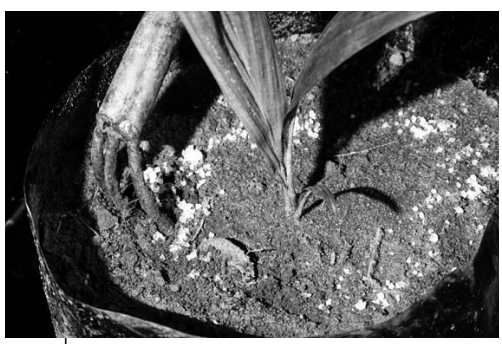

Figure 12.9.

Enfouissement de l'engrais

par griffage du sol.

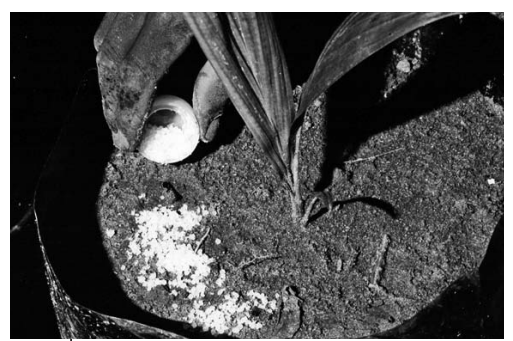

Figure 12.8.

Épandage de l'engrais. 


\section{Fiche 13. Mise à écartement}

\section{ID Préalable}

Enlever tous les supports de l'ombrière encore en place.

Couper 565 piquets en éclats de bambou : longueur $25 \mathrm{~cm}$ environ, largeur $1 \mathrm{~cm}$ environ.

Délimiter et nettoyer complètement une bande de $10 \mathrm{~m}$ de large à chaque extrémité des planches.

\section{ID Piquetage des nouveaux emplacements}

Dans l'alignement des colonnes 1, 3 et 5 de chaque planche, matérialiser un prolongement de $9 \mathrm{~m}$ à chaque extrémité.

Dans le prolongement des colonnes 1 et 5 de chaque planche :

- à l'extrémité 1 , placer le premier piquet à $30 \mathrm{~cm}$ du premier sac et les 14 suivants à $60 \mathrm{~cm}$ les uns des autres;

- à l'extrémité 2, placer le premier piquet à $60 \mathrm{~cm}$ du dernier sac et les 14 suivants à $60 \mathrm{~cm}$ les uns des autres.

Dans le prolongement de la colonne 3 de chaque planche :

- à l'extrémité 1 , placer le premier piquet à $60 \mathrm{~cm}$ du premier sac et les 14 suivants à $60 \mathrm{~cm}$ les uns des autres,

- à l'extrémité 2 , placer le premier piquet à $30 \mathrm{~cm}$ du dernier sac et les 14 suivants à $60 \mathrm{~cm}$ les uns des autres.

Faire de même pour chaque planche :

- au milieu de chaque allée entre planches, matérialiser une colonne;

- placer 1 piquet au même niveau que les premiers sacs de l'extrémité 1 et des premiers sacs de l'extrémité 2 des planches;

- au-delà de l'extrémité 1 de la planche, placer, sur la colonne, 15 piquets distants de $60 \mathrm{~cm}$ les uns des autres;

- vers l'extrémité 2 , placer sur la colonne 29 piquets distants de $60 \mathrm{~cm}$ les uns des autres.

\section{ID Déplacement des sacs}

Lobjectif est de déplacer les sacs tout en maintenant en place un certain nombre d'entre eux.

Commencer par l'extrémité 1 de la première planche (figure 13.1).

Déplacer les 3 sacs du milieu de la première ligne vers les nouveaux emplacements, les deux sacs latéraux restent en place. 
Sur la ligne 2, déplacer vers les nouveaux emplacements les sacs placés à droite et à gauche du sac central qui reste en place.

Sur la ligne 3, puis les lignes d'ordre impair, procéder comme pour la première ligne.

Sur la ligne 4, puis les lignes d'ordre pair, procéder comme pour la ligne 2.

Lorsque tous les nouveaux emplacements au-dessus de l'extrémité 1 ont été pourvus, poursuivre les déplacements vers les emplacements matérialisés au-dessous de l'extrémité 2.

Suivre la même procédure pour les planches suivantes.

Les sacs contenant les plants morts ou très malades sont vidés sur place et les plants jetés dans le lieu de collecte des débris végétaux. Les emplacements concernés seront occupés par des sacs contenant des plants sains.

À la fin des opérations, tous les plants seront disposés en quinconce sur 19 colonnes de 45 sacs environ (figure 13.2).

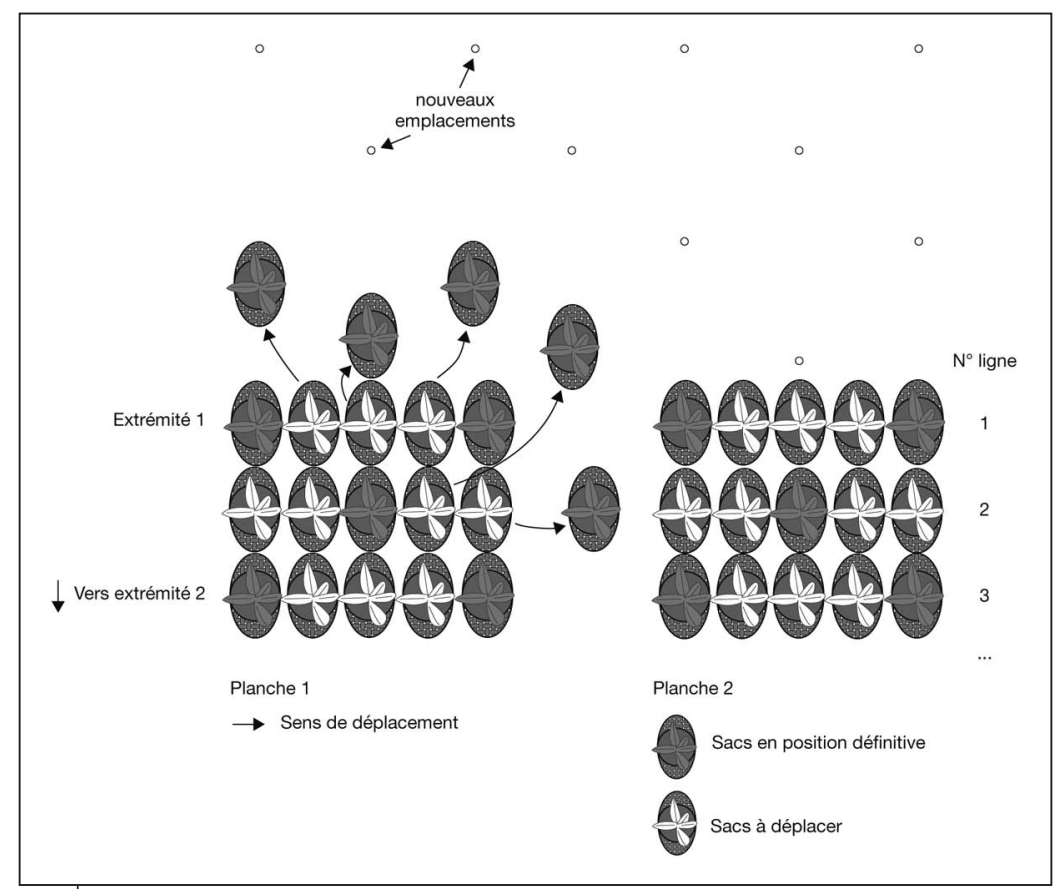

Figure 13.1.

Déplacement des sacs. 


\section{Fiche 14. Sélection des plants}

La sélection des plants doit se faire au cours du $7^{\mathrm{e}}$ mois de la pépinière. Un plant anormal ne produit que très peu ou pas du tout. Donc, une bonne sélection garantit une production normale de tous les arbres plantés (figure 14.1). Laspect des plants est le critère le plus important, la dimension des plants est un critère secondaire.

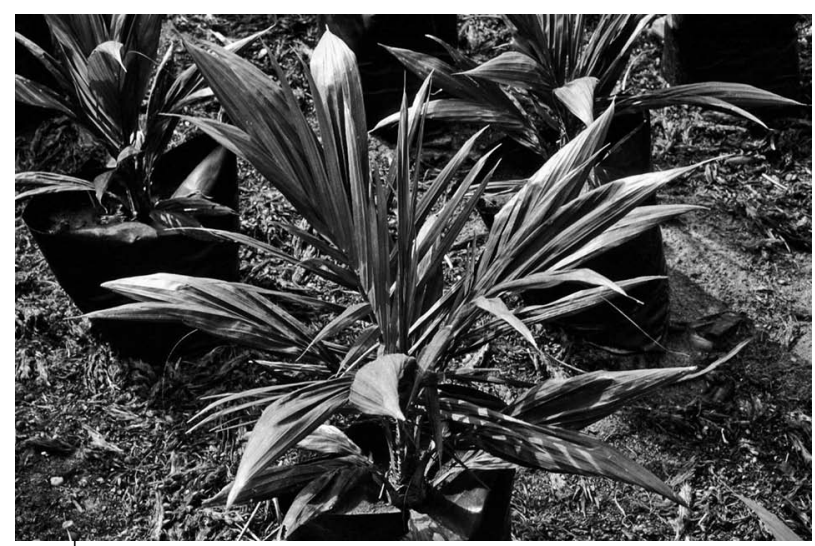

Figure 14.1.

Plant normal.

Éliminer les plants anormaux tels que :

- les plants malades;

- les plants partiellement ou entièrement décolorés;

- les plants très attaqués par les insectes;

- les plants très attaqués par les champignons des feuilles;

- les plants chétifs;

- les plants dont les feuilles sont anormales, par exemple ayant des folioles soudées, courtes, étroites ou insérées à angle aigu (figures 14.2 et 14.3).

Identifier les plants éliminés avec une baguette de bambou de 1,2m plantée dans la terre du sac.

Arracher les plants éliminés et les détruire à la machette.

Jeter ces plants éliminés sur le site de collecte des déchets verts.

Dans une pépinière bien conduite, on doit éliminer au maximum $10 \%$ des plants. Au-delà, la conduite de pépinière n'a pas été faite convenablement. 


\section{Fiche 15. Problèmes rencontrés dans la pépinière}

Consulter aussi le chapitre 7 «Défense des cultures» page 119.

Les figures de ce chapitre sont insérées dans le cahier couleur.

Matériel et produits éventuellement nécessaires

- Diméthoate

- Deltaméthrine

- Un pulvérisateur de 5 litres

- Le matériel pour préparer la solution insecticide, n'ayant jamais servi pour un traitement insecticide

- L'équipement de protection individuel (gants, masque, tablier, bottes)

- De l'eau claire.

\section{Attention}

Ne pas laisser ces produits à la portée des enfants. Respecter les conseils d'usage et de protection du fabriquant.

\section{La flèche du plant est entièrement pourrie} puis le plant se dessèche

Si le problème apparaît entre septembre et mars, il s'agit probablement du blast (voir cahier couleur figure 15.1).

Bien veiller à supprimer toutes les graminées qui sont les hôtes du vecteur de la maladie, dans et autour de la pépinière.

Renforcer l'ombrage.

Détruire tous les plants malades.

ID La flèche du plant ne pousse plus, et présente des petites taches jaunâtres

Il s'agit probablement de la pourriture sèche du cœur (voir cahier couleur figure 15.2).

Bien veiller à supprimer toutes les graminées qui sont aussi les hôtes du vecteur de la maladie, dans et autour de la pépinière.

Renforcer l'ombrage.

Détruire tous les plants malades. 
IID L'extrémité des feuilles basses est brûlée, surtout le long des nervures, la croissance est ralentie

Il s'agit probablement de brûlures d'engrais (voir cahier couleur figure 15.3). L'épandage d'engrais a été fait trop près du collet.

Larrosage est absent ou insuffisant.

Il faut suspendre tout épandage d'engrais jusqu'au rétablissement de la croissance et contrôler la qualité de l'arrosage.

\section{Les feuilles sont envahies de taches orange} avec des ponctuations noires

Les plants sont atteints de la maladie provoquée par le champignon Cercospora elaeidis (voir cahier couleur figure 15.4). Il faut mettre en place impérativement les traitements antifongiques hebdomadaires en alternant les matières actives.

Il faut vérifier aussi les conditions d'arrosage.

\section{ID) La flèche sort mais la feuille ne se déploie pas}

Ce symptôme s'appelle «la collante» (voir cahier couleur figure 15.5). Le plus souvent, il est le signe d'un manque ponctuel d'eau.

\section{Il y a des vers blancs dans la base des feuilles}

Extraire les vers blancs, ce sont des larves de Temnoschoita sp. (voir cahier couleur figure 15.6).

Détruire les plants trop atteints.

Nettoyer les alentours de la pépinière et jeter les débris qui sont potentiellement des refuges dans la zone de collecte des déchets végétaux.

Si le problème persiste, traiter tous les plants avec une solution de diméthoate à $0,8 \mathrm{~g}$ de matière active par litre.

\section{ID Les folioles sont comme décapées par plaques}

Ces symptômes sont le signe de la présence de criquets (criquets verts, criquets puants).

Extirper Chromolaena odorata des abords de la pépinière.

Si le problème persiste, traiter tous les plants et les abords de la pépinière avec une solution de deltaméthrine à $0,02 \mathrm{~g}$ de matière active par litre. 
II Le limbe des feuilles, surtout des plus latérales, est découpé montrant des traces assez larges d'arrachement plus ou moins régulier; des traces de sabots sont visibles à la surface du sol

Il s'agit le plus souvent de dégâts provoqués par du petit ou du gros bétail (chèvres, moutons ou bovins).

Il faut faire en sorte de modifier le parcours de pacage de ces animaux et de renforcer leur surveillance.

En cas de nécessité, faire appel au conseiller agricole.

\section{Attention}

Veillez à ne pas laisser divaguer le petit et le gros bétail dans la pépinière. 



\section{Pépinière directe}

Les planteurs plus expérimentés peuvent supprimer, s'ils le souhaitent, le stade prépépinière et repiquer directement les graines germées dans les grands sacs.

Pour réaliser une pépinière directe, il faut réaliser les travaux repris dans les fiches des chapitres Prépépinière, Pépinière et Pépinière directe, dans l'ordre indiqué dans le tableau 3.

Cette technique permet de gagner 1 à 1,5 mois sur la durée totale du cycle classique.

Tableau 3. Ordre des opérations en pépinière directe.

\begin{tabular}{llc}
\hline Ordre des opérations & Chapitre & $\mathbf{N}^{\circ}$ de Fiche \\
\hline Choix de l'emplacement & Pépinière & 7 \\
\hline Choix du terreau et remplissage des sacs & Pépinière & 8 \\
\hline Mise en place des sacs et de l'ombrière & Pépinière & 9 \\
\hline Tri et repiquage des graines germées & Prépépinière & 4 \\
\hline Entretien de la pépinière directe & Pépinière directe & 16 \\
\hline Mise à écartement & Pépinière & 13 \\
\hline Sélection des plants & Pépinière & 14 \\
\hline Problèmes rencontrés dans la pépinière & Pépinière & 15 \\
\hline
\end{tabular}




\section{Fiche 16. Entretien de la pépinière directe}

\section{ID Matériel nécessaire}

- 1 daba ou 1 sarcloir, 1 râteau, 1 panier

- 1 pulvérisateur à pression préalable de 5 litres marqué $\mathrm{F}$ (pour Fongicide)

- 1 récipient à large ouverture de 0,5 litre

- 1 récipient à large ouverture de 1 litre

- 1 bouchon de bouteille d'eau

- 1 éclat de bambou ou une griffe pour l'enfouissement de l'engrais

- 5 fûts de 200 litres bien lavés pour stocker l'eau

- 1 seau de 5 litres pour préparer les bouillies.

\section{II) Sarclage}

Une fois par mois :

- Arracher les mauvaises herbes à la main dans les sacs.

- Arracher à la daba ou au sarcloir, toutes les mauvaises herbes entre les sacs et sur tout le périmètre de la pépinière.

- Ramasser tous les débris au râteau. Jeter les débris dans les espaces de déchets verts hors du périmètre.

\section{II) Démariage}

À la fin du $4^{\mathrm{e}}$ mois ou lorsque les plantules ont en moyenne 3 feuilles :

- Vérifier qu'il n'y a qu'une plantule par sac.

S'il y a deux plantules ou plus par sac:

- Enlever soigneusement les plantules surnuméraires.

- Éventuellement, repiquer les plantules supplémentaires dans les sacs dont les graines germées n'ont pas levé ou dont les plantules sont mortes.

- Consulter la fiche repiquage des plantules en pépinière (Fiche 11).

\section{ID Traitement fongicide}

Si des signes de cercosporiose apparaissent, traiter tous les 15 jours à partir des premiers symptômes visibles sur les feuilles.

Traiter en se référant à la Fiche 12 : «Entretien de la pépinière». 
ID Fumure

Le terreau ayant été bien préparé, apporter une fois par mois :

- de la fin du $3^{\mathrm{e}}$ mois à la fin du $7^{\mathrm{e}}$ mois : $5 \mathrm{~g}$ d'urée par plant.

- à partir de la fin du 7e mois : $10 \mathrm{~g}$ d'urée par plant.

Enlever le paillage.

Bien répartir l'urée sur toute la surface du sac et l'enfouir par un léger griffage.

Replacer le paillage ou le remplacer si nécessaire.

Arroser immédiatement après.

\section{Attention}

1 bouchon de bouteille d'eau plastique ou une capsule métallique de bouteille de bière contient $5 \mathrm{~g}$ d'urée.

\section{ID Désombrage}

Commencer à la fin du $7^{\mathrm{e}}$ mois.

Enlever 1 palme sur 2.

Après 15 jours, enlever encore 1 palme sur 2.

Après 15 jours encore, enlever le reste des palmes.

Les palmes enlevées sont évacuées vers la zone de collecte des déchets verts, hors du périmètre de la pépinière. 



\section{Plantation}

La préparation du terrain de la future plantation de palmier est d'une importance capitale. En effet, la durée de vie économique d'une palmeraie est de 20 à 30 ans. Il s'agit, même pour 5 hectares, d'un investissement important.

Auparavant, il est bon de visiter la future parcelle avec un conseiller agricole afin d'éviter les mauvaises surprises. Lidéal reste de développer cette activité dans le cadre d'un plan de développement concerté soit au niveau d'une coopérative soit à un niveau plus général. Les nécessaires études de faisabilité et les études d'impact peuvent être effectuées à ce niveau.

Une parcelle mal préparée et mal plantée ne pourra jamais avoir les rendements escomptés.

Les éléments proposés concernent la localisation de la parcelle, la préparation du terrain, la délimitation de la plantation, le piquetage des lignes de base, des lignes de plantation et de l'emplacement des palmiers, le dégagement des lignes de plantation et enfin la mise en place des plants.

Chaque fois que nécessaire, deux cas seront étudiés : une plantation en extension sur forêt et une replantation derrière palmeraie. 


\section{Fiche 17. Localisation de la parcelle}

Cette fiche concerne le cas d'une création de parcelle en extension sur forêt dégradée ou sur vieille jachère.

La parcelle doit être située à moins de $250 \mathrm{~m}$ d'une piste carrossable en toute période de l'année ou d'une voie bitumée.

Le terrain doit être plat ou faiblement pentu (moins de $5 \%$ ).

Il ne doit pas y avoir de latérite ou de graviers à moins d'1,5 m de profondeur.

Le sol ne doit pas se fendre en saison sèche (cas des sols trop argileux).

Il faut éviter les sols épuisés par plusieurs cycles de cultures vivrières à tubercules ou racines. 


\section{Fiche 18. Préparation du terrain sur forêt dégradée ou vieille jachère}

IV Petit matériel, semences et main-d'œuvre nécessaires

- Machettes, limes, daba, tronçonneuse en location

- 50 à $60 \mathrm{~kg}$ de semences de Pueraria phaseolides.

Tableau 18.1. Main-d'œuvre nécessaire pour 5 ha.

\begin{tabular}{lcc}
\hline Activité & Main-d'œuvre (homme.jour) & Tronçonneuse (heure) \\
\hline Abattage sous bois & 20 & \\
\hline Abattage des arbres & 30 & $100 \mathrm{~h}$ \\
\hline Brûlage abattis & 2 & \\
\hline Débitage des troncs & 70 & $210 \mathrm{~h}$ \\
\hline Rabattage & 30 & \\
\hline Semis Pueraria & 10 & \\
\hline Brûlage final & 3 & \\
\hline
\end{tabular}

\section{Succession des opérations}

\section{Attention}

Il faut commencer les travaux à la fin du mois de septembre de l'année -1 au plus tard pour une plantation en juin de l'année 1.

Abattre le sous-bois à la machette (figure 18.1).

Couper et mettre de côté 800 piquets de 1,5 m de longueur.

Abattre les arbres à la tronçonneuse (figure 18.2).

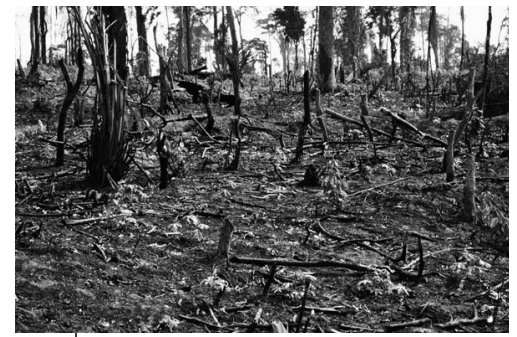

Figure 18.1.

Nettoyage du sous-bois.

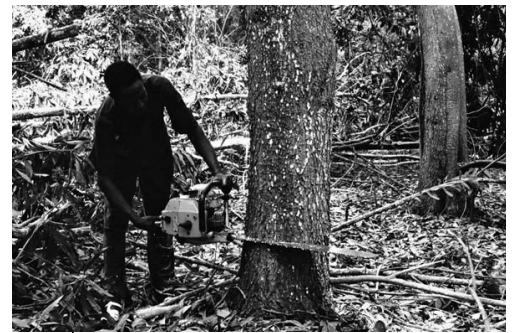

Figure 18.2.

Abattage des arbres à la tronçonneuse. 
Brûler rapidement l'abattis.

Débiter les arbres abattus dont le tronc a un diamètre supérieur à $50 \mathrm{~cm}$. Ce travail doit être achevé au plus tard mi-février de l'année 0 (figure 18.3).

Rabattre complémentairement mi-mars à la machette en prenant bien soin de laisser une bande de sol presque nu de $3 \mathrm{~m}$ de large sur tout le pourtour de la parcelle, qui constituera le pare-feu.

Semis de la plante de couverture, par exemple, Pueraria phaseolides :

- poquets distants de $1 \mathrm{~m}$;

- donner un léger coup de daba sur le sol (cavité de $1 \mathrm{~cm}$ de profondeur) (figure 18.4);

- jeter 10 à 15 graines dans chaque cavité;

- reboucher avec la daba et tasser avec le pied.

Brûler la parcelle fin mars (figure 18.5).

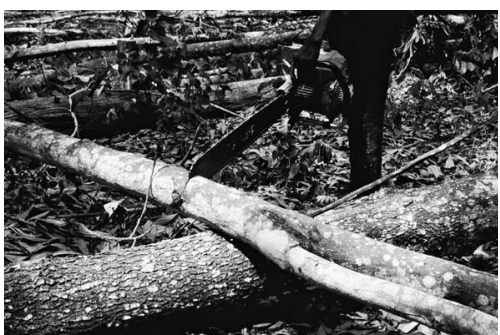

Figure 18.3.

Débitage des troncs

et des grosses branches.

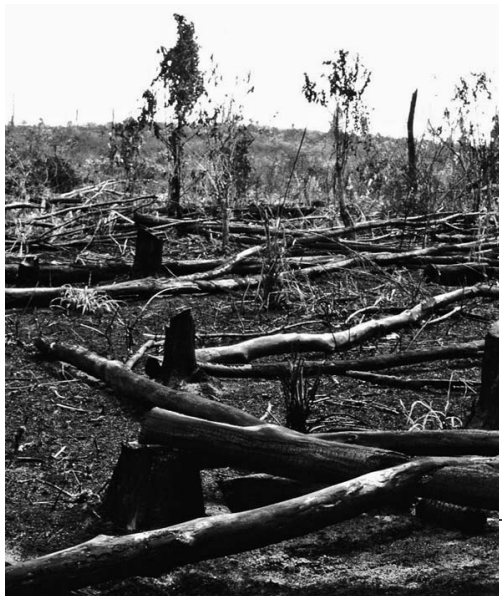

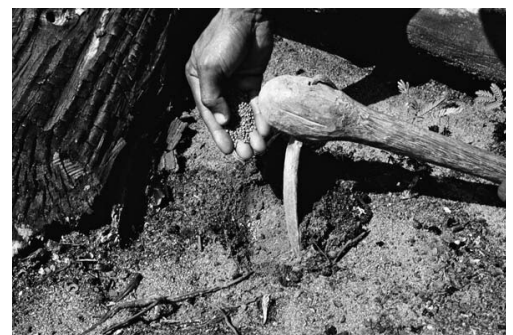

Figure 18.4.

Préparation pour le semis de la plante de couverture.
Figure 18.5.

Brûlage de l'abattis. 


\section{II) Concernant le brûlage}

Bien qu'interdit pour la préparation des plantations commerciales, l'utilisation du feu comme moyen de nettoyer le terrain est admis pour les petites exploitations en raison de son faible coût et de son usage souvent ancestral.

Les opérations de brûlage doivent toujours être conduites sous une forte surveillance afin d'éviter de mettre le feu aux parcelles adjacentes, qu'elles soient plantées ou non.

Les limites du terrain à brûler doivent être débarrassées de tout débris végétal.

Il faut toujours commencer le feu par la bordure sous le vent.

Il faut aussi veiller à ne pas embraser l'ensemble de la parcelle en une seule fois, sinon, le feu deviendrait vite incontrôlable.

Il faut laisser un gardiennage en place tant qu'il y a un risque de propagation des flammes en dehors du périmètre. 


\section{Fiche 19. Préparation du terrain pour une replantation derrière palmeraie}

\section{I| Main-d'œuvre}

- Abattage palmiers : (pour mémoire).

- Rabattage : $30 \mathrm{HJ}$.

- Semis couverture : $10 \mathrm{HJ}$.

- Brûlage final : $3 \mathrm{HJ}$.

\section{Petit matériel et semences nécessaires}

- Machettes, ciseaux, limes, daba

- 50 à $60 \mathrm{~kg}$ de Pueraria phaseolides.

\section{ID Détail des travaux à réaliser}

Débuter les travaux fin juillet de l'année $\mathbf{- 1}$ au plus tard pour une replantation en juin de l'année $\mathbf{1}$.

Abattre les palmiers en sectionnant les racines avec un ciseau de récolte, une pelle tranchante ou une hache (figure 19.1).

Faire tomber les palmiers autant que possible vers l'andain.

Couper toutes les feuilles et les ranger sur les stipes (figure 19.2).

\section{Attention}

Le plus souvent, ces opérations sont réalisées par le propriétaire ou un contractuel pour s'achever par l'extraction du vin de palme (figure 19.3). Ces opérations doivent impérativement être achevées trois mois avant la plantation.

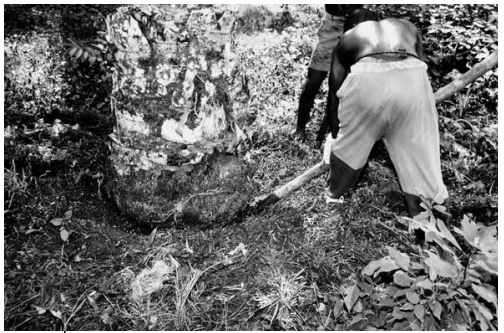

Figure 19.1.

Abattage des vieux palmiers.

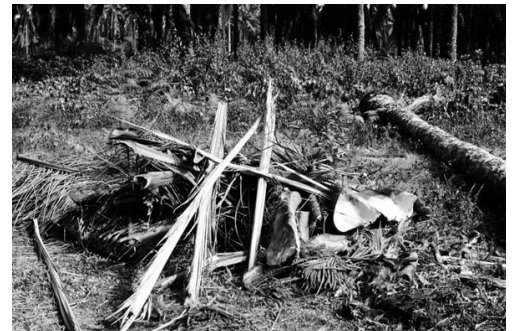

Figure 19.2.

Rangement des feuilles sur les stipes. 
Rabattre à la machette en prenant bien soin de laisser une bande de sol presque nu de $3 \mathrm{~m}$ de large sur tout le pourtour de la parcelle (futur pare-feu).

Semis de la plante de couverture (Pueraria phaseolides)

- semis en poquets distants de $1 \mathrm{~m}$;

- donner un léger coup de daba sur le sol (cavité de $1 \mathrm{~cm}$ de profondeur);

- jeter 10 à 15 graines dans la cavité (figure 19.4);

- reboucher avec la daba et tasser avec le pied.

Brûlage final de la parcelle fin mars.

\section{Attention}

L'extraction du vin de palme est très souvent l'occasion de nombreux départs de feu qui ne sont pas faciles à maîtriser en raison du degré important de salissure des parcelles à ce moment-là (voir Fiche 18 : «Concernant le brûlage» page 65).

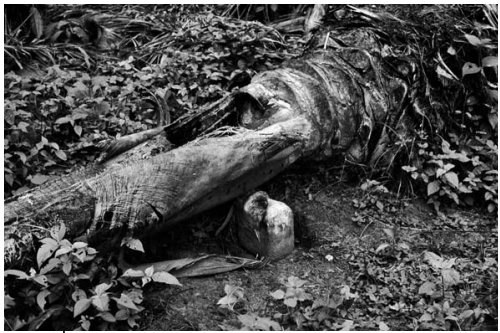

Figure 19.3.

Extraction du vin de palme : le liquide coule goutte à goutte, il faut toute une nuit pour recueillir 1 à 2 litres de vin de palme.

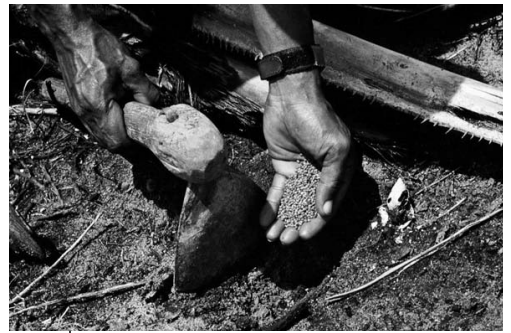

Figure 19.4.

Semis de la plante de couverture. 


\section{Fiche 20. Fabrication et utilisation du gabarit d'angle droit}

ID Main-d'œuvre

- 2 personnes.

|D Petit matériel

- Grosse ficelle : environ $13 \mathrm{~m}$

- 3 piquets

- 1 double mètre.

\section{ID Fabrication du gabarit}

Prendre une grosse ficelle d'au moins 12,5 m (figure 20.1A).

Couper 3 petits piquets : diamètre 1 à $1,5 \mathrm{~cm}$, longueur $30 \mathrm{~cm}$.

Attacher solidement une extrémité de la ficelle à un des piquets. Il devient le piquet 1 .

Enfoncer le piquet dans le sol.

Enfoncer le second piquet à $3 \mathrm{~m}$ exactement du premier.

Attacher solidement la ficelle bien tendue à ce second piquet.

Enfoncer le troisième piquet à exactement $5 \mathrm{~m}$ du piquet 2 .

Attacher solidement la ficelle bien tendue au troisième piquet.

Tendre la ficelle et faire un nœud à exactement $4 \mathrm{~m}$ du piquet 3 .

Déplanter les piquets sans détacher la ficelle.

Le gabarit achevé mesure $12 \mathrm{~m}$ de long et présente une extrémité munie d'un piquet et l'autre d'un nœud.

\section{ID Utilisation du gabarit pour un angle droit}

Il est nécessaire d'avoir un assistant.

Planter le piquet 1 bien verticalement dans le sol (figure 20.1B).

Planter le piquet 2 bien verticalement dans le sol, ficelle bien tendue.

Tenir le nœud du bout de la ficelle contre le piquet 1 .

Demander à l'assistant de tendre sans brutalité la ficelle en se déplaçant et en tenant le piquet 3 bien vertical. 
Lorsque les deux brins sont tendus, planter le piquet 3.

Les lignes passant par les piquets 1 et 2 , d'une part, et les piquets 1 et 3 , d'autre part, sont perpendiculaires.

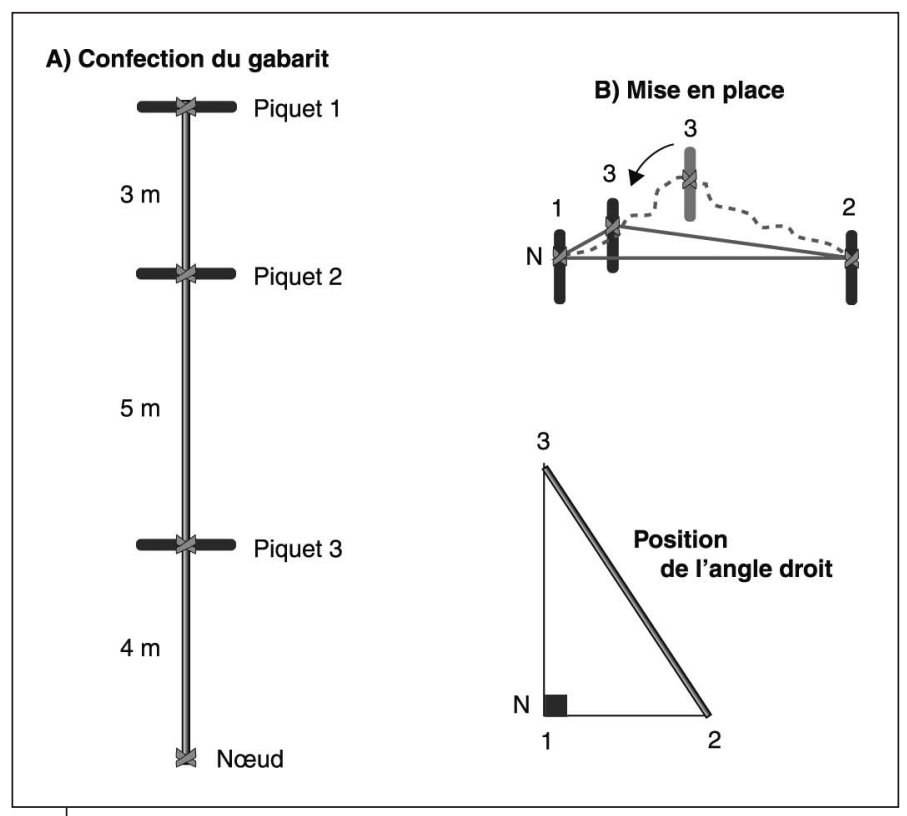

Figure 20.1.

Confection et utilisation du gabarit d'angle droit. 


\section{Fiche 21. Délimitation de la parcelle et ligne de base dans le cas d'une extension sur forêt}

\section{Main-d'œuvre}

- $15 \mathrm{HJ}$.

- Minimum 3 personnes : il faut 5 jours à 3 personnes pour accomplir ce travail.

\section{ID Petit matériel}

- Machette

- Bambous minces en quantité suffisante

- Bandes de tissu clair de 2 x $50 \mathrm{~cm}$

- Une grosse ficelle de 23,4 $\mathrm{m}$ avec un nœud tous les 7,8 m

- Un double mètre.

\section{ID Succession des travaux à réaliser}

Couper des bambous minces de $3 \mathrm{~m}$ de longueur.

Planter 1 bambou à chaque coin du polygone formé par le terrain préparé. On matérialise ainsi la lisière de la parcelle.

Planter d'autres bambous à une distance de 4,5 $\mathrm{m}$ des bambous précédents vers le centre de la parcelle.

$\mathrm{Au}$ sommet de ces bambous, fixer un morceau de tissu clair. On matérialise ainsi la bordure de la parcelle (figure 21.1).

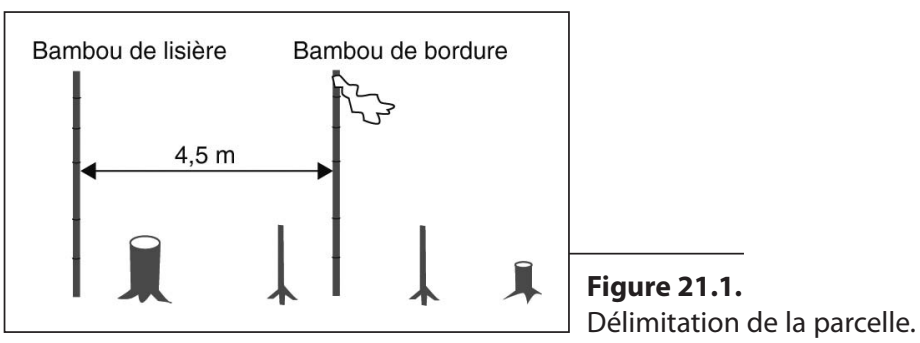

Il ne faudra pas planter de palmiers dans la zone comprise entre la bordure et la lisière.

Récupérer les 800 jalons (piquet de bois ou de bambou de 1,5 m de long qui servira à indiquer l'emplacement des lignes et des palmiers) préparés précédemment. 
Chercher et matérialiser la plus longue diagonale du polygone (figure 21.2). Ce sera la ligne de base.

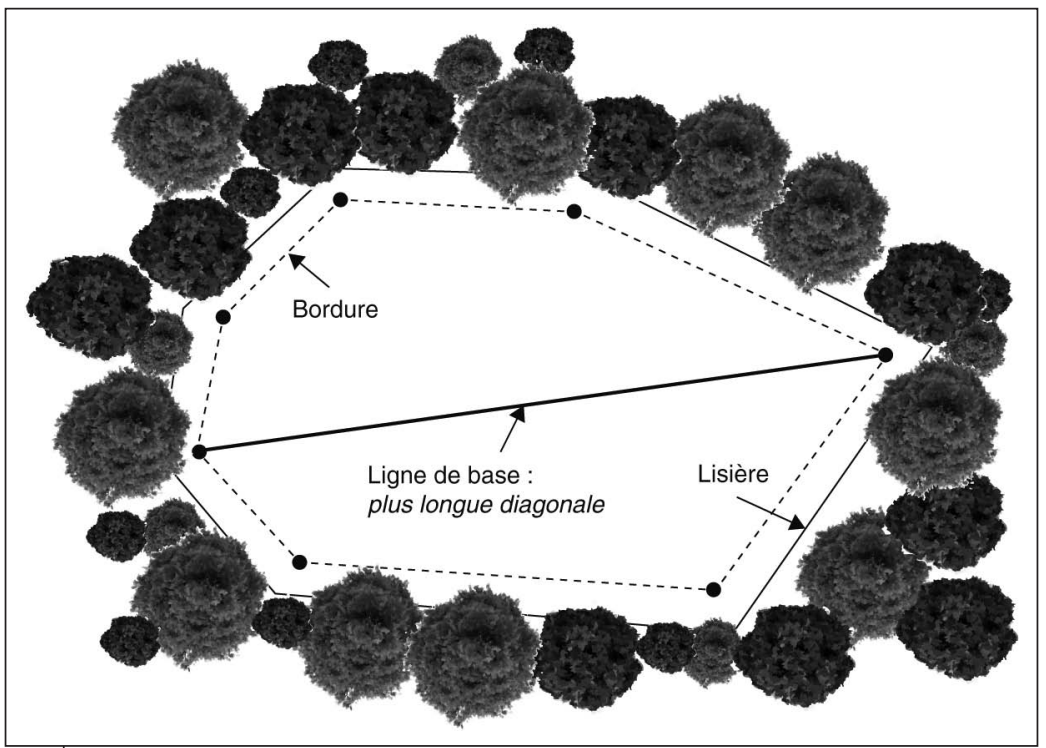

Figure 21.2.

Recherche de la plus longue diagonale.

Choisir une de ses extrémités.

Remplacer le bambou de bordure par un jalon.

Le long de la ligne de base, planter un jalon tous les 7,8 $\mathrm{m}$ en vérifiant son alignement avec le bambou marqué placé à l'autre extrémité de la ligne de base (figure 21.3).

\section{Attention}

Toutes les opérations de piquetage se font à 3 personnes. La première personne fait la visée, les deux autres transportent et positionnent les jalons. 


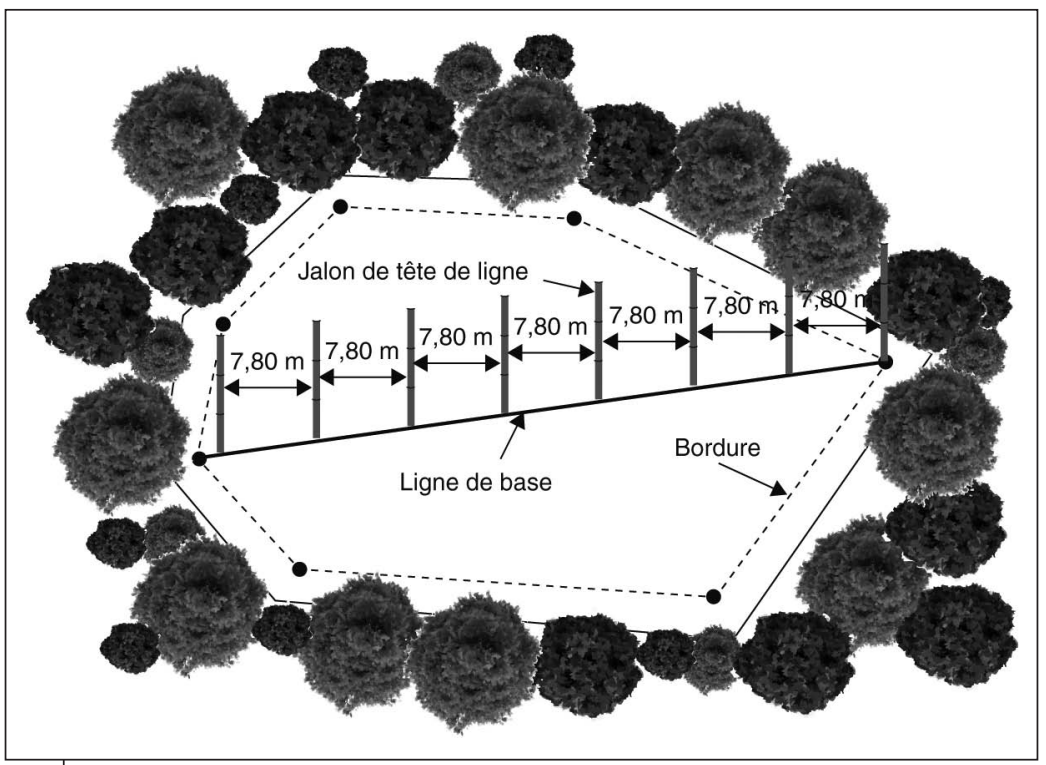

Figure 21.3.

Piquetage de la ligne de base. 


\section{Fiche 22. Piquetage des lignes de plantation dans le cas d'une extension sur forêt}

Il faut 3 personnes pour ce travail.

Se munir du gabarit d'angle droit.

Planter le piquet 1 à la place du premier jalon de la ligne de base (figure 22.1).

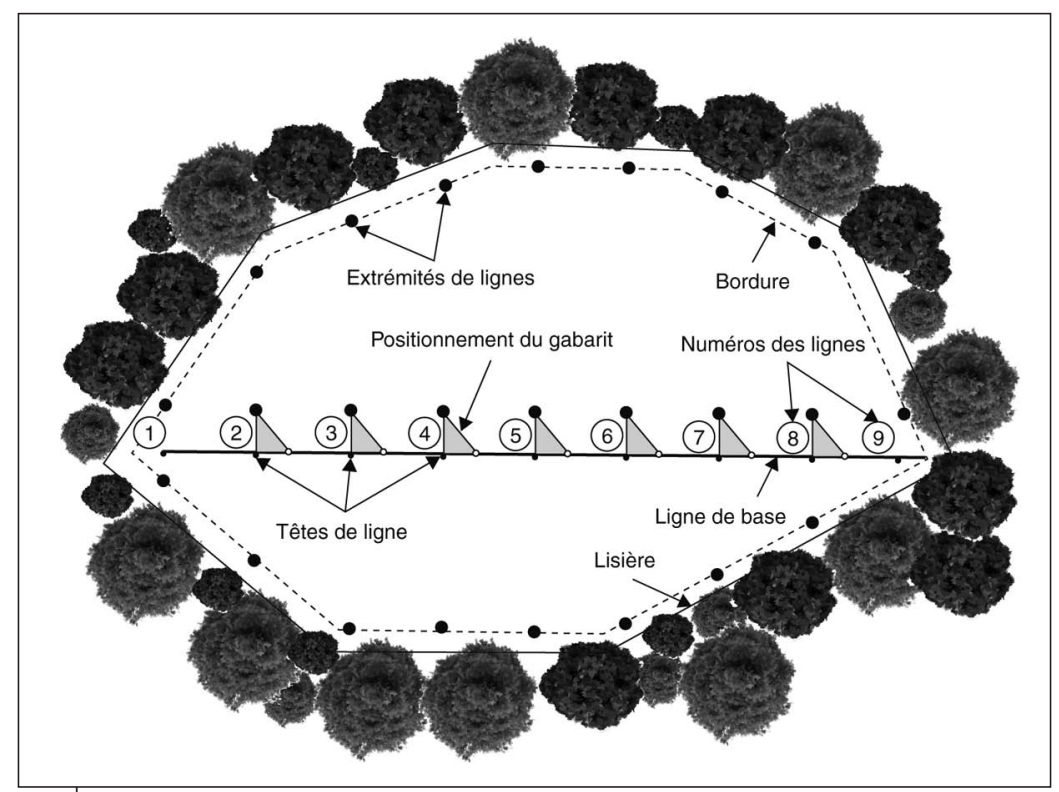

Figure 22.1.

Piquetage des têtes et des extrémités de lignes.

Ensuite : planter le piquet 2 sur la ligne de base, ficelle bien tendue. Suivre le mode d'utilisation du gabarit pour planter le piquet 3 (voir Fiche 20).

Remplacer le piquet 3 par un jalon.

Remettre le jalon de ligne de base à la place du piquet 1 .

La première ligne de plantation est définie par l'alignement du premier jalon de la ligne de base et du jalon remplaçant le piquet 3 .

Recommencer avec le second jalon de la ligne de base pour définir la seconde ligne de plantation. 
Ainsi de suite jusqu'au dernier jalon de la ligne de base.

En s'alignant sur les deux jalons posés pour la dernière ligne de plantation, poser un jalon à l'intersection des lignes imaginaires entre les bambous de bordure et la ligne de plantation d'un côté puis de l'autre.

Recommencer la même opération pour l'avant-dernière ligne de plantation.

Ainsi de suite jusqu'à la dernière ligne de plantation. 


\section{Fiche 23. Piquetage des palmiers dans le cas d'une extension sur forêt}

\section{||D Main-d'œuvre}

- $40 \mathrm{HJ}$, y compris le piquetage des lignes de plantation

- Minimum 3 personnes.

\section{ID Petit matériel}

- Grosse ficelle

- Un double mètre.

\section{Détail des travaux à réaliser}

Il faut trois personnes pour cette opération.

Se munir d'une grosse ficelle d'environ $25 \mathrm{~m}$ de long.

Faire un nœud à une extrémité.

Faire un second nœud à 4,5 $\mathrm{m}$ du premier.

Faire un troisième nœud à $9 \mathrm{~m}$ du second.

Faire un quatrième nœud à $9 \mathrm{~m}$ du troisième.

Pour le piquetage des emplacements des palmiers de la ligne 1 : se mettre derrière les jalons de la ligne 1 (figure 23.1).

Demander aux assistants de tendre la ficelle dans l'axe de la ligne, le long des jalons en positionnant le second nœud au pied du premier jalon.

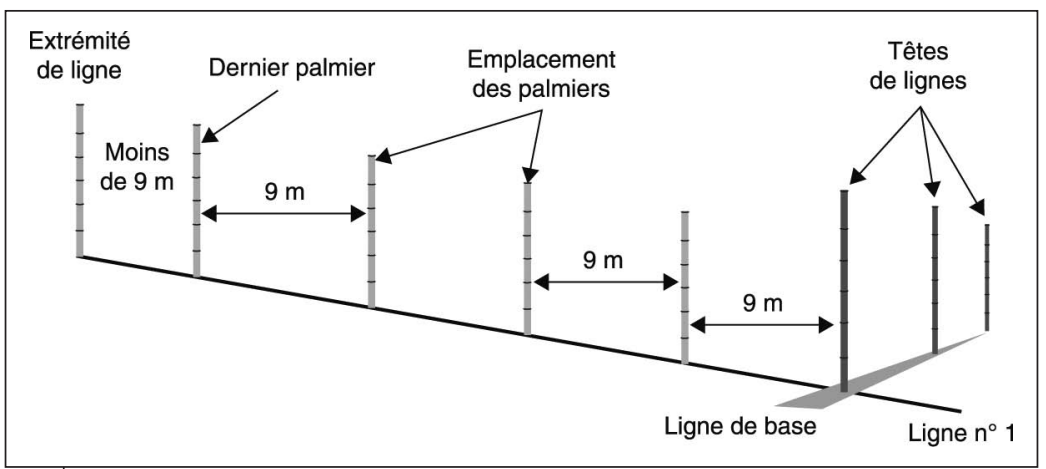

Figure 23.1.

Piquetage de la première ligne de palmiers. 
Positionner un jalon tous les $9 \mathrm{~m}$ en s'aidant de la ficelle.

Indiquer par gestes aux assistants la position exacte des emplacements des palmiers en restant exactement dans l'axe de la ligne défini par les 2 premiers jalons.

Faire de même vers l'autre extrémité de la ligne.

Enlever le jalon situé à $4 \mathrm{~m}$ de la ligne de base.

Pour le piquetage des emplacements des palmiers de la ligne 2 : se mettre derrière les jalons de la ligne 2 (figure 23.2).

Demander aux assistants de tendre la ficelle dans l'axe de la ligne, le long des jalons en positionnant le premier nœud au pied du premier jalon.

Positionner un jalon à $4,5 \mathrm{~m}$ puis tous les $9 \mathrm{~m}$ en s'aidant de la ficelle (à partir du second nœud).

Indiquer par gestes aux assistants la position exacte des emplacements des palmiers en restant exactement dans l'axe de la ligne défini par les 2 premiers jalons.

Faire de même vers l'autre extrémité de la ligne.

Enlever le jalon de la ligne de base et le jalon situé à $4 \mathrm{~m}$ de la ligne de base.

Lignes 3, 5, 7 etc. (lignes impaires) : recommencer comme pour la ligne 1.

Lignes 4, 6, 8 etc. (lignes paires) : recommencer comme pour la ligne 2.

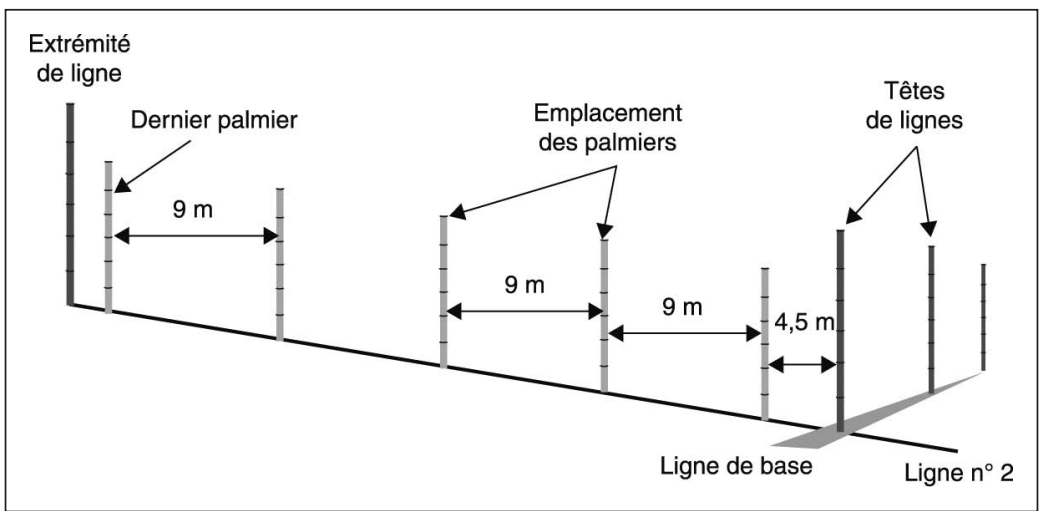

Figure 23.2.

Piquetage de la seconde ligne de palmiers. 


\section{Fiche 24. Piquetage des lignes et emplacement des arbres pour une replantation derrière palmeraie}

\section{Main-d'œuvre}

- $40 \mathrm{HJ}$

- Minimum 3 personnes.

\section{II) Petit matériel}

- Une corde mince de $18 \mathrm{~m}$ avec un nœud tous les $9 \mathrm{~m}$

- Un double mètre.

\section{II) Succession des opérations}

Repérer avec des bambous, l'emplacement des anciennes lignes de plantation.

Se placer sur la première ligne de plantation (figures 24.1 et 24.2). Positionner un jalon sur le centre de la souche du premier et du second palmier de la ligne.

Positionner un jalon exactement au milieu entre ces deux jalons.

Enlever les jalons posés sur les souches.

Recommencer sur la ligne suivante jusqu'à la dernière ligne.

Se placer à l'autre extrémité de la dernière ligne de plantation.

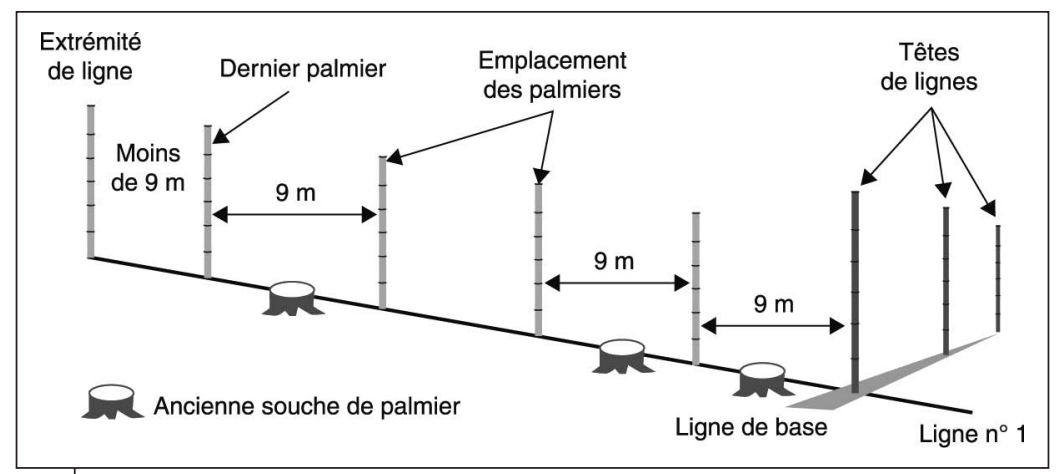

Figure 24.1.

Piquetage de la première ligne de palmiers. 
Positionner un jalon au milieu de la souche de l'avant-dernier et du dernier palmier de la ligne.

Positionner un jalon exactement au milieu entre ces deux souches.

Enlever les jalons posés sur les souches.

Recommencer sur la ligne suivante jusqu'à la première ligne.

Se placer sur la première ligne de plantation.

Positionner un jalon tous les $9 \mathrm{~m}$ sur la ligne en s'aidant de la corde marquée.

Vérifier l'alignement en s'aidant des jalons de tête et de fin de ligne.

Sur les lignes, mettre un palmier sortant quand le palmier de première génération était rentrant et réciproquement.

Recommencer sur la ligne suivante jusqu'à la dernière ligne.

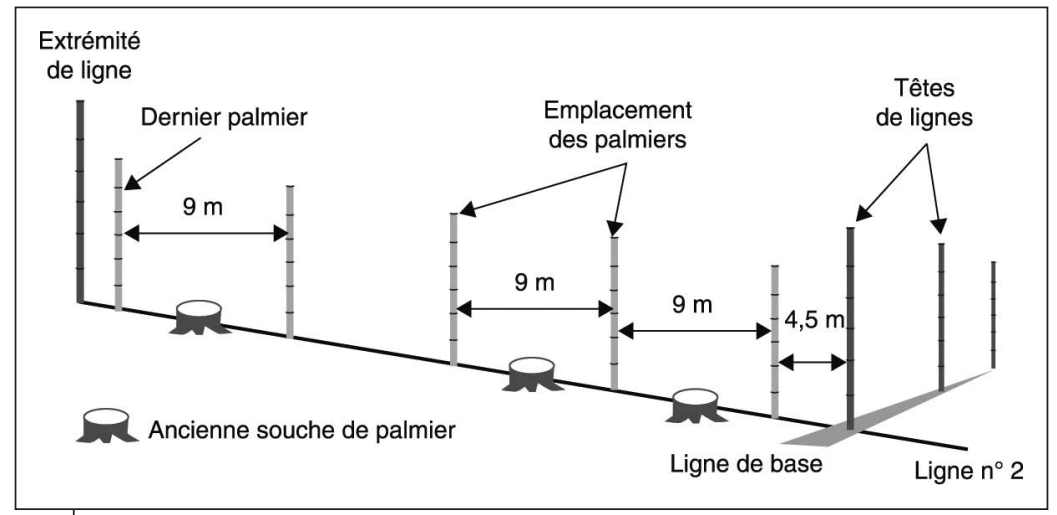

Figure 24.2.

Piquetage de la seconde ligne de palmiers. 


\section{Fiche 25. Dégagement des lignes de palmiers}

\section{II) Main-d'œuvre et petit matériel}

- Maximum $40 \mathrm{HJ}$

- Location de tronçonneuse : 200 heures.

\section{II) Extension sur forêt}

Dégager à la machette et/ou à la tronçonneuse un sentier de $1 \mathrm{~m}$ de large au milieu d'un interligne sur deux.

Dégager à la machette et/ou à la tronçonneuse un rond de $1,5 \mathrm{~m}$ de rayon autour de l'emplacement du futur palmier.

Débarder tous les bois gênants sur les côtés des ronds et des sentiers. Préparer, si nécessaire une petite plateforme (terrasse manuelle) (figure 25.1).

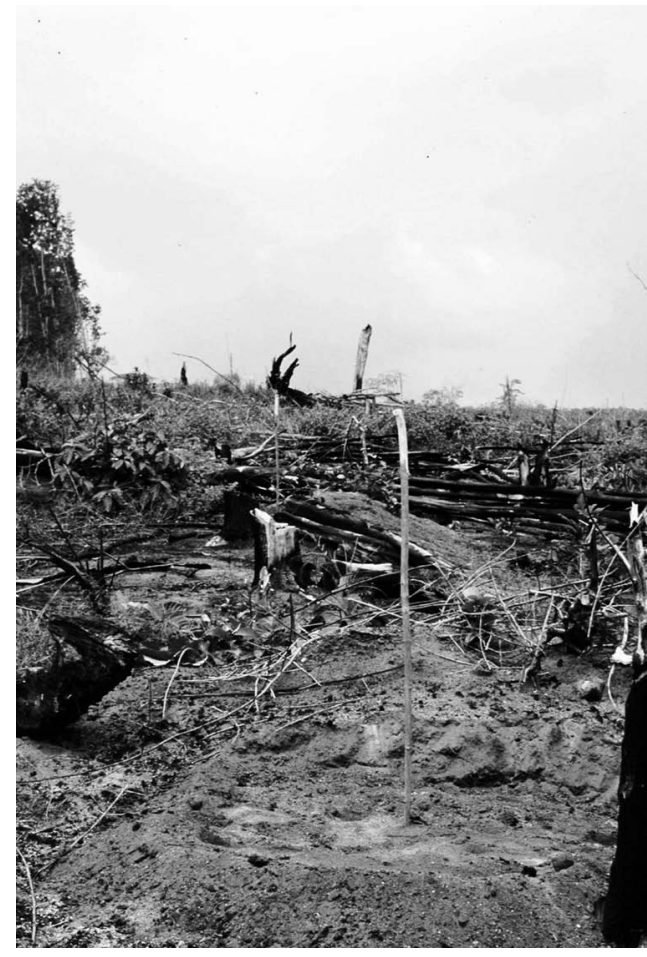

Figure 25.1.

Emplacement de palmier préparé dans le cas d'une extension. 


\section{II) Replantation}

Couper à la tronçonneuse les stipes de palmiers gênant le passage sur la ligne de plantation.

Débarder les portions de stipes gênantes sur les côtés des ronds et des sentiers.

Dégager à la machette et/ou à la tronçonneuse un rond de $1,5 \mathrm{~m}$ de rayon autour de l'emplacement du futur palmier (figure 25.2).

Préparer une petite plateforme (terrasse manuelle).

\section{Attention}

Ces opérations ne sont à réaliser que si elles sont nécessaires.

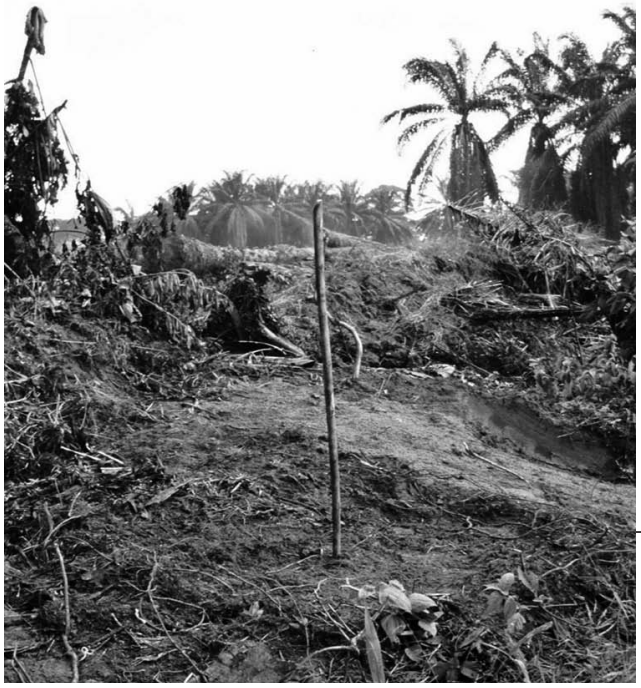

Figure 25.2.

Emplacement de palmier préparé dans le cas d'une replantation. 


\section{Fiche 26. Mise en place des plants}

Si les plants ne proviennent pas de la pépinière conduite par le planteur lui-même, il faut veiller à s'approvisionner auprès d'un fournisseur de matériel végétal agréé qui offre toutes les garanties de légitimité et de potentiel de production. Le matériel tout-venant est impropre à la réalisation d'une plantation de qualité en raison de la présence d'anormaux, de palmiers illégitimes et de palmiers consanguins.

\section{Main-d'œuvre}

- Transport des plants : $15 \mathrm{HJ}$

- Trouaison et mise en terre : 20 à $50 \mathrm{HJ}$

- Grillage spiral : $15 \mathrm{HJ}$.

\section{ID Petit matériel}

- Brouette, charrette à bras, tracteur agricole selon les besoins

- Ficelle

- Machette, daba, sécateur

- $72 \mathrm{~m}$ de grillage.

- Une paire de tenailles pour couper le grillage

- Une ou plusieurs paires de gants pour le portage des plants.

\section{ID Transport des plants}

Préparer les plants en liant les feuilles entre elles avec un bout de ficelle ou de liane fine vers le milieu de leur longueur (figure 26.1).

Si la hauteur des plants dépasse 1,75 m (hauteur d'homme), raccourcir les feuilles en sectionnant leur extrémité à cette hauteur avec un sécateur ou une machette bien aiguisée.

Effectuer le transport :

- si la pépinière est très éloignée du lieu de plantation (plus de $5 \mathrm{~km}$ ), faire le transport par camion, camionnette ou tracteur agricole;

- si la pépinière est peu éloignée du lieu de plantation (moins de $5 \mathrm{~km}$ ), faire le transport avec une charrette attelée ou à bras (figure 26.2).

Déposer les plants en lisière de la plantation.

Transporter les plants au pied de chaque jalon.

La meilleure méthode est de transporter les plants en brouette. Il est ainsi possible de charger de 4 à 5 plants par voyage. La manutention 
des plants se fait en agrippant ceux-ci par le collet d'une main, l'autre retenant le sac par le côté ou par en dessous.

$\mathrm{Ne}$ jamais transporter le plant en l'agrippant par les feuilles ou en traînant le sac par terre.

Ne jamais mettre les plants en position couchée pour ce transport.

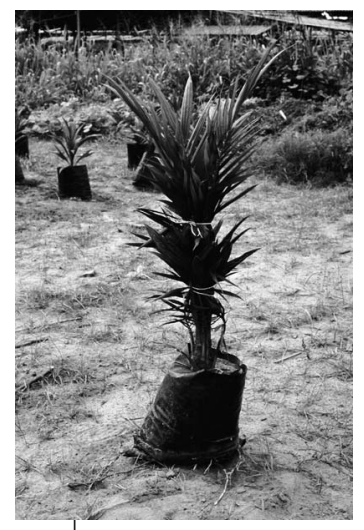

Figure 26.1.

Préparation du plant au transport.

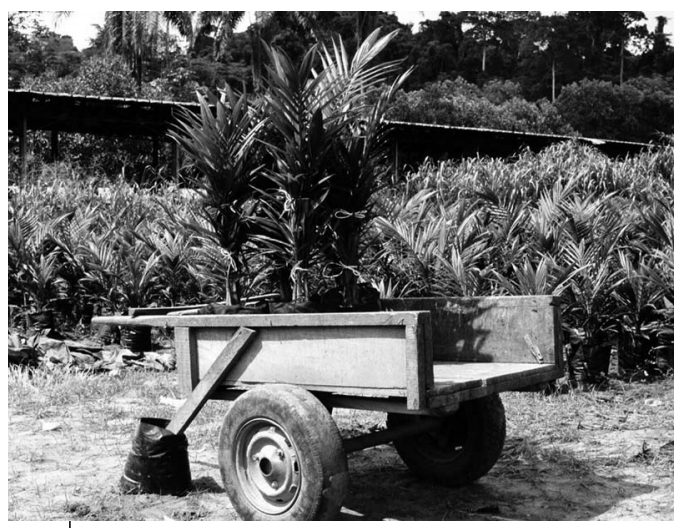

Figure 26.2.

Chargement d'une charrette.

\section{II) Trouaison}

Enlever le jalon.

Faire un trou de $40 \mathrm{~cm}$ de diamètre et $40 \mathrm{~cm}$ de profondeur à la daba. Mettre la terre de surface d'un côté (à gauche sur la photo) et la terre du fond de l'autre côté (figure 26.3).

Vérifier les dimensions du trou avec un gabarit (baguette de bois ou de bambou de $40 \mathrm{~cm}$ de long).

\section{ID Mise en terre}

Incliner le sac contenant le palmier sur le côté.

Découper le fond du sac avec une machette bien aiguisée (figure 26.4).

Détacher et jeter le fond.

Prendre le palmier par en dessous.

Déposer le plant délicatement au fond du trou.

Vérifier que le collet du plant se trouve bien au niveau du sol (figure 26.5).

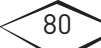


Faire glisser le sac vers le haut par dessus les feuilles (figure 26.6).

Combler l'espace entre la motte et la paroi du trou avec la terre de surface.

Tasser soigneusement, mais modérément (figure 26.7).

Récupérer tous les sacs et débris en plastique pour les brûler.

\section{ID Protection contre les rongeurs}

Si le précédent cultural était constitué de cultures vivrières ou si des cultures vivrières intercalaires sont ou ont été installées, il y a un grand risque d'attaques de rongeurs. La lutte préventive utilise des grillages spirales.

Pour chaque palmier il faut prévoir une bande de $1 \mathrm{~m}$ de long et de $10 \mathrm{~cm}$ de large de grillage à poule ou à maille carrée de $1 \mathrm{~cm}$.

Enrouler la bande autour du collet en passant en avant et en arrière des bases des feuilles (figure 26.8). Dégager une zone propre de $3 \mathrm{~m}$ de diamètre autour du jeune plant.

Si le sol est suffisamment argileux, il est possible de protéger le plant en faisant un emplâtre d'argile tout autour du collet.

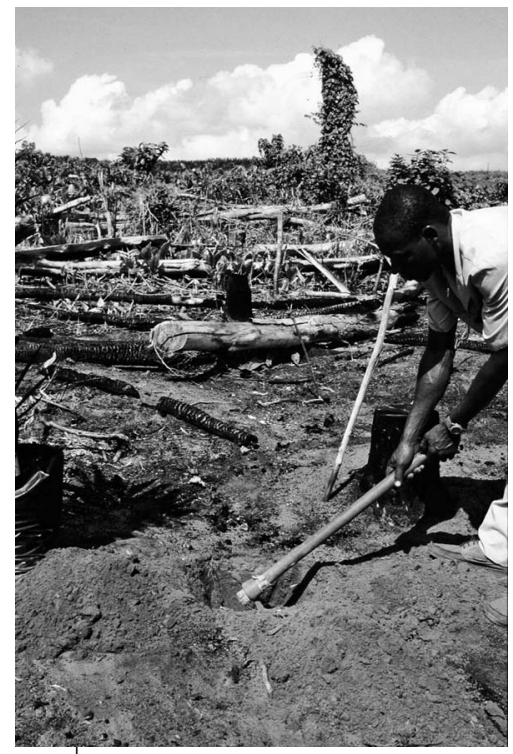

Figure 26.3.

Trouaison.

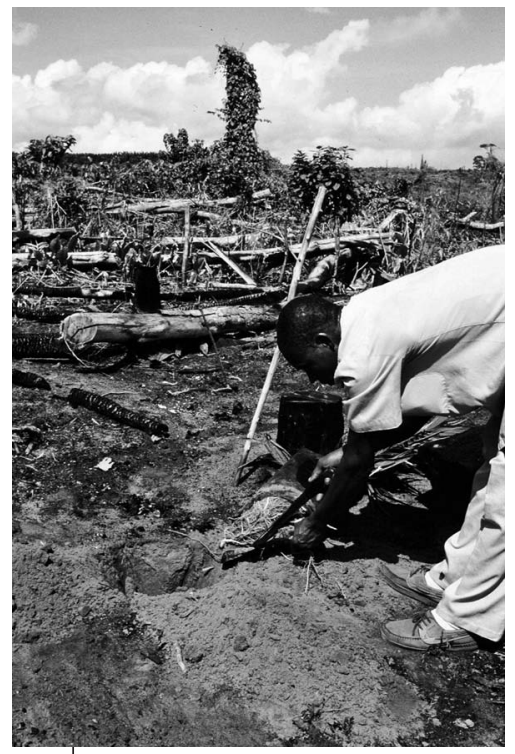

Figure 26.4.

Découpe du fond du sac. 


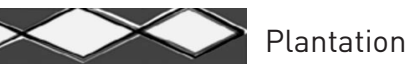

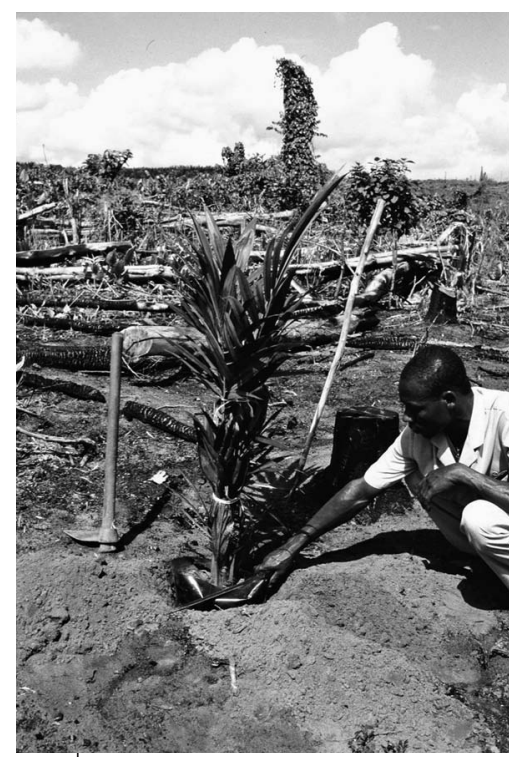

Figure 26.5.

Vérification du niveau du collet.

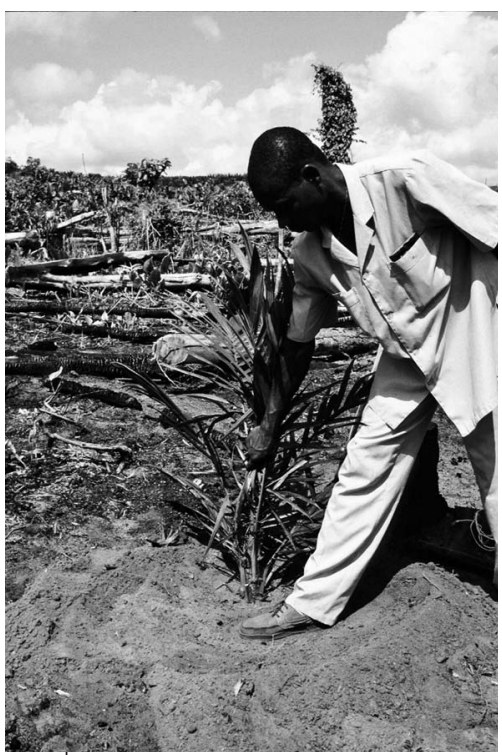

Figure 26.7.

Tassement du sol autour

de la motte de pépinière.

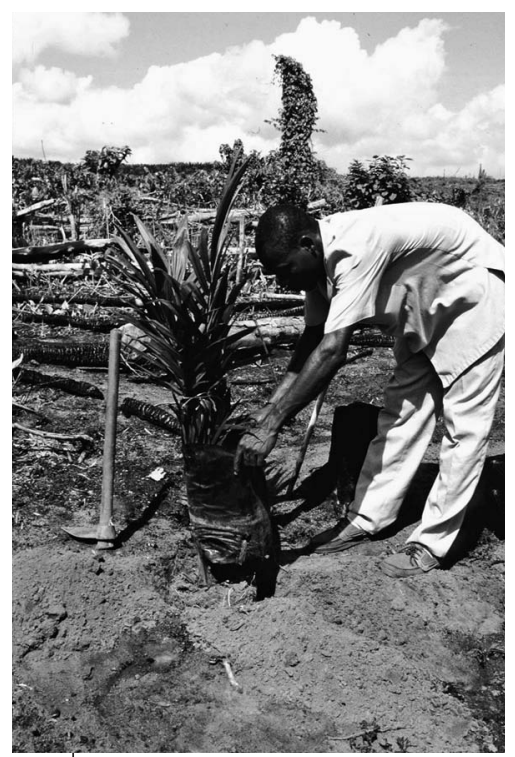

Figure 26.6.

Enlèvement du sac de pépinière.

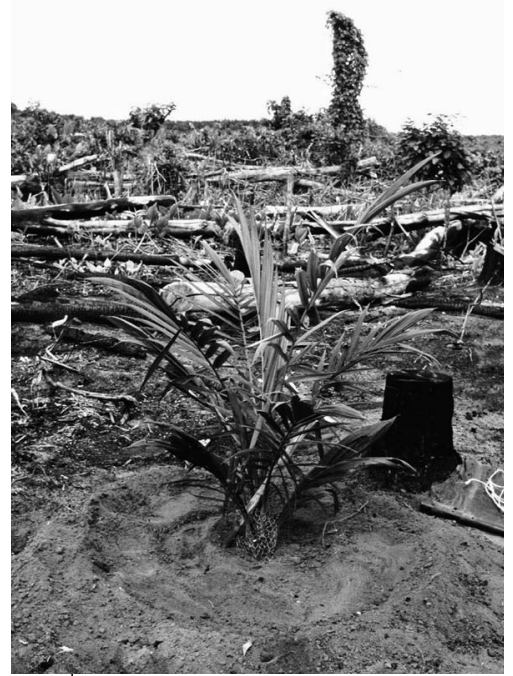

Figure 26.8.

Jeune palmier bien protégé. 


\section{Jeunes cultures}

La phase immature des palmiers dure de 2 à 4 années selon les conditions pédoclimatiques. Les tout-premiers régimes ne sont pas économiquement intéressants.

Dans le schéma traditionnel d'exploitation en Afrique de l'Ouest, la mise en récolte est prévue à 3 ans révolus.

Calendrier prévisionnel :

- plantation au champ, juin 2014;

- stade jeune culture, de juin 2014 à mai 2017;

- mise en récolte, juin 2017.

Ce stade de la culture mérite tous les soins du planteur car une bonne conduite de la plantation pendant cette période sera la garantie d'une bonne productivité dans le futur.

Lattention du planteur est donc attirée sur l'entretien de sa parcelle et de ses palmiers, les précautions à prendre s'il conduit des cultures intercalaires, la surveillance phytosanitaire et la fumure.

\section{Attention}

Par convention, l'année 1 commence à la date de plantation et s'achève l'année suivante à la date anniversaire de cette plantation. L'année 2 commence à la date du premier anniversaire de la plantation pour s'achever à la date anniversaire de l'année suivante. Il en est de même pour les années suivantes. 


\section{Fiche 27. Entretien de la parcelle: ronds et couverture}

\section{Entretien des ronds}

\section{Besoins en matériel}

- 1 machette légère

- 1 daba

- 1 gabarit pour l'année 1 .

Temps de travaux

Année 1

- 1 Homme-Jour (HJ) / ha x 5 ha $=5 \mathrm{HJ} \times 6$ passages $/$ an $=30 \mathrm{HJ}$.

\section{Années 2 et 3}

- $1 \mathrm{HJ} /$ ha $\mathrm{x} 5 \mathrm{ha}=5 \mathrm{HJ} \times 4$ passages $/$ an $=20 \mathrm{HJ}$.

Détail des travaux à réaliser

\section{Pendant l'année 1}

Utiliser une machette légère et/ou une daba.

Se protéger les jambes en utilisant un bâton tenu en main de façon à couper la trajectoire de la machette.

Fabriquer un gabarit avec un bois ou un bambou fin long de 1,5 m.

Nettoyer jusqu'à sol nu, une surface de rayon égal au gabarit tout autour du jeune palmier.

Enlever les tiges des plantes grimpantes ou de Pueraria qui auraient pu envahir le palmier.

Vérifier le positionnement du grillage spirale si cette protection contre les rongeurs a été installée et le remettre en place si nécessaire.

Vérifier la dimension du rond avec le gabarit.

Procéder palmier après palmier, puis ligne après ligne.

Faire le premier entretien le lendemain de la plantation.

Faire les autres passages d'entretien des ronds tous les deux mois (figure 27.1).

\section{Pendant l'année 2 et l'année 3}

Utiliser une machette légère et/ou une daba.

Se protéger les jambes en utilisant un bâton tenu en main. 
Nettoyer, jusqu'à obtenir sol nu, toute la surface jusqu'à l'aplomb de l'extrémité des feuilles tout autour du jeune palmier.

Enlever les tiges des plantes grimpantes qui auraient pu envahir le jeune palmier.

Procéder palmier par palmier, puis ligne après ligne.

Faire un passage d'entretien des ronds tous les 3 mois (figures 27.2 et 27.3).

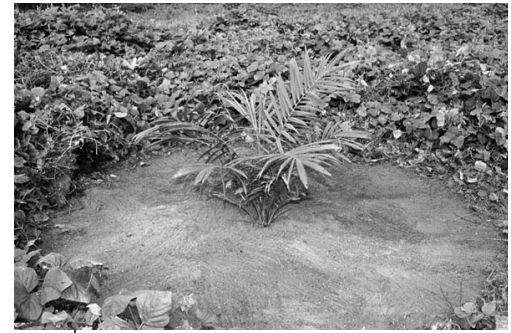

Figure 27.1.

Jeune palmier d'année 1.

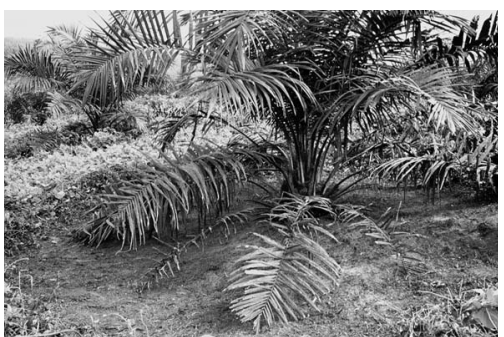

Figure 27.3.

Jeune palmier d'année 3 .

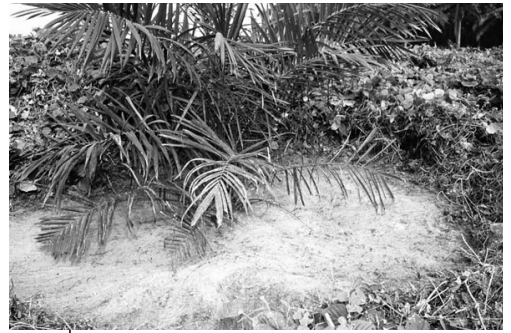

Figure 27.2.

Jeune palmier d'année 2 .

\section{II) Entretien de la couverture}

Besoins en matériel

- 1 machette lourde.

Temps de travaux (minimum)

- $1 \mathrm{HJ} /$ ha $\times 5 \mathrm{ha}=5 \mathrm{HJ} \times 2$ passages $/$ an $=10 \mathrm{HJ}$.

Détail des travaux à réaliser

Utiliser une machette lourde. 
Se protéger les jambes avec un bâton tenu en main.

Couper les repousses d'arbres, d'arbustes et de végétaux buissonnants au ras du sol.

Rabattre légèrement le Pueraria phaseolides près des ronds des palmiers (figure 27.4).

Faucher les graminées et les mauvaises herbes à $20 \mathrm{~cm}$ de hauteur minimum.

Procéder interligne après interligne.

Faire un passage tous les 6 mois.

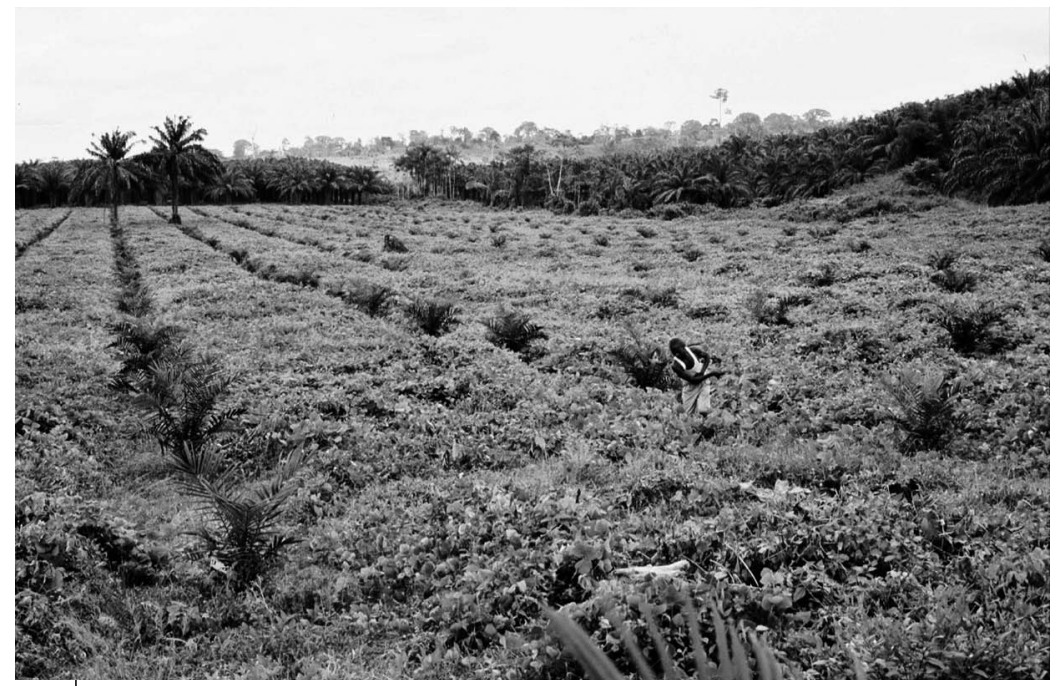

Figure 27.4.

Jeune palmeraie d'année 1 bien entretenue. 


\section{Fiche 28. Extirpation de Chromolaena odorata}

Si la couverture de Pueraria est mal installée, ou après un cycle de cultures vivrières, il est très fréquent que la jeune plantation soit envahie par Chromolaena odorata, souvent appelée «Indépendance» par les agriculteurs (figure 28.1). La croissance de cette mauvaise herbe est extrêmement rapide. La plante peut ainsi former des fourrés impénétrables en quelques mois. Cela favorise le développement des ravageurs des jeunes palmiers comme les rongeurs et les criquets puants. La lutte contre cette plante se fait par voie chimique avant la replantation sur les plantations industrielles. Nous présenterons ici une technique de lutte manuelle.

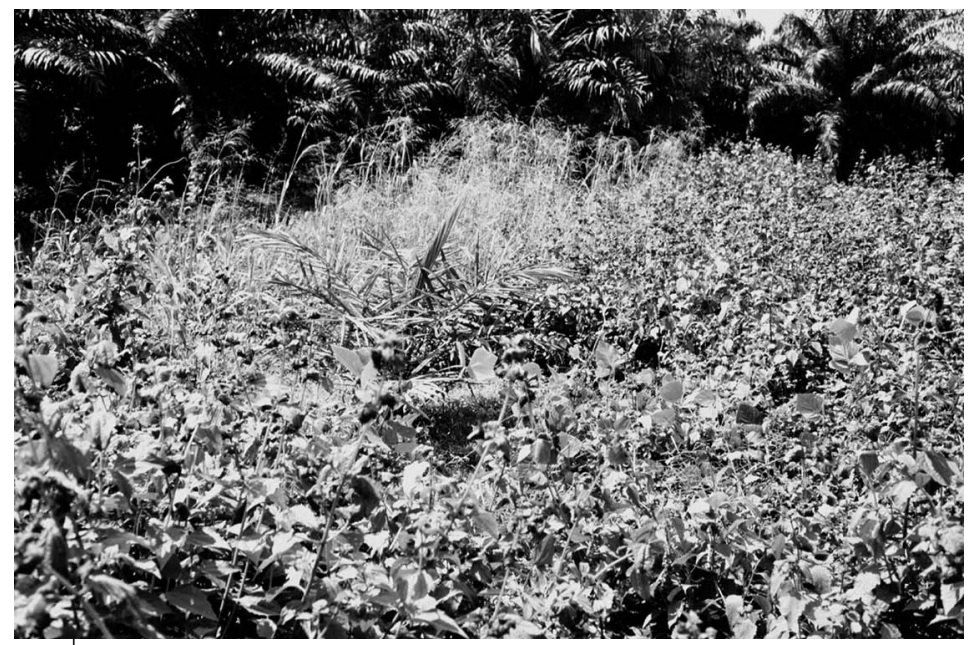

Figure 28.1.

Parcelle de jeunes palmiers avant rabattage du Chromolaena odorata.

II) Besoins en matériel

- 1 machette

- 1 daba

- 1 panier.

Temps de travaux pour une parcelle totalement envahie Rabattage

$1,5 \mathrm{HJ} /$ ha $\mathrm{x} 5 \mathrm{ha}=7,5 \mathrm{HJ}$ 


\section{Premier tour d'extirpation}

$14 \mathrm{HJ}$ / ha x 5 ha $=70 \mathrm{HJ}$

\section{Second tour d'extirpation et suivants}

À la demande.

\section{ID Opérations à réaliser}

Période favorable : saison humide (éviter la saison sèche)

Rabattre les touffes à la machette à 20-30 cm au-dessus du sol.

\section{Un mois après}

Extirper les racines à la daba (Figure 28.2).

Sortir les plants de la parcelle (Figure 28.3).

Les mettre en tas (Figure 28.4).

Après séchage, brûler les tas, sous surveillance constante (Figure 28.5).

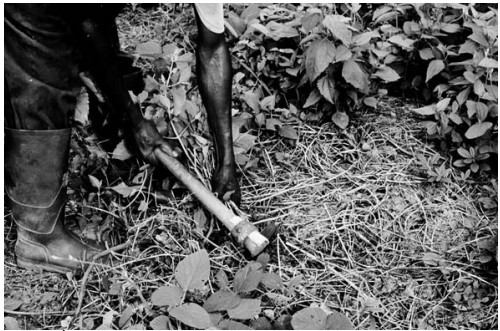

Figure 28.2.

Extirpation des racines de

Chromolaena odorata à la houe.

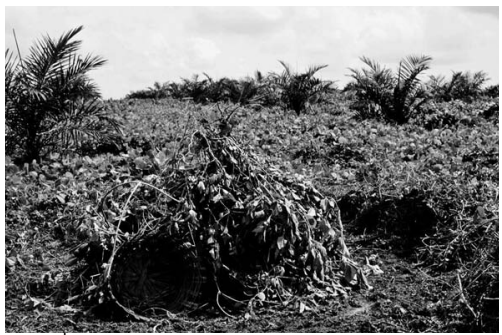

Figure 28.4.

Mise en tas des plants

de Chromolaena odorata.

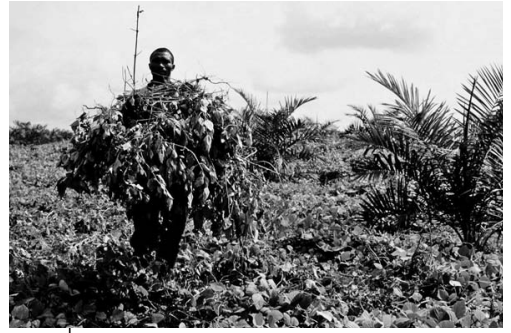

Figure 28.3.

Sortie des plants de Chromolaena sp.

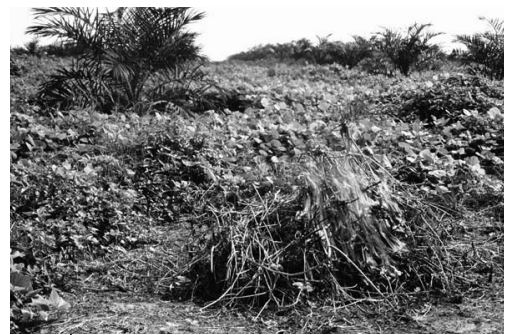

Figure 28.5.

Brûlage des souches. 


\section{Trois mois après}

Extirper les repousses à la daba ou à la main.

Sortir les repousses de la parcelle.

Les mettre en tas.

Après séchage, brûler les tas, sous surveillance constante.

\section{Attention}

Si l'ensemble de la parcelle de 5 hectares est envahie, il vaut mieux rabattre 1 hectare par mois, et donc n'avoir qu'1 hectare à extirper le mois suivant plutôt que de rabattre les 5 hectares en une fois car la charge de travail pour l'extirpation sera trop grande.

$\mathrm{Ne}$ pas laisser la parcelle se salir après une extirpation complète. Profiter des tours de rabattage pour extirper à la daba ou à la main, toute souche ou toute repousse de Chromolaena qui aurait échappé au traitement. Plus tard, les palmiers produiront plus et seront plus faciles à récolter.

Ne pas laisser les tas secs brûler sans surveillance car le risque de propagation du feu vers la plante de couverture ou les parcelles environnantes est très élevé. 


\section{Fiche 29. Cultures intercalaires et élevage}

On appelle cultures intercalaires, les cultures vivrières annuelles ou bisannuelles installées entre les jeunes palmiers plantés. Elles sont souvent mises en place par l'exploitant soit pour subvenir à ses besoins alimentaires soit comme source complémentaire de revenu pendant la période improductive du palmier.

Les travaux agricoles sont le plus souvent conduits manuellement. Aussi, un homme seul ne peut réaliser conjointement à ses 5 hectares de palmeraie plus de 1 hectare de cultures vivrières intercalaires. Au-delà, il sera amené à faire appel à de la main-d'œuvre extérieure.

Le choix des cultures dépend des habitudes alimentaires et des marchés potentiels. Leur mise en place et leur conduite doivent respecter les principes suivants :

- pas de brûlage après la plantation des palmiers. Maintien d'une zone sans culture de $3 \mathrm{~m}$ de diamètre en année 1 et année 2 ;

- ne jamais couper de feuilles aux jeunes palmiers pour laisser plus de place aux cultures vivrières. Maintien d'une zone vide de culture de 1,5 m au-delà de l'aplomb des feuilles à partir de l'année 3, incluse ;

- faire en sorte que toutes les cultures vivrières soient arrêtées à la mise en récolte des palmiers.

\section{Attention}

Certaines cultures répétées épuisent le sol, comme le manioc.

Certaines cultures encombrantes gênent les jeunes palmiers lorsqu'elles sont trop proches (manioc, plantain, maïs).

Les cultures vivrières associées attirent de nombreux ravageurs dont les rongeurs.

La surveillance sanitaire des palmiers doit être accrue.

Les parcelles de palmiers peuvent sous certaines conditions être utilisées dans les circuits de pacage. Cependant, si le nombre d'animaux (petit ou gros bétail), est trop élevé, il y a risque de dégradation de la productivité des palmiers.

Si des animaux paissent sur des parcelles en jeunes cultures sous couverture de légumineuse, il faut veiller à laisser le temps à la plante de couverture de se régénérer entre deux passages.

Les animaux ne doivent pas être laissés sans surveillance au moins tant que les extrémités de feuilles sont accessibles aux animaux, sinon des dégâts importants aux feuilles peuvent être occasionnés (figure 29.1). 


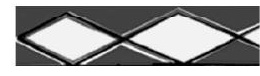

Ceux-ci entraineront immanquablement des retards de croissance et des réductions de production.

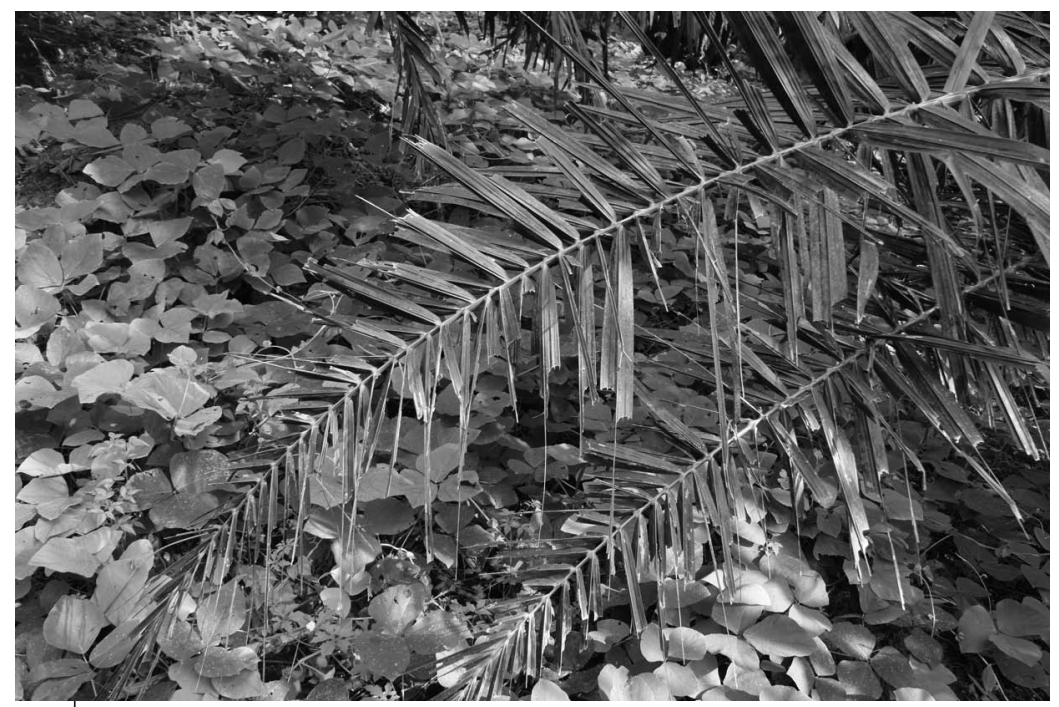

Figure 29.1.

Dégâts aux feuilles provoqués par une vache. 


\section{Fiche 30. Surveillance phytosanitaire}

La surveillance phytosanitaire est indispensable pour déceler à temps les différents dégâts de ravageurs et les maladies pouvant affecter les jeunes palmiers.

Il faut donc :

- contrôler tous les palmiers plantés une fois par mois;

- noter ou faire noter les observations dans un cahier.

Porter attention plus particulièrement aux grillages : jusqu'en fin de l'année 1, remettre en place les grillages déplacés.

Examiner plus particulièrement :

- la base des feuilles et du pseudobulbe (attaques de rats, d'agoutis et de vers blancs);

- les aisselles des feuilles et flèches (attaques d'Oryctes ou gros scarabée noir et chenilles de pyrales);

- les folioles (attaques d'insectes défoliateurs, d'oiseaux tisserins, d'acariens, de cercosporiose, de fusariose, etc.)

Si des attaques sont observées :

- se reporter au chapitre 7 «Défense des cultures», ou au livre «Le Palmier à huile» ou faire appel au conseiller agricole le plus proche; - contrôler tous les arbres une fois par semaine et noter la présence de rongeurs, de vers blancs et d'Oryctes. 


\section{Fiche 31. Fertilisation}

Une bonne fertilisation des jeunes palmiers est un gage de leur bon développement et d'une bonne entrée en production. Les types d'engrais et quantités proposées sont valables pour des conditions moyennes. Elles peuvent être adaptées en fonction des conditions locales avec l'aide du conseiller agricole.

\section{Engrais et quantités conseillées}

Tableau 31.1. Engrais et quantités (en g par arbre) à apporter.

\begin{tabular}{lccc}
\hline Années & $\begin{array}{c}\text { Urée } \\
\text { (g par arbre) }\end{array}$ & $\begin{array}{c}\text { Kiesérite } \\
\text { (g par arbre) }\end{array}$ & $\begin{array}{c}\text { Chlorure de potasse } \\
\text { (g par arbre) }\end{array}$ \\
\hline Année 1 & $2 \times 100 \mathrm{~g}$ & $2 \times 50 \mathrm{~g}$ & $2 \times 100 \mathrm{~g}$ \\
\hline Année 2 & $2 \times 200 \mathrm{~g}$ & - & $1 \times 200 \mathrm{~g}$ \\
\hline Année 3 & $2 \times 200 \mathrm{~g}$ & - & - \\
\hline
\end{tabular}

Le premier épandage se fait en juillet, le second en avril.

Tableau 31.2. Quantités d'engrais nécessaires pour 5 ha.

\begin{tabular}{lcccc}
\hline \multirow{2}{*}{ Année } & $\begin{array}{c}\text { Mois de } \\
\text { l'application }\end{array}$ & $\begin{array}{c}\text { Urée } \\
(\mathbf{k g})\end{array}$ & $\begin{array}{c}\text { Kiesérite } \\
(\mathbf{k g})\end{array}$ & $\begin{array}{c}\text { Chlorure de potasse } \\
(\mathbf{k g})\end{array}$ \\
\hline \multirow{2}{*}{ Année 1 } & Juillet & 72 & 36 & 72 \\
\cline { 2 - 5 } & Avril & 72 & 36 & 72 \\
\hline \multirow{2}{*}{ Année 2 } & Juillet & 144 & - & 144 \\
\cline { 2 - 5 } & Avril & 144 & - & - \\
\hline \multirow{2}{*}{ Année 3 } & Juillet & 144 & - & - \\
\cline { 2 - 5 } & Avril & 144 & - & \\
\hline
\end{tabular}

Fabrication d'une mesure pour $50 \mathrm{~g}$ d'engrais :

- se munir d'une boîte vide de lait concentré ou de concentré de tomate de 400 à $500 \mathrm{~g}$ de contenance ou une bouteille d'eau en plastique (comme sur la photo);

- découper le couvercle;

- peser $50 \mathrm{~g}$ d'engrais sur une balance précise au gramme près (chez un commerçant ou chez un pharmacien);

- verser l'engrais dans la boîte;

- bien niveler l'engrais sans le tasser;

- découper la boîte à la hauteur du niveau de l'engrais; 
- inscrire le chiffre 50 sur le fond de la boîte, à la peinture ou en gravant les chiffres avec un clou.

Recommencer la procédure pour fabriquer une mesure de 100 puis de 200 g. Ces mesures sont utilisables quel que soit le type d'engrais utilisé.

\section{ID Application de l'engrais}

Se munir d'un seau plastique propre et sec et de la mesure adaptée à la quantité d'engrais à apporter par arbre (figure 31.1).

Déposer les sacs d'engrais au bord de la parcelle.

Découdre soigneusement l'ouverture d'un sac.

Remplir le seau aux 3/4 avec l'engrais choisi.

Se placer dans le rond du jeune palmier.

Remplir la mesure à ras bord sans faire bomber le produit (figure 31.2).

Déposer l'engrais en large couronne autour du palmier à $50 \mathrm{~cm}$ minimum du pseudobulbe (figure 31.3).

\section{Attention}

Conserver l'engrais sur une claie, à l'abri de l'eau et de la lumière.

Ne jamais mélanger un reste d'engrais à un autre reste d'engrais de nature différente.

Bien refermer les sacs entamés avec une ficelle solide.

Peser les sacs entamés avant d'acheter l'engrais pour l'épandage suivant.

Faire l'épandage après un tour d'entretien de la parcelle (couverture et ronds).

Ne pas faire d'épandage d'engrais sous la pluie.

Ne pas faire d'épandage d'urée en pleine saison sèche.

Attendre une semaine entre l'épandage de l'urée et celui des autres engrais.

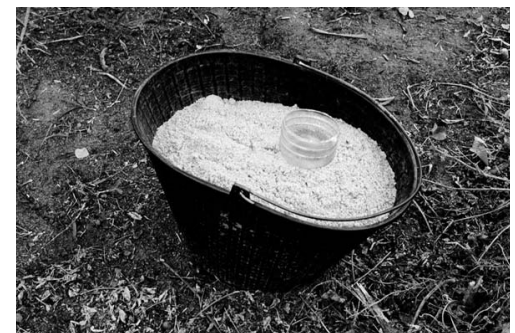

Figure 31.1.

Seau nécessaire

à la fertilisation.

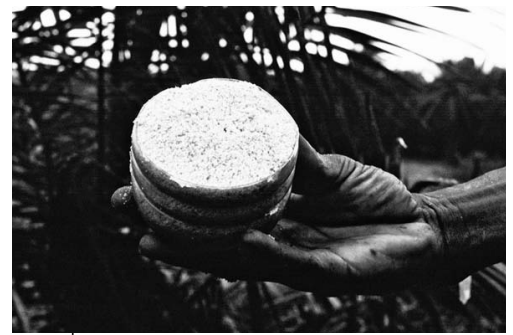

Figure 31.2.

Exemple de mesure correctement remplie. 

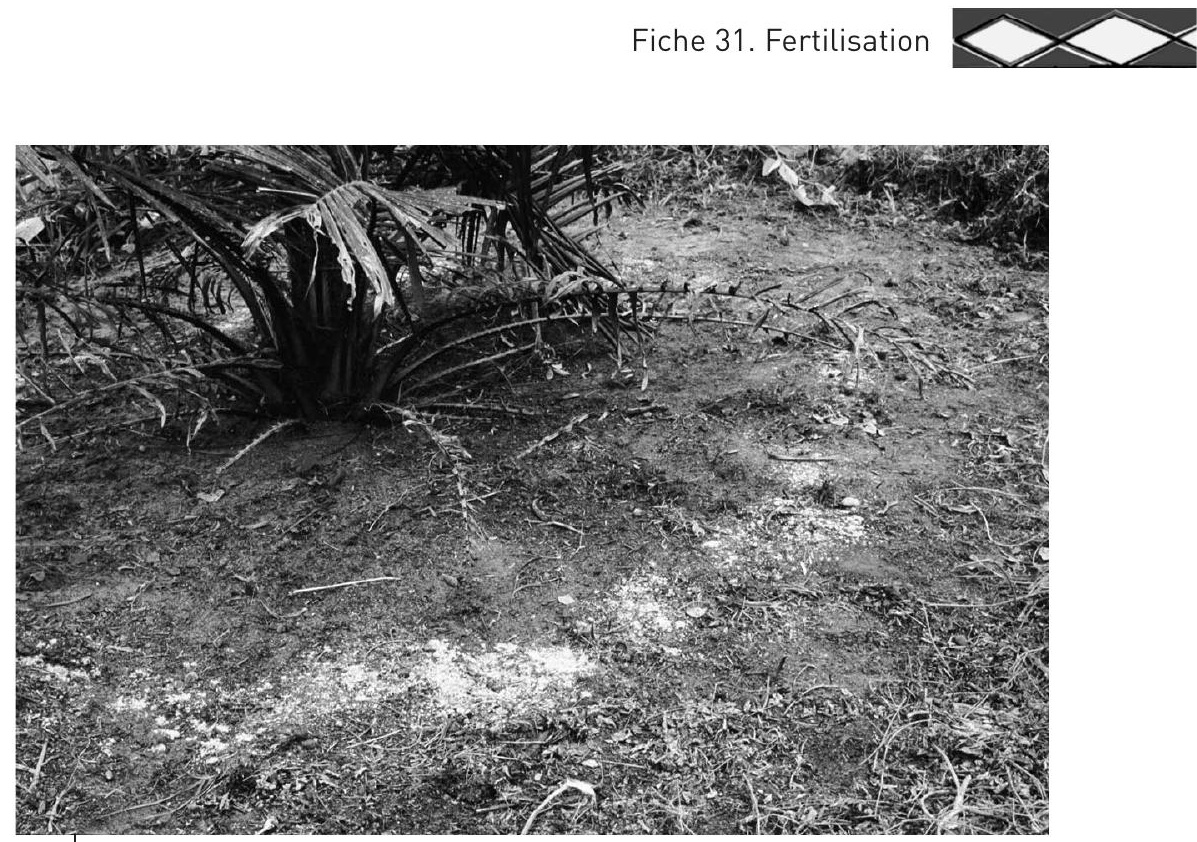

Figure 31.3.

Application de l'engrais en couronne. 



\section{Palmiers en exploitation}

La phase productive du palmier dure de 20 à 30 ans. Pour exprimer tout son potentiel, la plantation doit être correctement fumée et entretenue, les ronds bien dégagés. Les feuilles sèches et surnuméraires doivent être enlevées de la couronne de façon à permettre un accès aisé aux régimes.

La qualité de la récolte est un atout important car les acheteurs de régimes y sont très sensibles.

Enfin, les différents outils de récolte utilisables sont brièvement décrits ainsi que leur mode d'utilisation. 


\section{Fiche 32. Entretien des ronds}

\section{IID Objectif}

Permettre de détecter les régimes mûrs et faciliter le ramassage des fruits détachés.

\section{ID Besoins en matériel}

- 1 machette légère

- 1 gabarit

- Bottes ou chaussures de sécurité.

\section{ID Temps de travaux}

- $1 \mathrm{HJ} / \mathrm{ha} \times 5 \mathrm{ha}=5 \mathrm{HJ} \times 4$ passages $/$ an $=20 \mathrm{HJ}$.

\section{ID Technique}

Utiliser une machette légère.

Se protéger les jambes en utilisant un bâton tenu en main de façon à couper la trajectoire de la machette.

Fabriquer un gabarit avec un bois ou un bambou fin de 1,5 $\mathrm{m}$ de long. Nettoyer, jusqu'à obtenir le sol nu, un rond de rayon égal au gabarit tout autour du palmier (figures 32.1 et 32.2).

Enlever les tiges de plantes grimpantes qui ont pu envahir le palmier. Vérifier la dimension du rond avec le gabarit.

Procéder palmier par palmier, puis ligne par ligne.

Faire un tour de nettoyage des ronds tous les 3 mois environ en fonction de l'état de leur envahissement par les plantes adventices :

- prévoir un tour de nettoyage des ronds juste avant la mise en récolte;

- prévoir un tour de nettoyage des ronds juste avant le début de la pointe de production. 


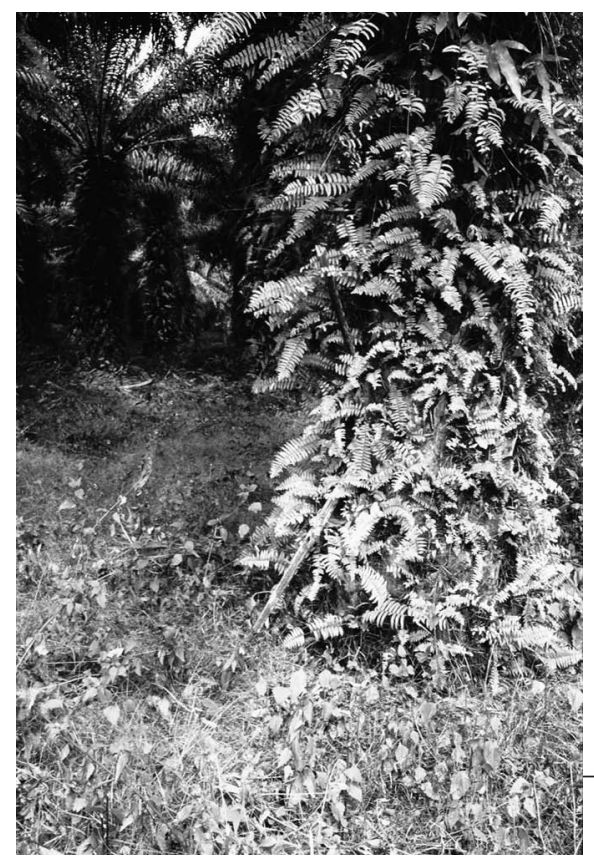

Figure 32.1.

Rond avant sarclage.

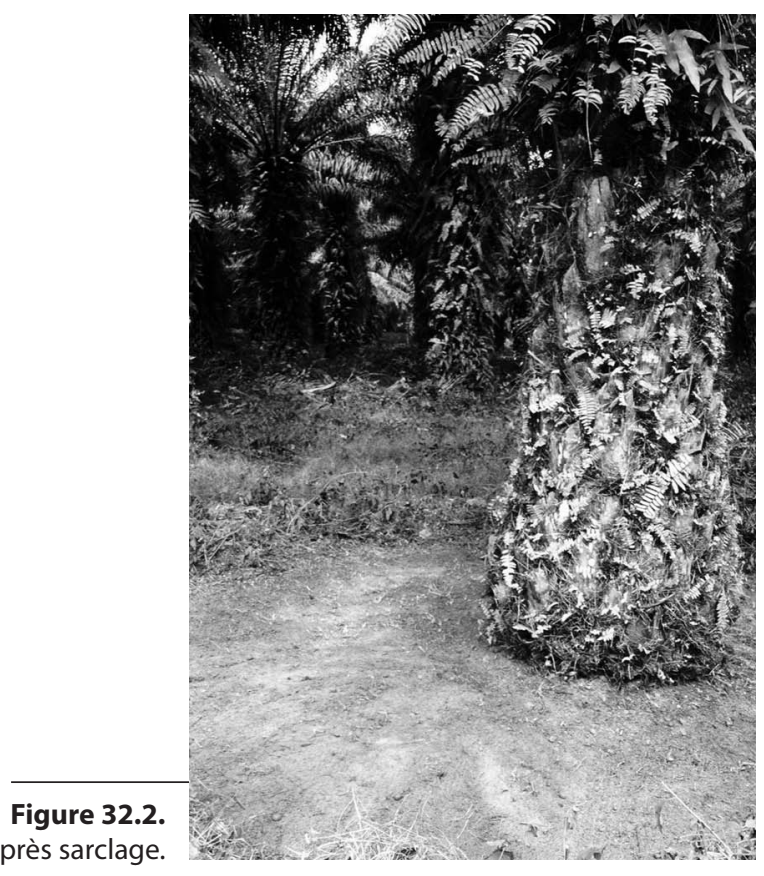

Rond après sarclage. 


\section{Fiche 33. Entretien des interlignes}

\section{IID Objectif}

Faciliter la récolte et la surveillance de la plantation. Éviter trop de concurrence pour l'eau, la lumière et les éléments minéraux (figure 33.1).

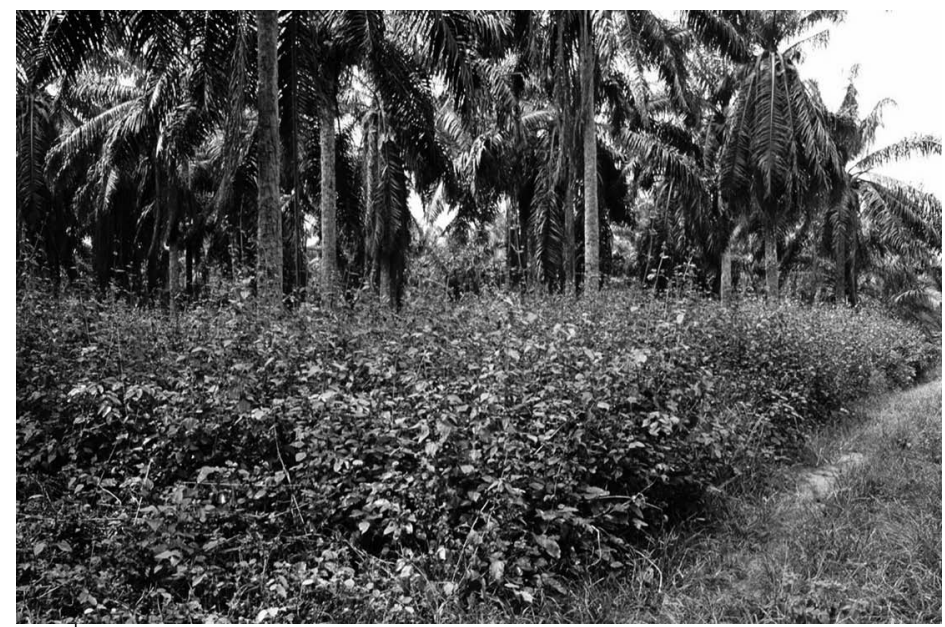

Figure 33.1.

Interligne avant entretien : les arbres à l'arrière-plan font partie de la plantation et le Chromolaena mesurait à cet endroit au moins $2 \mathrm{~m}$ de haut.

\section{I) Besoins en matériel}

- 1 machette lourde

- Bottes ou chaussures de sécurité.

ID Temps de travaux

- $1 \mathrm{HJ} /$ ha $\times 5$ ha $=5 \mathrm{HJ} \times 3$ passages $/$ an $=15 \mathrm{HJ}$.

\section{II) Technique}

Utiliser une machette lourde.

Se protéger les jambes en utilisant un bâton tenu en main de façon à couper la trajectoire de la machette.

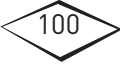


Couper les repousses d'arbres, d'arbustes et de végétaux buissonnants (à l'exception des plantes envahissantes comme le Chromolaena odorata) à environ $1 \mathrm{~m}$ de hauteur (figure 33.2).

Faucher les graminées et les plantes envahissantes le plus bas possible $(20 \mathrm{~cm})$ (figure 33.3).

Procéder interligne après interligne.

Faire un passage d'entretien tous les 4 mois en fonction de l'état moyen des interlignes :

- prévoir un passage d'entretien juste avant la mise en récolte;

- prévoir un passage d'entretien juste avant la pointe de production.

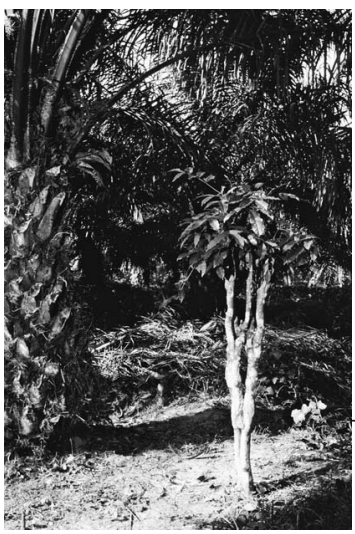

Figure 33.2.

Bonne hauteur de rabattage des ligneux et arbustes.

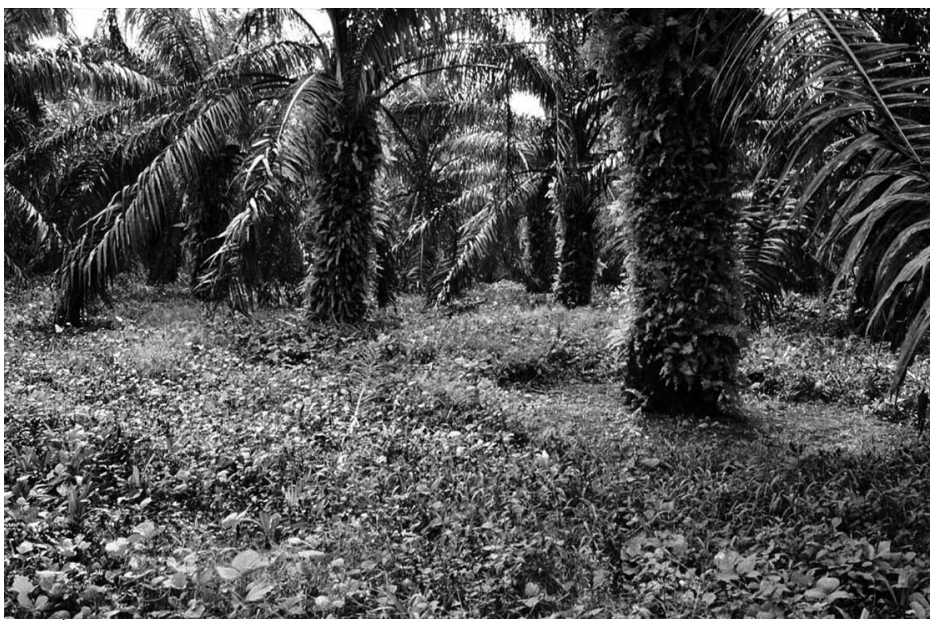

Figure 33.3.

Interligne après entretien.

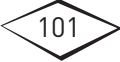




\section{Fiche 34. Entrée en production}

\section{IID Objectif}

Préparer les jeunes arbres pour leur première récolte. Les travaux décrits ci-dessous sont à réaliser dans le mois qui précède la mise en récolte (figures 34.1 et 34.2).

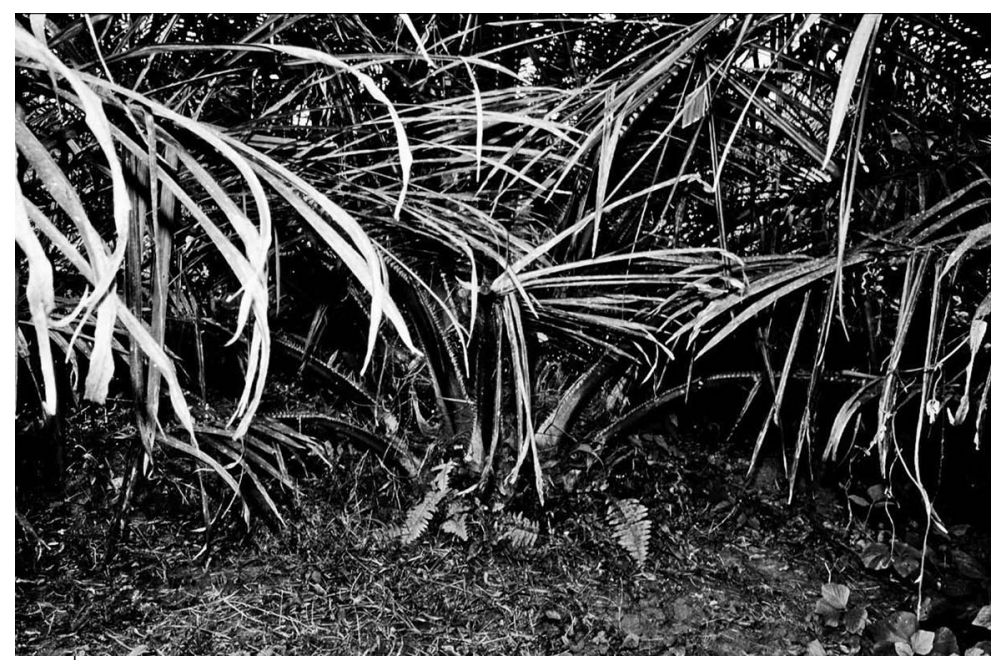

Figure 34.1.

Palmier avant préparation de la couronne.

\section{ID Besoins en matériel}

- 1 machette lourde

- 1 ciseau de récolte

- 1 paire de gants épais

- Bottes ou chaussures de sécurité.

ID Temps de travaux

- $1,5 \mathrm{HJ} / \mathrm{ha} \times 5$ ha $=7,5 \mathrm{HJ}$.

\section{II) Technique}

Ne pas oublier de faire au préalable, un tour d'entretien des interlignes.

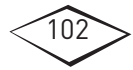


Utiliser une machette lourde et un ciseau de récolte.

Couper, bien à leur base, toutes les feuilles entièrement desséchées.

Tronçonner les feuilles en deux, partie épineuse (le pétiole) d'un côté et le reste de l'autre (la palme).

Disposer les pétioles, bien rangés, épines vers le bas, sur l'andain.

Disposer les palmes entre deux palmiers le plus près possible de l'andain et perpendiculairement à la ligne de plantation.

Arracher les inflorescences mâles sèches.

Les jeter sur les tas de feuilles.

Avec le ciseau, couper tous les régimes pourris ou mal formés.

Jeter ces régimes sur les tas de pétioles.

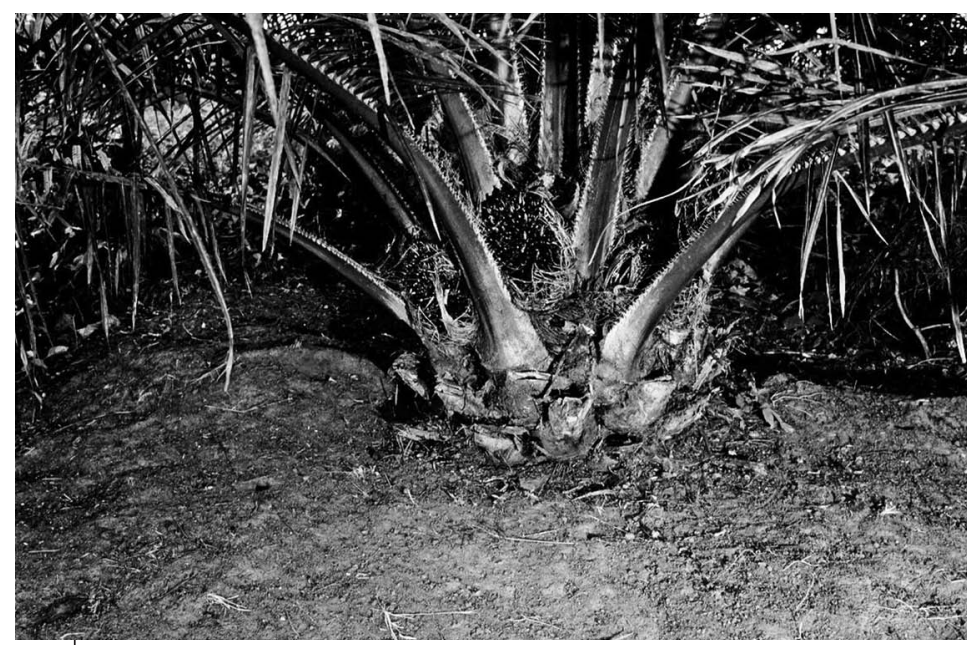

Figure 34.2.

Palmier après préparation de la couronne. 


\section{Fiche 35. Élagage}

\section{IID Objectif}

Maintenir les couronnes propres pour bien observer la maturité des régimes et faciliter leur récolte (figures 35.1 et 35.2).

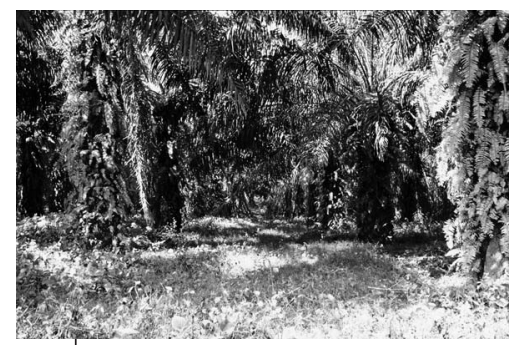

Figure 35.1.

Palmiers avant élagage.

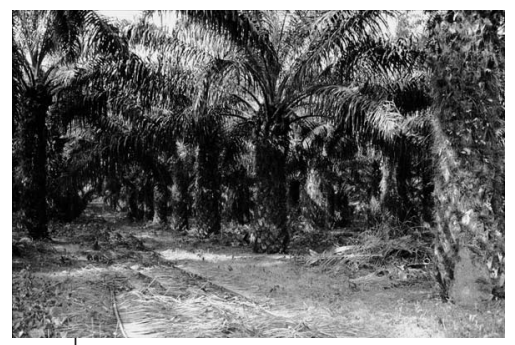

Figure 35.2.

Palmiers après élagage.

\section{Besoins en matériel}

Tableau 35.1. Outils à utiliser.

\begin{tabular}{lc}
\hline Mode de récolte & Outil d'élagage \\
\hline Ciseau & Machette \\
\hline Machette & Machette \\
\hline Faucille & Faucille \\
\hline Ceinture & Machette ou hache \\
\hline
\end{tabular}

- Équipement de sécurité

- Gants épais, bottes ou chaussures de sécurité, casque de chantier dès que les palmes à couper sont à plus de 2,5 m de hauteur.

\section{II) Critères}

Jusqu'à la fin de l'année 5 : ne couper que les feuilles entièrement sèches.

Du début de l'année 6 à la fin de l'année 15 : laisser 2 feuilles sous le régime mûr ou 5 feuilles par spire.

Du début de l'année 16 jusqu'à la fin de l'exploitation : laisser 1 feuille sous le régime mûr.

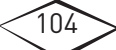




\section{II) Périodicité}

Le premier tour d'élagage se fait avant la pointe de production, de préférence avant le tour de nettoyage des ronds.

Le second tour d'élagage se fait de 6 à 9 mois après, selon l'état des couronnes.

\section{ID Pratique}

Revêtir les équipements de sécurité.

Couper les feuilles au plus près du stipe.

Couper les feuilles en deux : la partie épineuse (le pétiole) d'un côté et la palme de l'autre (rachis portant les folioles).

Disposer les parties épineuses entre 2 lignes de palmiers (l'andain), le plus loin possible des ronds (figure 35.3).

Étaler grossièrement les palmes dans l'interligne libre dans le sens des lignes et entre deux palmiers perpendiculairement à la ligne de plantation. Cette mesure permet de lutter contre l'érosion et répartit mieux la matière organique (figure 35.4).

\section{ID Temps de travaux}

Variable selon l'état des couronnes.

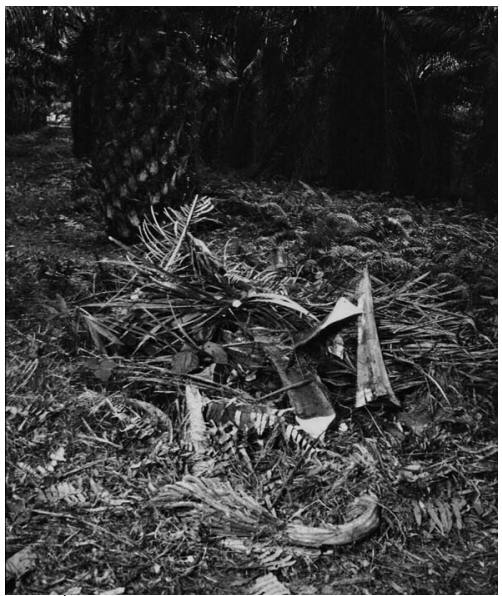

Figure 35.3.

Rangement des pétioles.

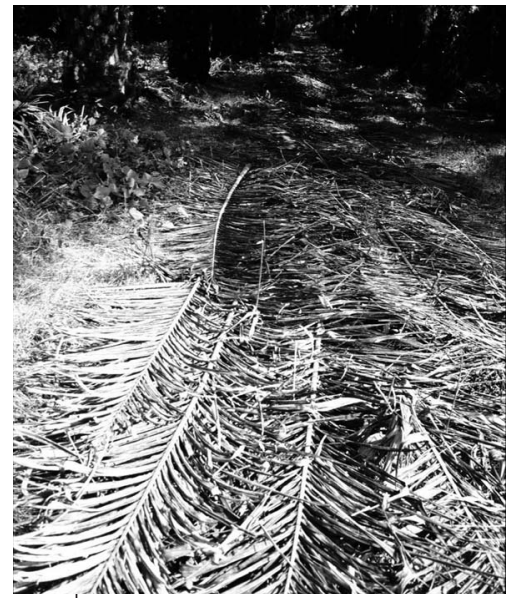

Figure 35.4.

Rangement des palmes dans l'interligne. 


\section{Fiche 36. Récolte des régimes}

Dans les conditions agroclimatiques normales, les arbres produisent des régimes tout au long de l'année. La production d'une parcelle est donc continue toute l'année. À chaque tour de récolte, il faut visiter tous les arbres. Néanmoins, il y a toujours des périodes de plus forte et de plus faible production.

\section{Matériel nécessaire}

- 1 machette lourde

- Outil de récolte ad-hoc

- 1 lime

- 1 pierre à aiguiser

- 1 brouette

- 1 petit balai en nervures de folioles

- 1 petit râteau

- Sacs vides pour les fruits détachés

- Gants de protection

- Bottes ou chaussures de protection

- Casque de chantier, dès que le régime est à plus de $2,5 \mathrm{~m}$ de hauteur.

Temps de travaux

- Environ 3 à $4 \mathrm{HJ}$ par tonne de régime.

\section{ID Périodicité}

De 2 à 4 tours par mois. La fréquence des tours de récolte est normalement déterminée par celle de la collecte des régimes bordchamp. Celle-ci est souvent effectuée par la plantation industrielle voisine ou par un transporteur intermédiaire.

La coupe, le transport des régimes et des fruits détachés jusqu'à l'aire de stockage bord-champ doivent être effectués pendant les 2 ou 3 jours au maximum qui précèdent le passage du véhicule collecteur.

\section{ID Critères de maturité}

Régime mûr. $\mathrm{Au}$ minimum 2 fruits détachés naturellement dans le rond et au moins 10 fruits détachés après la coupe (figure 36.1). 
Régime vert. Tout régime dont la maturité n'atteint pas le seuil ci-dessus.

Régime pourri. Tout régime dont le pédoncule est partiellement ou entièrement nécrosé ou pourri (figure 36.2).

Sur les arbres de moins de 5 ans, il est préférable de couper le régime dès que 2 fruits mûrs sont détachables aisément.

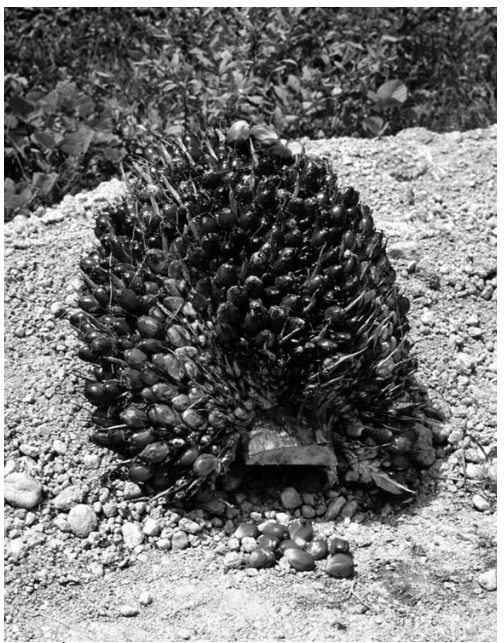

Figure 36.1.

Exemple de régime mûr.

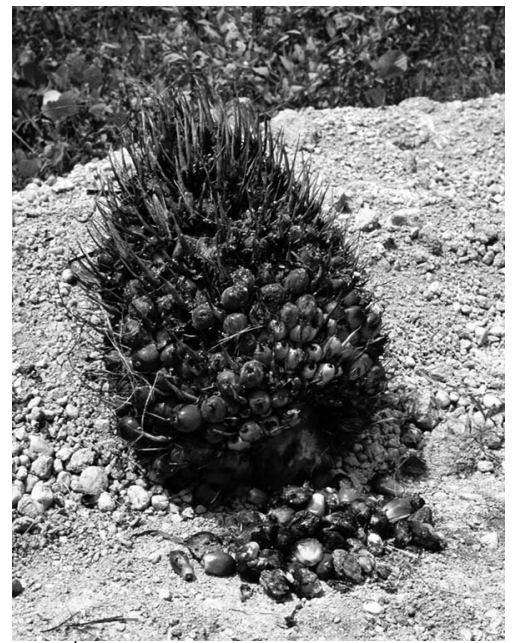

Figure 36.2.

Exemple de régime pourri.

\section{II) Opérations}

\section{Préalable}

Revêtir les équipements de sécurité : gants, chaussures ou bottes, casque de chantier le cas échéant.

\section{Séquence 1 : coupe du régime et rassemblement des fruits}

Vérifier la présence et le nombre de fruits détachés dans le rond.

Se placer du côté où le pédoncule du régime est le plus dégagé.

Couper complètement les feuilles situées sous le régime, sauf dans le cas de la récolte au ciseau.

Couper le pédoncule du régime.

Faire tomber les fruits restés bloqués dans la couronne ou derrière les chicots du stipe. 
Raccourcir le pédoncule du régime au ras de sa base.

Découper les feuilles et les ranger (voir Fiche 35 - Élagage).

Mettre le régime bien en évidence dans le rond.

Débarrasser le régime des débris végétaux et de la terre avec un petit balai de fibres.

Rassembler les fruits détachés auprès du régime en prenant soin de ne pas les mélanger avec des débris végétaux ou du sable (figure 36.3). Il est possible d'utiliser un petit râteau à cet effet.

\section{Séquence 2 : transport du régime bord-champ}

Transporter les régimes et les fruits vers l'aire de stockage bord-champ avec la brouette.

Mettre les régimes en tas (figure 36.4).

Mettre les fruits détachés dans des sacs.

Abriter le tas de régimes des intempéries et du soleil avec des palmes.

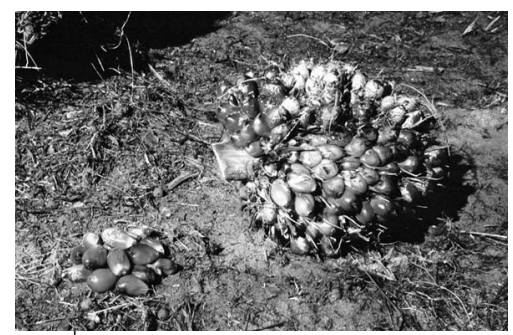

Figure 36.3.

Régime mûr et fruits détachés prêts à être transportés bord-champ.

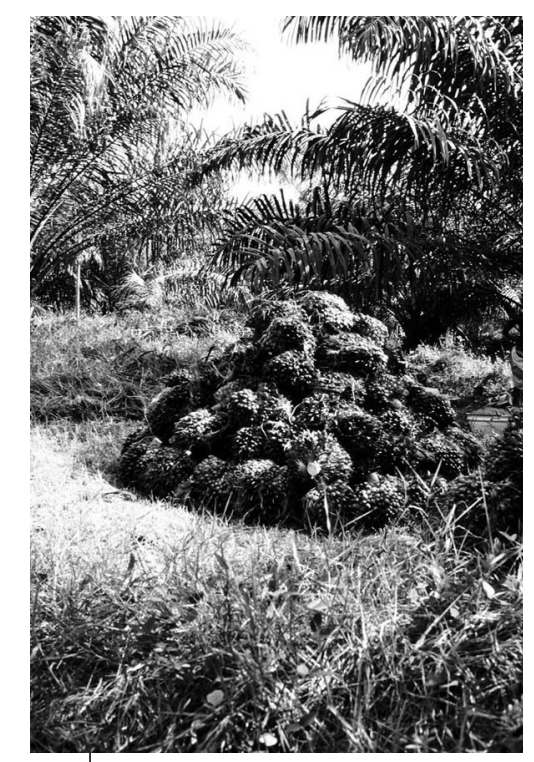

Figure 36.4.

Tas de régimes bord-champ.

Le récolteur peut être aidé par une tierce personne qui s'occupera de rassembler et collecter les fruits détachés et de transporter les régimes bord-champ. 


\section{Fiche 37. Techniques de récolte}

\section{ID Généralités}

Affûter à la lime pour diminuer l'épaisseur du métal (1 fois par jour au maximum).

Donner du fil au tranchant avec une pierre à affûter.

Éviter de planter la partie tranchante de l'outil de récolte dans le sol, pour ne pas détruire le fil.

Avant et après le travail, envelopper la partie tranchante de l'outil dans une toile solide ou un fourreau pour la protéger et éviter les accidents.

\section{Attention}

Un outil bien affûté coupe une feuille de papier sans effort.

Un outil bien tranchant épargne la fatigue, les blessures musculaires et articulaires.

Adresse et outil bien affûté valent mieux que la force brutale.

\section{ID Récolte au ciseau étroit}

\section{Description de l'outil}

Lame droite, rectangulaire affûtée sur un petit côté et dont l'autre forme un manchon dans le même axe (figure 37.1).

Longueur de la partie droite : 20 à $25 \mathrm{~cm}$.

Largeur de la partie droite : $7 \mathrm{~cm}$ environ.

Épaisseur de la lame : 4 à 5 mm environ.

Manche cylindrique en bois d'environ 1,5 m de longueur.

\section{Utilisation}

De l'entrée en récolte à la fin de l'année 5.

\section{Pratiquement}

Prendre le manche de l'outil à deux mains.

Se placer du côté où le pédoncule du régime est le plus dégagé (figure 37.2).

Les coups sont portés dans l'axe du manche.

Sectionner le pédoncule du régime par un ou deux coups secs.

Extraire le régime de l'aisselle de la feuille en le faisant basculer avec la lame de l'outil. 


\section{ID Récolte à la machette}

\section{Description de l'outil}

Lame droite, grossièrement triangulaire, plus large à son extrémité, munie d'une queue de fixation courte, percée de 2 ou 3 trous.

Affûtage sur le plus grand côté de la lame (figure 37.3).

Longueur de l'outil : 50 à $60 \mathrm{~cm}$.

Largeur de la lame : maximum $10 \mathrm{~cm}$.

Épaisseur de la lame : 3 mm environ.

Poignée de maintien appliquée sur la queue de fixation.

\section{Utilisation}

Du début de l'année 6 jusqu'à ce que la couronne ne soit plus aisément accessible (environ 1,8 $\mathrm{m}$ de hauteur).

\section{Pratiquement}

Prendre l'outil dans une main.

Faire sauter les épines des feuilles porteuses d'un coup de lame.

Soutenir les feuilles à couper de l'autre main. Il est recommandé de protéger cette main avec un gant solide.

Couper les feuilles à leur base, le plus près possible du stipe.

Faire une coupe légèrement en biais (figure 37.4).

Utiliser le dernier quart de la lame pour bien entailler les pétioles.

\section{ID Récolte à la faucille}

\section{Description de l'outil}

Lame courbe ouverte avec une queue de fixation munie d'un trou ou d'un talon. Cette lame est également appelée «couteau malais» (figure 37.5).

Longueur développée de la lame : $40 \mathrm{~cm}$ environ.

Largeur de la lame : 4 à $5 \mathrm{~cm}$.

Épaisseur de la lame : $3 \mathrm{~mm}$ environ.

Rayon de courbure : $15 \mathrm{~cm}$.

Longueur de la queue : $17 \mathrm{~cm}$.

Manche en bois, en bambou malais ou en tube d'aluminium. La longueur du manche est comprise entre 3 et $10 \mathrm{~m}$ selon la hauteur des arbres à récolter. Ce manche doit être assez souple, surtout au-delà de $6 \mathrm{~m}$. 


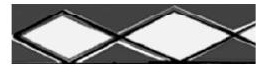

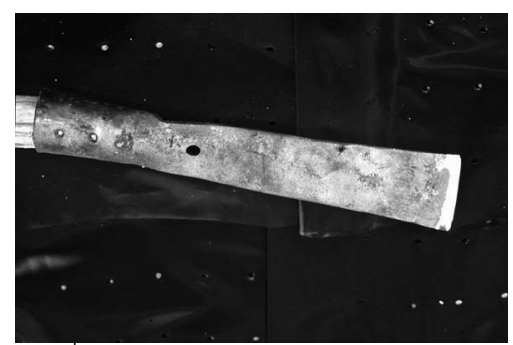

Figure 37.1.

Lame de ciseau étroit.

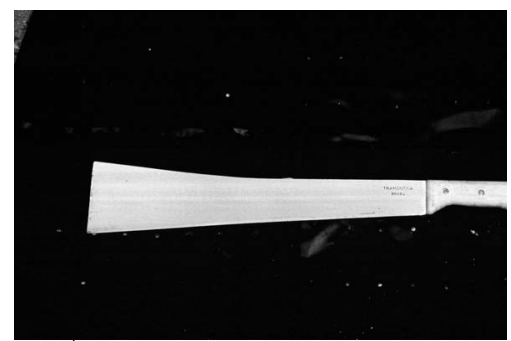

Figure 37.3.

Machette pour la récolte.

\section{Utilisation}

Lorsque la machette n'est plus utilisable.

\section{Pratiquement}

Prendre l'outil à deux mains vers l'extrémité du manche.

Se placer du côté où le pédoncule du régime est le plus dégagé.

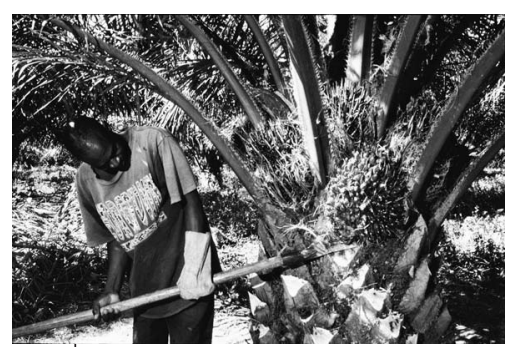

Figure 37.2.

Positionnement de la lame pour la coupe du régime.

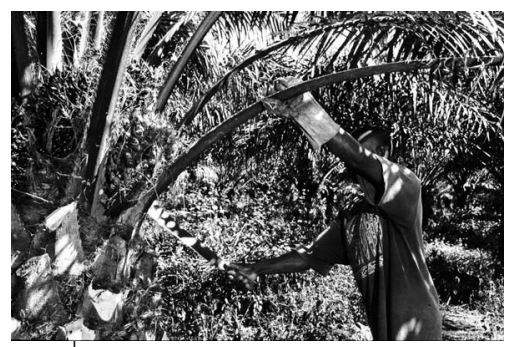

Figure 37.4.

Coupe à la machette.

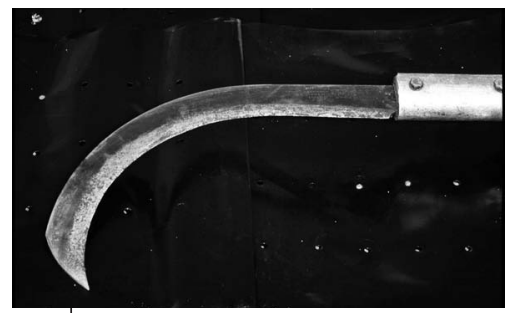

Figure 37.5.

Le couteau malais.

Lever l'outil en direction de la couronne.

Insérer la lame au-dessus des pétioles des feuilles ou derrière le pédoncule du régime à couper, légèrement en biais.

Lopérateur doit être placé à environ $2 \mathrm{~m}$ de la base du stipe, aussi la longueur du manche doit être bien adaptée à la hauteur de l'arbre (figures 37.6 à 37.8).

Tirer vers le bas dans l'axe du manche d'un coup sec et ample.

Bien faire glisser le tranchant de la lame sur la partie à couper. 
Exploiter la souplesse du manche pour accélérer le mouvement.

Reprendre jusqu'à ce que la feuille ou le pédoncule du régime soient coupés.

Après la coupe, ne pas laisser retomber l'outil, mais le caler en regard du prochain régime à couper sur un des arbres suivants.

Avec une lame parfaitement affûtée, un coup suffit pour trancher la base d'un pétiole ou le pédoncule d'un régime.

\section{Attention}

Une lame parfaitement affûtée réduit considérablement la fatigue du coupeur.

Un bon technicien ne s'épuise pas à relever sa faucille entre chaque arbre, mais se déplace en la tenant bien à la verticale.

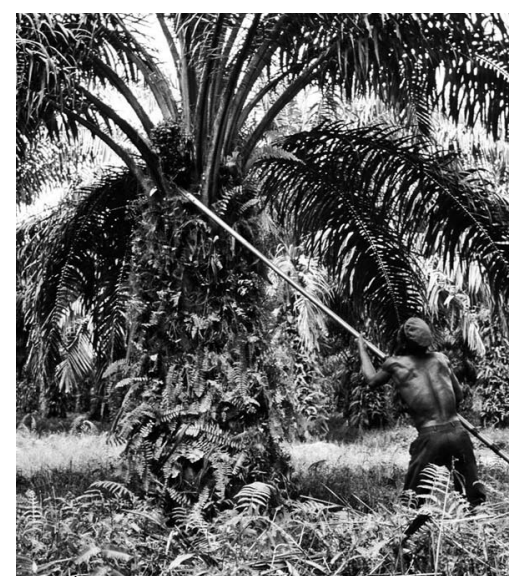

Figure 37.6.

Coupe du régime avec une faucille à manche court.

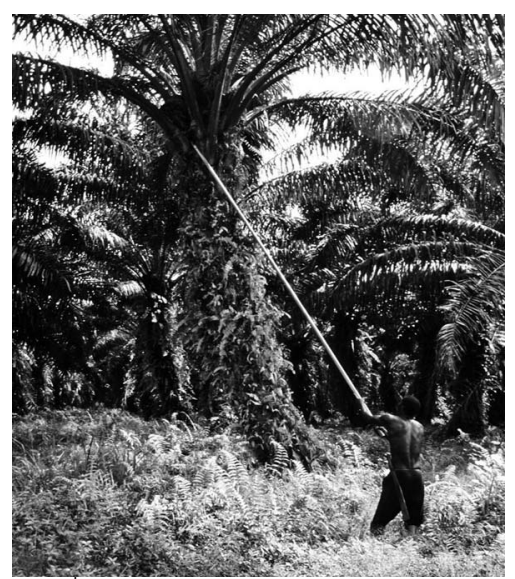

Figure 37.7.

Coupe du régime avec une faucille à manche moyen.

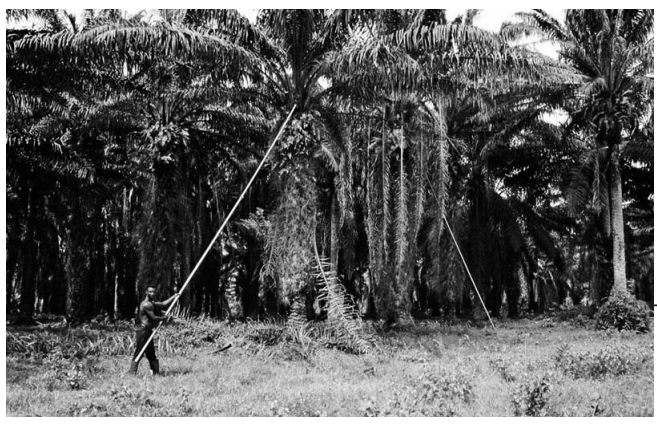

Figure 37.8.

Coupe du régime avec une faucille à manche long. 


\section{Fiche 38. Fumure}

\section{II) Généralités}

Les palmiers en exploitation doivent recevoir une fertilisation chaque année ou tous les deux ans pour compenser les pertes dues à l'exportation de la production. En l'absence de fumure, le potentiel de production du palmier s'affaiblira.

En plantation industrielle, les apports d'engrais sont fixés, par des spécialistes, après une étude approfondie de la situation nutritionnelle de la plantation et de la politique de fertilisation à mettre en place. Basée notamment sur des prélèvements et analyses de la composition chimique des feuilles, cette stratégie n'est pas à la portée du petit exploitant villageois.

Dès lors, deux possibilités lui sont offertes :

- appliquer la même politique de fumure que la plantation industrielle la plus proche (si elle suit les préconisations mentionnées dans l'ouvrage «Le palmier à huile» [Jacquemard, 2011]);

- avec le groupement auquel il appartient, demander à un organisme spécialisé (par exemple un Centre de recherche habilité) de faire un contrôle nutritionnel régulier sur tout ou partie des plantations villageoises du groupement. Le conseiller agricole formulera les recommandations nécessaires.

Des mesures simples permettent d'économiser l'engrais :

- s'interdire d'exporter les résidus de feuilles coupées pour la récolte ou l'élagage : folioles, rachis ou pétioles;

- passer un contrat avec l'huilerie pour récupérer les rafles des régimes qui ont été traités ou si cela n'est pas possible, leur équivalent en engrais potassique;

- ne pas appliquer d'engrais sous la pluie ou après une forte pluie;

- ne pas appliquer d'urée en saison sèche;

- appliquer l'engrais sur l'andain et les feuilles disposées entre les palmiers et non dans le rond.

\section{IID Pratique de la fumure}

\section{Dates d'application}

De préférence en deux fois, la première juste au retour des pluies en mars-avril, la seconde en saison peu pluvieuse, en août-septembre. 


\section{Mode d'application}

En large couronne, sur les débris végétaux entre les palmiers et sur l'andain.

\section{Technique d'application}

Exemple : application de $1000 \mathrm{~g}$ de chlorure de potassium $(\mathrm{KCl})$.

\section{Comment fabriquer une mesure pour $1000 \mathrm{~g}$ d'engrais ?}

Se munir d'une boîte vide de lait concentré, de concentré de tomate ou une boite de $1 \mathrm{~kg}$ de contenance.

Découper le couvercle.

Peser $1000 \mathrm{~g}$ d'engrais sur une balance précise à 10 grammes près chez un pharmacien ou un commerçant.

Déposer l'engrais dans la boîte.

Bien niveler l'engrais sans le tasser.

Couper la boîte au niveau de l'engrais.

Inscrire le chiffre 1000 sur le fond de la boîte à la peinture ou en gravant les chiffres avec un clou.

\section{Comment appliquer l'engrais ?}

Déposer les sacs d'engrais au bord de la parcelle au nord et au sud des lignes.

Se munir d'un seau, d'une bassine en plastique de 10 litres ou d'un ancien sac d'engrais et de la mesure.

Se protéger les mains avec des gants adaptés : des gants en caoutchouc doublés en coton (voir figure 44.2).

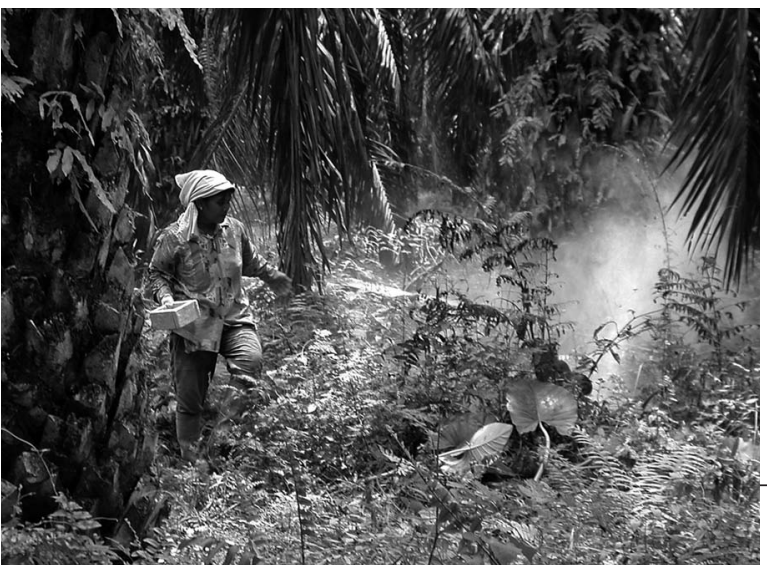

Figure 38.1.

Épandage en cours.

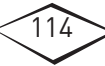


Se protéger la bouche et le nez avec un masque à poussière.

Porter un tablier en toile forte.

Découdre soigneusement l'ouverture d'un sac.

Remplir le seau au 4/5 avec l'engrais.

Se placer dans le rond du palmier.

Remplir la mesure à ras bord sans faire bomber le produit.

Projeter l'engrais avec la main en large couronne à l'extérieur du rond (figures 38.1 et 38.2) jusqu'à l'épuisement du contenu de la boîte.

Passer au palmier suivant.

Reprendre à partir de «Se protéger la bouche et le nez...» pour achever l'épandage de l'engrais.

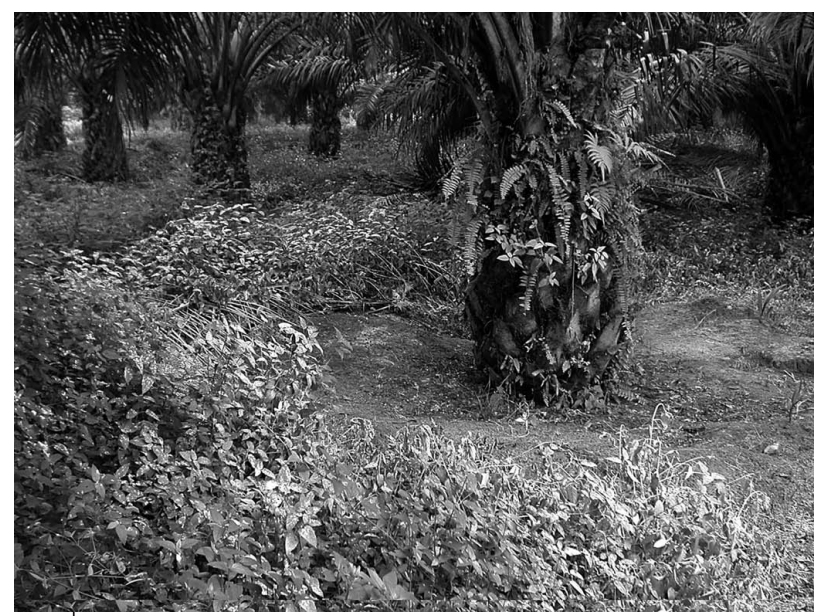

Figure 38.2.

Localisation de l'épandage.

\section{Attention}

Conserver l'engrais sur une claie, à l'abri de l'eau et de la lumière.

Ne jamais mélanger un reste d'engrais à un autre reste d'engrais de nature différente.

Bien refermer les sacs entamés avec une ficelle solide.

Peser les sacs entamés avant d'acheter les engrais pour l'épandage suivant afin de n'acheter que le nécessaire.

Faire l'épandage après un tour d'entretien de la parcelle (couverture et ronds). 


\section{Fiche 39. Épandage de rafles}

\section{Dénéralités}

Comme dans la fiche 38 «Fumure», l'application de rafles, c'est-àdire ce qui reste du régime après son traitement en huilerie, est un excellent complément dans un programme de fertilisation. Il apporte non seulement du potassium, mais aussi de la matière organique essentielle à la préservation de la fertilité de la parcelle.

Lhuilerie qui traite les régimes de palme des petits planteurs est le plus souvent intégrée dans un ensemble agro-industriel. Les rafles des régimes collectés chez les petits planteurs sont le plus souvent recyclées pour la plantation commerciale.

Le petit planteur est tout à fait en droit de négocier avec l'usine le retour des rafles de ses régimes. Pour ce retour, deux possibilités s'offrent :

- sous forme de compost;

- sous forme de rafles.

Ces rafles représentent 20 à $25 \%$ du poids des régimes exportés. Une tonne de rafles fraîches apporte environ l'équivalent de $6 \mathrm{~kg}$ de chlorure de potasse. Elle représente une économie sensible dans les achats d'engrais.

Il n'est pas possible de totalement substituer la fertilisation minérale avec ces rafles. Mais le petit planteur peut négocier un retour de ses rafles tous les 3-4 ans.

\section{Matériel et équipements}

- 1 paire de bottes

- 1 paire de gants

- 1 brouette de chantier

- 1 crochet métallique muni d'une poignée en «T».

\section{ID Comment organiser le travail ?}

Les rafles sont toujours déposées bord-champ par les camions de l'usine. Il faut savoir que les rafles perdent l'essentiel de leurs qualités nutritives au bout de 10 jours. Aussi, le planteur doit-il être capable d'épandre ses rafles dans les 2 jours qui suivent leur arrivée bordchamp. Il y a donc un programme de transport précis à établir avec 
les services techniques de l'usine. Le planteur, de son côté, doit programmer son travail en conséquence.

\section{II) Où appliquer les rafles?}

Lidéal est de disposer les rafles en couche continue d'une seule épaisseur, dans une placette rectangulaire de $2 \times 3 \mathrm{~m}$ sur la ligne entre deux palmiers (figure 39.1).

\section{IID Comment procéder?}

Revêtir les équipements de protection adéquats : bottes et gants.

Se munir d'un crochet métallique pour attraper et disposer les rafles et d'une brouette de chantier.

Charger les rafles avec le crochet dans la brouette.

Toujours commencer par les palmiers du milieu des lignes, puis continuer vers la tête de l'interligne en alternant une placette à droite et une placette à gauche.

Décharger les rafles au milieu entre deux palmiers et les mettre en place.

Compter le nombre de brouettées nécessaires pour couvrir le rectangle de $2 \mathrm{~m}$ x $3 \mathrm{~m}$.

Compléter si nécessaire.

Passer à la placette suivante.

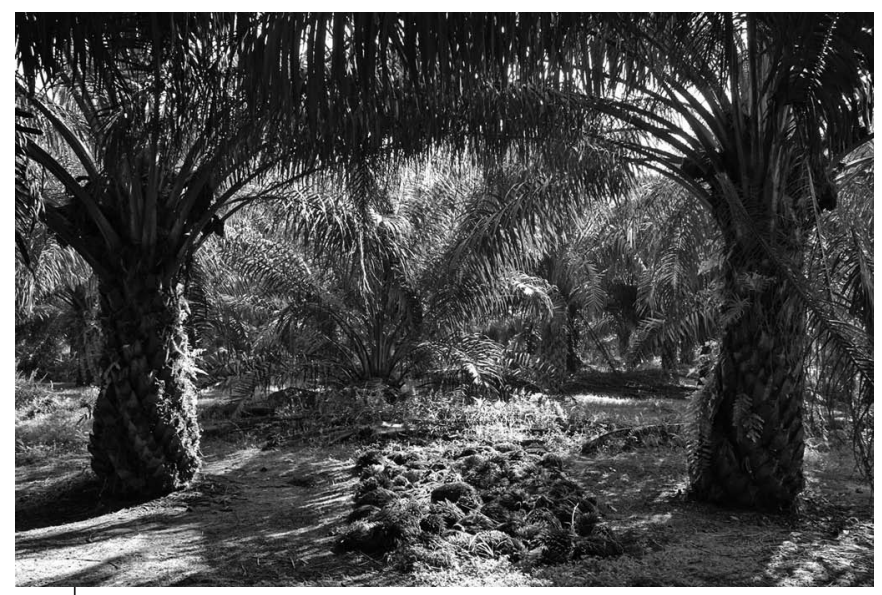

Figure 39.1.

Placette rectangulaire entre deux palmiers. 


\section{Fiche 40. Surveillance phytosanitaire}

La surveillance phytosanitaire est indispensable pour déceler à temps les différents dégâts de ravageurs, les maladies ou les désordres qui peuvent affecter les palmiers. Il est donc nécessaire de :

- contrôler tous les palmiers plantés une fois par mois;

- noter ou faire noter les observations dans un cahier.

Toute modification importante de l'état de santé des arbres : feuilles qui se dessèchent rapidement, feuilles cassées, folioles dévorées ou trouées, nombreux régimes qui avortent ou qui pourrissent, changement de couleur des folioles, etc. doit être notée.

En cas d'évolution importante et préjudiciable au potentiel des arbres, se reporter au chapitre 7 «Défenses des cultures» et, si nécessaire, faire appel au conseiller agricole le plus proche. 


\section{Défense des cultures}

Avec l'aide du conseiller de sa coopérative ou de celui de la plantation commerciale la plus proche, le planteur devra essayer de développer des stratégies de lutte contre les maladies et les ravageurs vers une lutte biologique ou si cela ne peut se faire vers une lutte chimique raisonnée. Les applications de produits phytosanitaires se feront sur la base de seuils techniques et économiques et non par applications prophylactiques.

Le planteur, avec l'appui du conseiller, veillera à délimiter précisément les zones à traiter et les périodes pour le faire. Il I utilisera les matières actives les moins nocives possibles pour
lui-même et l'environnement et prendra soin de suivre toutes les consignes de sécurité.

Si nécessaire, le planteur s'orientera à la création de sa palmeraie ou à sa replantation vers un matériel végétal tolérant ou résistant.

Dans cet ouvrage, ne sont repris que les maladies les plus importantes, les ravageurs principaux et les problèmes nutritionnels les plus fréquemment rencontrés en Afrique de l'Ouest et en Afrique centrale. Dans chacune des fiches, les problèmes sont présentés par stade de culture, puis, les principaux symptômes ou dégâts sont décrits et enfin l'agent responsable de façon à ce que le planteur puisse déterminer la source du problème. Les méthodes de contrôle possibles sont présentées. 
Maladies identifiées - Fiche 41.

\begin{tabular}{ll}
\hline Stade cultural & Maladie \\
\hline Pépinière & Blast \\
\hline Pépinière et jeunes cultures & Pourriture sèche du cœur \\
\cline { 2 - 2 } & Cercosporiose \\
\hline Plantations adultes & Fusariose \\
\hline
\end{tabular}

Ravageurs répertoriés dans ce guide - Fiche 42.

\begin{tabular}{ll}
\hline Stade cultural & Ravageur \\
\hline Pépinière & Temnoscoites \\
\hline \multirow{2}{*}{ Jeunes cultures } & Rats et agoutis \\
\cline { 2 - 2 } & Criquets puants \\
\cline { 2 - 2 } Jeunes cultures et plantation en rapport & Rhynchophorus \\
\hline & Coelaenomenodera \\
\cline { 2 - 2 } Plantation en rapport & Hespérides \\
\cline { 2 - 2 } & Limacodides \\
\hline
\end{tabular}

Autres problèmes sanitaires identifiés - Fiche 43.

\begin{tabular}{ll}
\hline Stade sensible & Problème sanitaire \\
\hline Pépinière, jeunes cultures & Déficience azotée \\
\hline \multirow{3}{*}{ Plantations adultes } & Déficience potassique \\
\cline { 2 - 2 } & Déficience magnésienne \\
\cline { 2 - 2 } & Impact de la foudre \\
\hline
\end{tabular}




\section{Fiche 41. Principales maladies rencontrées en Afrique de l'Ouest et en Afrique équatoriale}

Les figures sont placées dans le cahier couleur.

ID Stade sensible : pépinière

Blast

\section{Symptômes}

Pourriture de la flèche et des racines puis dessèchement des feuilles; section du bulbe orangée; mortalité importante. Apparaît de septembre à mars en Afrique de l'Ouest (figures 15.1 et 41.1).

\section{Agent causal}

Non déterminé.

\section{Agent vecteur}

Un insecte piqueur : Recilia mica, Homopterae, Jassidae.

\section{Méthode de contrôle préventive}

Conduire la pépinière sous ombrière de septembre à mars (voir Fiche 9).

Les graminées étant les refuges du vecteur, intensifier le désherbage de la pépinière et l'élimination des graminées dans ses abords (voir Fiche 12).

\section{Méthode de contrôle curative}

Arracher et brûler les plants malades.

En cas de rémission, ne pas hésiter à détruire les plants concernés.

Le blast apparaît rarement en prépépinière ou en première année de plantation. Dans ce dernier cas, cela peut être dû à une défaillance de la sélection en fin de pépinière.

\section{ID Stade sensible : pépinière et période immature}

\section{Pourriture sèche du cœur}

\section{Symptômes}

Arrêt de la croissance du plant et de l'émission foliaire; petites taches jaunâtres sur le limbe à l'extrémité de la feuille 1 de part et d'autre de la nervure centrale; taches d'aspect huileux ou aspect liégeux sur la coupe du bulbe; parfois coloration violacée du plateau racinaire. Apparaît à partir de décembre-janvier (figures 15.2 et 41.2). 


\section{Agent causal}

Non déterminé.

\section{Agents vecteurs}

Des insectes piqueurs : Sogatella cubana et S. kolophon; Homopterae, Delphacidae.

\section{Méthodes préventive et curative de contrôle}

Se reporter à Blast ci-dessus.

La pourriture sèche du cœur peut apparaître en première année de plantation. Dans ce cas, il faut extirper le plant malade et le remplacer.

\section{Cercosporiose}

\section{Symptômes}

Mouchetures irrégulières de couleur orangée puis brune sur les folioles des feuilles les plus âgées. Se développe progressivement sur les feuilles plus jeunes. En fin d'évolution, dessèchement du limbe en commençant par les extrémités des folioles. Apparaît souvent en relation avec des conditions de stress hydrique notamment. Affecte tous les stades de culture de la pépinière à la plantation en rapport. (cahier couleur figure 15.4)

\section{Agent causal}

Champignon foliaire Cercospora elaeidis.

\section{Méthode de contrôle}

Vérifier la qualité de l'arrosage et corriger celui-ci si nécessaire en particulier pendant les périodes sèches.

Dès que plus de $10 \%$ des plants sont atteints, mettre en place le traitement antifongique décrit dans la Fiche 12.

En pépinière, il n'est pas recommandé d'ôter les feuilles malades car cela accentue l'effet dépressif.

La cercosporiose a un effet dépressif sur la croissance des plants en particulier en pépinière. Des poussées de cercosporiose peuvent être observées en saison sèche pendant la période immature. En conditions normales, elle n'a pas d'incidence économique à l'âge adulte. Certains types de matériel végétal sont plus sensibles que d'autres. 


\section{Stade sensible : plantation adulte et jeune culture}

\section{Fusariose}

\section{Symptômes}

Aspect typique : quelques flèches non ouvertes; jeunes feuilles plus courtes; jaunissement puis dessèchement des feuilles de la couronne moyenne; cassure du rachis au premier tiers. Mort fréquente (figure 41.3).

Aspect chronique : nombreuses flèches non ouvertes; réduction forte de la longueur des feuilles; stipe en forme de pointe de crayon; présence fréquente de crevasses. Production de régimes très faible à nulle (figure 41.4).

En replantation jeune : feuilles moyennes jaunissantes et raccourcissement de la longueur des jeunes feuilles.

\section{Agent causal}

Un champignon du sol Fusarium oxysporum sp. elaeidis.

\section{Lutte intégrée}

La maladie se propage de proche en proche par contact des racines du palmier avec celles d'un palmier atteint de la maladie ou de son bulbe racinaire. La lutte doit donc tenir compte de ce facteur très important : - éradication périodique des arbres malades; creusement d'une fosse de 1,5 x 1,5 x 1 m à l'emplacement du bulbe racinaire; exporter le bulbe malade hors de la parcelle, sinon, l'exposer au soleil;

- en replantation, mettre en place un matériel végétal certifié tolérant à la maladie; replanter le plus loin possible des anciennes souches.

La fusariose est une maladie grave et souvent mortelle en plantation. Elle apparaît vers 8-10 ans. Présence de fibres brunes dans le cour ou le stipe. En replantation, c'est une maladie du jeune âge. Si la replantation est effectuée avec un matériel végétal certifié tolérant, de nombreuses rémissions sont observées.

En Afrique équatoriale, une autre maladie provoquée par un champignon du sol peut apparaître sur des arbres déjà affectés par la fusariose, le Ganoderma. Les symptômes sont proches avec en plus l'émission à la base du stipe de carpophores du champignon (figure 41.5). La méthode de contrôle est identique à celle de la fusariose. 


\section{Fiche 42. Principaux ravageurs rencontrés en Afrique de l'Ouest et en Afrique équatoriale}

\section{Les figures sont placées dans le cahier couleur.}

\section{II) Stade sensible : pépinière}

\section{Larve du petit coléoptère Temnoschoita quadripustulata}

\section{Symptômes}

Les bases pétiolaires et parfois le bulbe lui-même abritent la larve, un petit «ver» blanc. La feuille attaquée ou le plant lui-même finit par se dessécher. Les dégâts apparaissent le plus souvent en fin de pépinière ou, plus rarement, en tout début de plantation (cahier couleur, figure 15.6).

\section{Agent causal}

Temnoschoita quadripustulata (Curculionidae, Rhynchophorinae).

\section{Méthode de contrôle préventive}

Nettoyer les abords de la pépinière. Les dégâts font toujours suite à des pontes de l'insecte sur des blessures, donc, éviter de couper les feuilles à moins de $15 \mathrm{~cm}$ du bulbe lors des habillages en pépinière et avant la plantation.

Méthode de contrôle curative

Détruire les larves manuellement si l'attaque n'affecte qu'une ou deux feuilles. Détruire le plant si un plus grand nombre de feuilles sont attaquées. En cas d'attaques régulières, en plus de la procédure ci-dessus, contacter le conseiller pour effectuer un traitement de la pépinière avec un insecticide systémique (voir Fiche 15).

Ladulte de Temnoschoita quadripustulata est un petit coléoptère reconnaissable aux 4 taches noires sur ses élytres. Il est très commun dans les plantations de palmier adulte et sans incidence économique.

\section{Stade sensible : jeune culture}

\section{«Agoutis 》}

\section{Symptômes}

Les bases pétiolaires sont rongées, parfois coupées. Lorsque les plants sont très jeunes, le bulbe lui-même peut être sectionné (figure 42.1). Ces blessures servent souvent de porte d'entrée à d'autres ravageurs comme les temnoschoites ou les rhyncophores. 


\section{Agents causaux}

«Agoutis» ou Thryonomys swinderianus.

Petits rats dont Dasymys sp. ou Lemniscomis spp.

\section{Méthode de contrôle préventive}

Élargir les ronds à $3 \mathrm{~m}$ de diamètre; mettre en place les grillages en spirales (voir Fiche 26).

\section{Méthode de contrôle curative}

Chasser les rongeurs avec des chiens. En dernier recours, poser des appâts empoisonnés à base de bromodiolone, de chlorophacinone ou de coumafène.

\section{Attention}

Les grands et petits ruminants sont extrêmement sensibles au poison des appâts. Il ne faut surtout pas les laisser pâturer dans les parcelles où ceux-ci ont été posés.

\section{Criquet Zonocerus variegatus}

\section{Symptômes}

Face supérieure des folioles décapée par plaques puis, un peu plus tard, toute l'épaisseur des folioles est découpée (figures 42.2 et 42.3). Présence d'insectes de couleur jaune et noir sur les feuilles.

\section{Agent causal}

Tous les stades larvaires et les adultes du criquet Zonocerus variegatus.

\section{Méthode de lutte préventive}

Détruire les touffes de Chromolaena odorata présentes dans la plantation.

\section{Méthode de lutte curative}

Pulvériser sur les arbres et la végétation environnante un des produits suivants :

- Deltaméthrine

- Cyperméthrine

- Alpha-cyperméthrine

- Lambda-cyhalothrine

Les larves des premiers stades de Zonocerus variegatus vivent en colonies. Cet insecte se développe abondamment sur les touffes de Chromolaena odorata qui servent ainsi de réservoir. L'insecte émet une odeur repoussante lorsqu'il est attaqué d'où son surnom de «criquet puant». Il peut apparaître sur les plants de fin de pépinière si les abords sont envahis par Chromolaena odorata. 


\section{Adultes du scarabée Oryctes monoceros}

\section{Symptômes}

Galeries de 1,5 à $2 \mathrm{~cm}$ de diamètre dans les pétioles vers la zone d'insertion de la feuille au bulbe ou à la base des flèches; la galerie peut parfois atteindre le bulbe et mettre en danger la survie du jeune arbre. Sur des arbres de 3 ans et plus, les dégâts sur les flèches sont très reconnaissables après leur ouverture par les découpes caractéristiques des folioles en arêtes de poisson (figure 42.4).

\section{Agent causal}

Adultes du scarabée Oryctes monoceros (Coléoptère, Scarabeidae) (figure 42.5).

\section{Méthode de lutte préventive}

Destruction des stipes des palmiers morts; élimination ou nettoyage des gîtes larvaires avant une replantation (bois pourri, tas de compost, stipes en décomposition); collecte et destruction des larves; visiter la plantation chaque semaine, extirper et détruire les adultes présents sur les arbres.

\section{Méthode de lutte chimique raisonnée}

Effectuer des recensements mensuels; traiter avec de l'alphacyperméthrine et du lambda-cyhalothrine en alternance tous les 10 jours sur les flèches tant que le niveau d'attaque atteint ou dépasse $4 \%$ par interligne. Maintenir le traitement 2 mois après la fin de l'alerte.

Les attaques se produisent aux premières heures de la nuit ou à l'aube.

\section{ID Stade sensible : jeune culture et culture en production}

\section{Larves du gros charançon Rhyncophorus phoenicis}

\section{Symptômes}

Présence de gros «vers blancs» dans les tissus vivants du bulbe (arbres jeunes) ou du stipe (arbres adultes); bruits de crissement ou de craquements audibles provenant de l'intérieur de l'arbre; dessèchement progressif et mort de l'arbre.

\section{Agent causal}

Larves du gros charançon Rhynchophorus phoenicis (Coléoptère, Curculionidae) (figure 42.6).

\section{Méthode de lutte préventive}

Vérifier la présence et l'origine de blessures éventuelles. Éviter toute cause de blessure profonde aux arbres notamment lors de l'élagage ou 
de la castration si la présence de l'insecte est avérée. Si les blessures sont provoquées par un autre ravageur, appliquer une méthode de lutte appropriée contre celui-ci.

\section{Méthode de lutte curative}

Abattre les arbres atteints et détruire les larves. Contacter le conseiller pour mettre en place des piégeages.

Ladulte de Rhyncophorus sp. n'attaque pas les palmiers. La femelle pond toujours dans une blessure occasionnée soit par un autre ravageur soit par des pratiques culturales inadaptées.

\section{II) Stade sensible : Plantation en exploitation}

\section{Larves de Coelaenomenodera lameensis ou de C. minuta}

\section{Symptômes}

Épiderme supérieur des folioles boursouflé et desséché; présence de petites galeries dans l'épaisseur de la foliole (figure 42.7); dessèchement progressif des feuilles en cas d'attaques prononcées.

\section{Agent causal}

Larves de Coelaenomenodera lameensis ou de C. minuta (Coleoptères, Chrysomelidae).

\section{Méthode de lutte}

En raison du matériel nécessaire, et étant donné la capacité de l'insecte à développer de fortes pullulations et à passer d'une plantation à l'autre, la lutte intégrée contre ce ravageur ne peut se faire que de façon concertée avec les autres planteurs. Elle doit se mettre en place avec l'appui de la coopérative ou de la plantation commerciale la plus proche.

Le cycle larvaire et la nymphose de $C$. lameensis ou de $C$. minuta se font dans la galerie. Les adultes de couleur rougeâtre se rencontrent sur la face inférieure des folioles où ils creusent de petits sillons pour se nourrir (Figure 42.8).

\section{Chenille de Pteroteinon laufella}

\section{Symptômes}

Défoliation plus ou moins complète des folioles pouvant atteindre près de $100 \%$ en ne laissant que la nervure centrale. Le bord des folioles est replié en cornet par le ravageur. Les palmes défoliées ressemblent à une arête de poisson (figure 42.9). 


\section{Agent causal}

Larve de Pteroteinon laufella (Lépidoptère, Hesperidoidea).

\section{Méthode de lutte}

En raison de la capacité de l'adulte à passer d'une plantation à l'autre et du matériel nécessaire, la lutte intégrée contre ce ravageur ne peut se faire que de façon concertée avec les autres planteurs. Elle doit se mettre en place avec l'appui de la coopérative ou de la plantation commerciale la plus proche.

La chenille de dernier stade mesure $5 \mathrm{~cm}$ de long (Figure 42.10). Elle fabrique le cornet qui l'abrite en collant les bords du limbe de la foliole par un fil de soie.

\section{Chenille de Latoia spp.}

\section{Symptômes}

Défoliation plus ou moins complète des folioles pouvant atteindre près de $100 \%$ en ne laissant que la nervure centrale. Les palmes défoliées ressemblent à une arête de poisson. Il n'y a pas de cornets de folioles.

\section{Agent causal}

Chenille de Latoia spp. (Lépidoptère, Zygaenoidea) (figure 42.11).

\section{Méthode de lutte}

En raison de la capacité de l'adulte à passer d'une plantation à l'autre et du matériel nécessaire, la lutte intégrée contre ce ravageur ne peut se faire que de façon concertée avec les autres planteurs. Elle doit se mettre en place avec l'appui de la coopérative ou de la plantation commerciale la plus proche.

La chenille de Latoia spp. est vivement colorée et fortement urticante. 

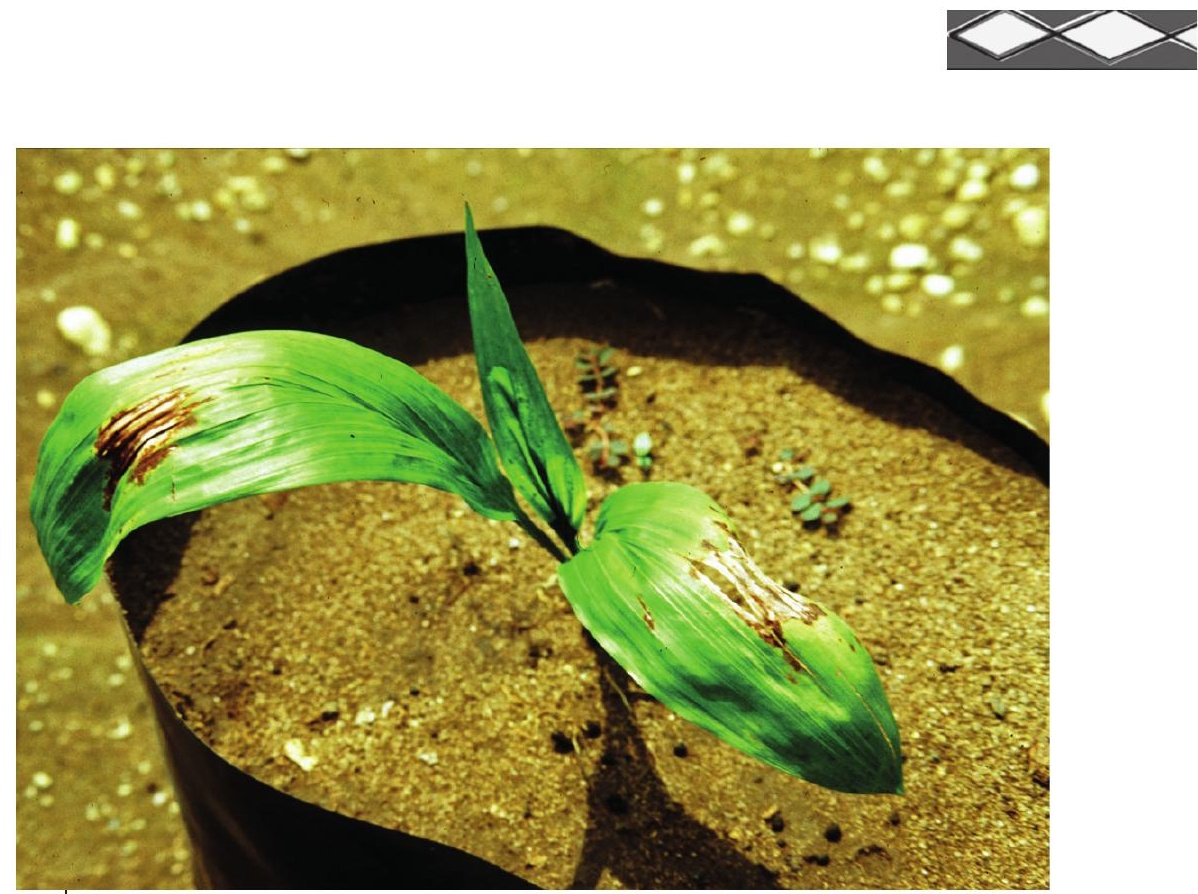

Figure 6.1.

Plantule brûlée par une exposition au soleil trop brutale.

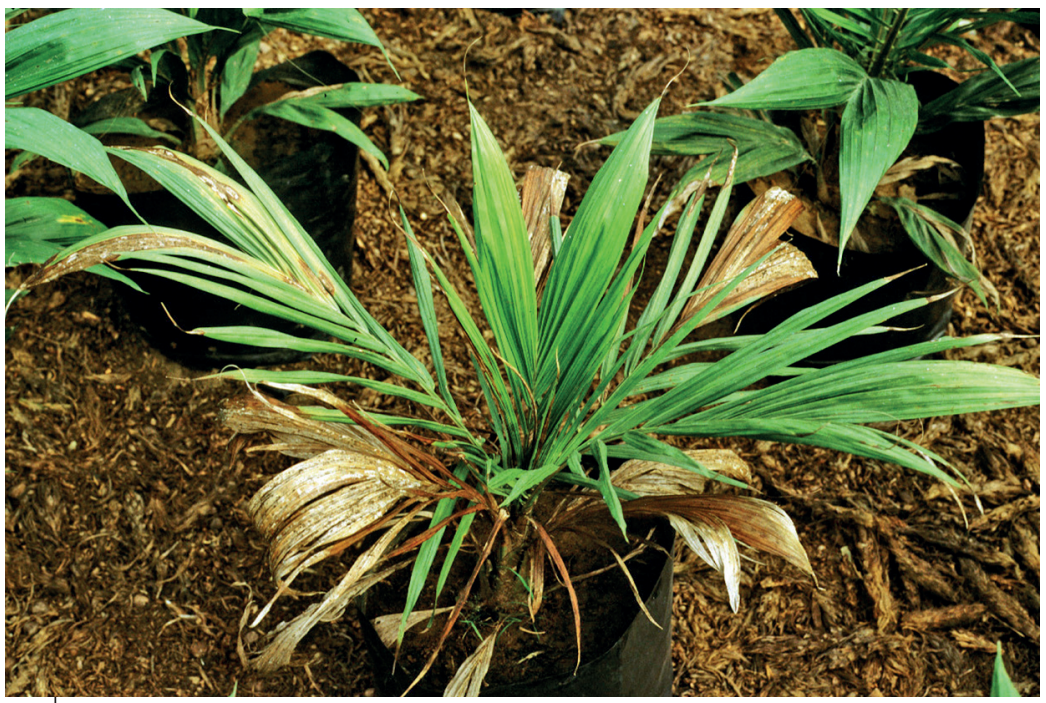

Figure 15.1.

Plant atteint de blast. 
Le palmier à huile en plantation villageoise

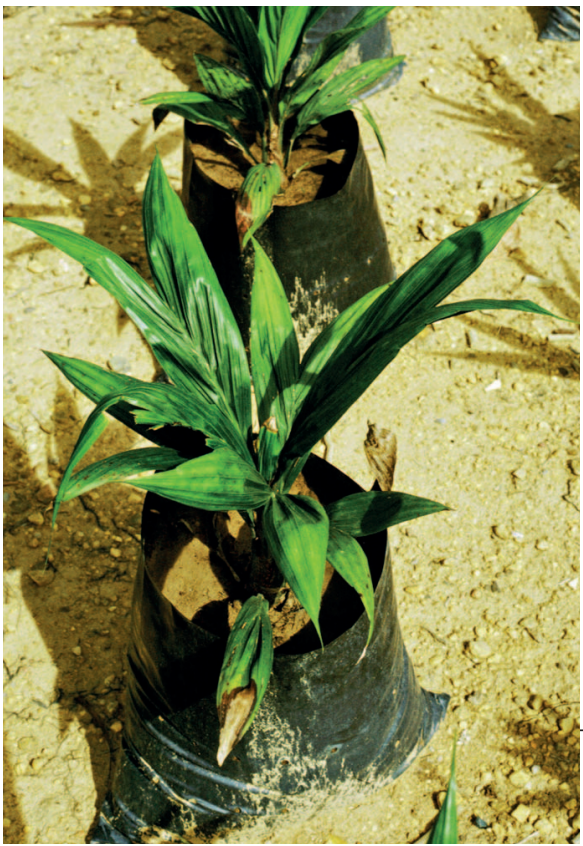

Figure 15.2.

Plant atteint de

pourriture sèche du cœur.

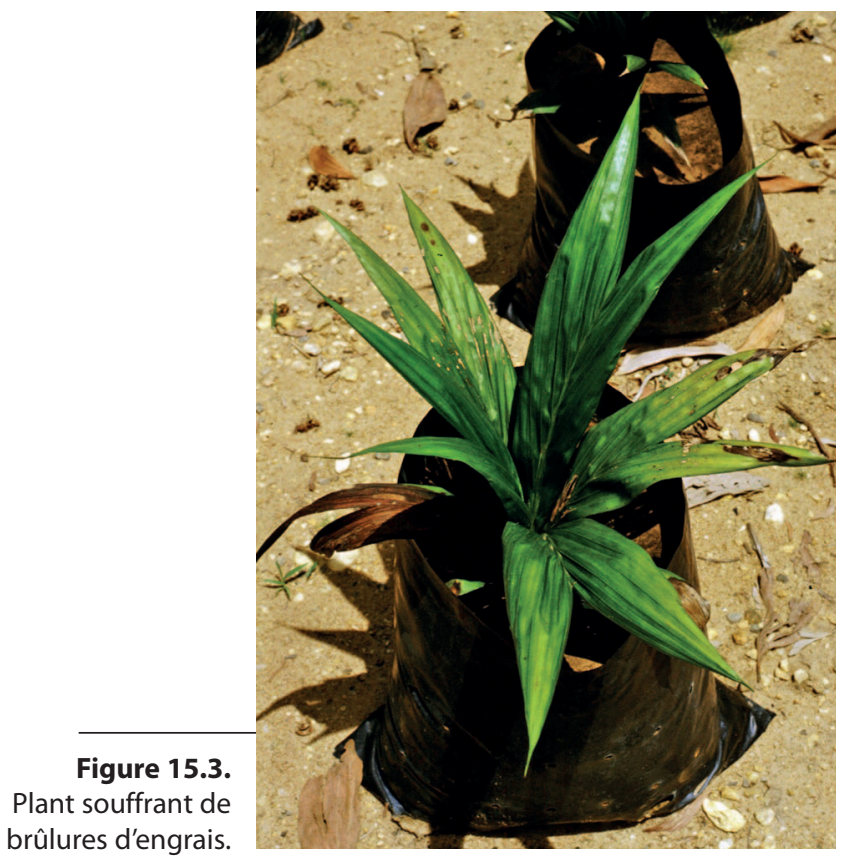



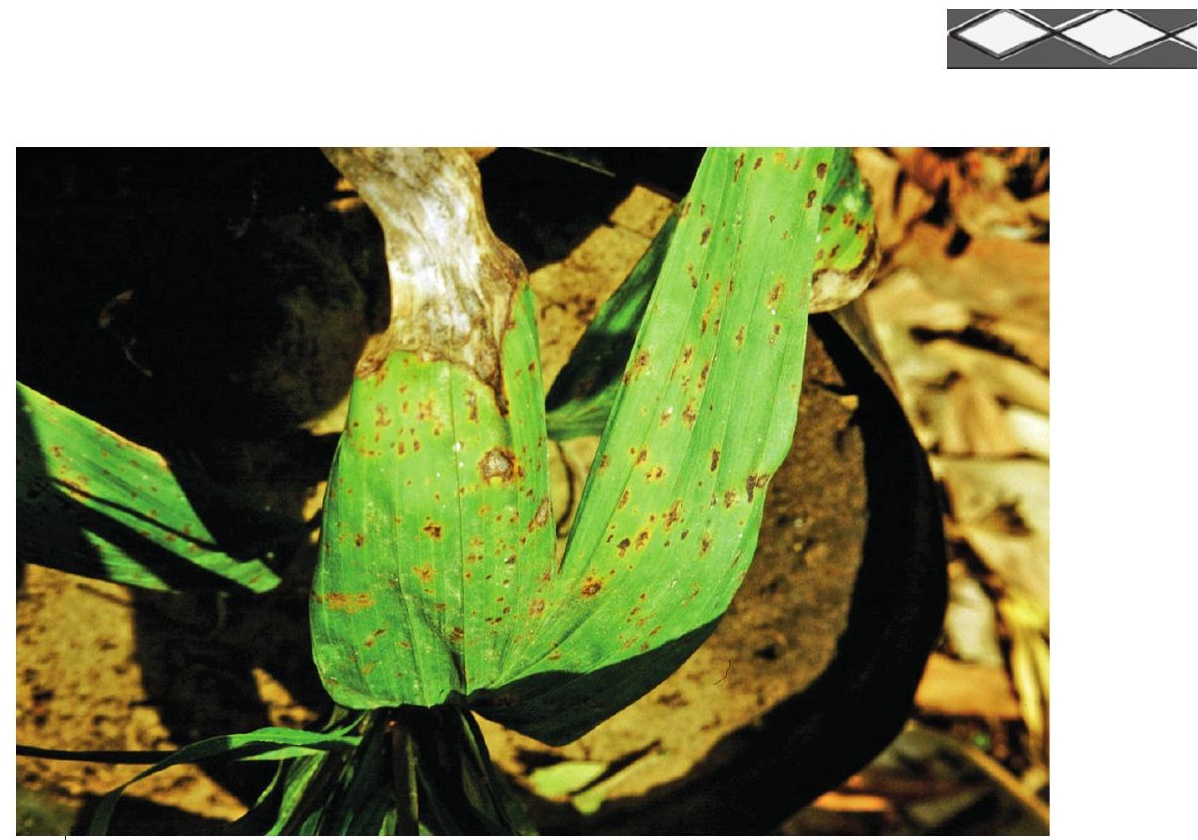

Figure 15.4.

Plant souffrant d'attaque de cercosporiose.

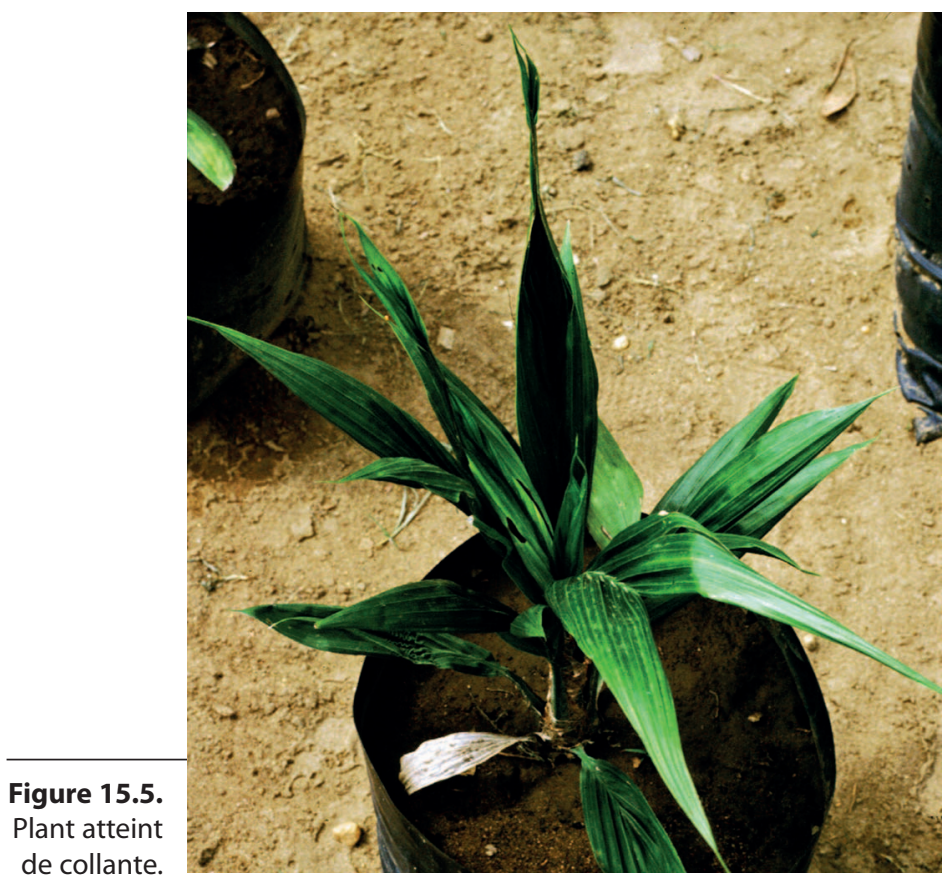




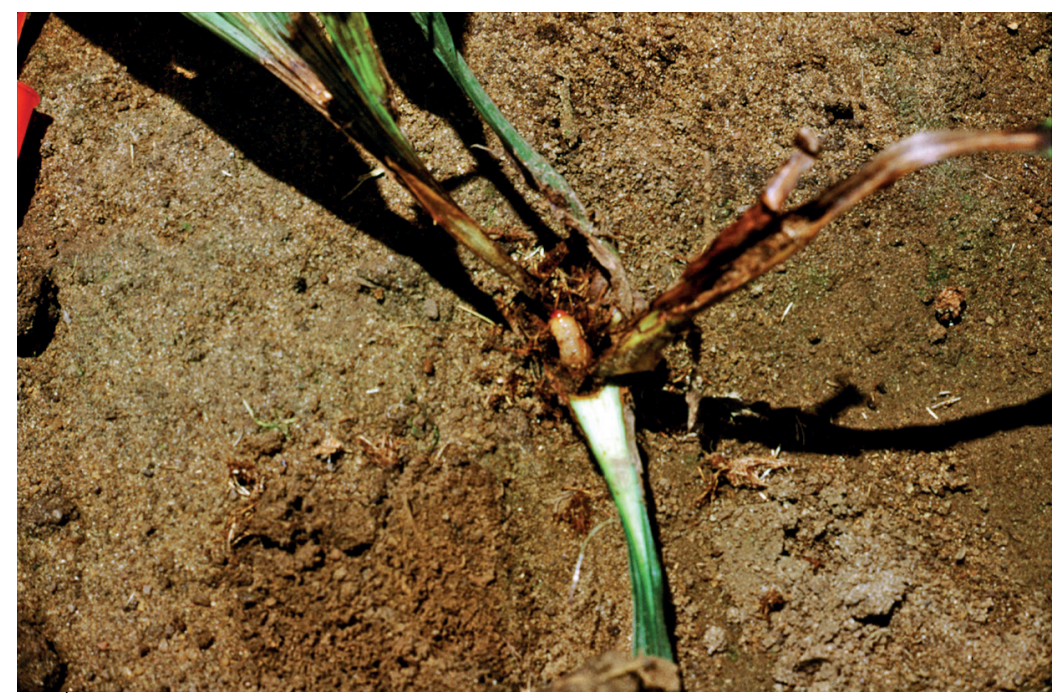

Figure 15.6.

Plant attaqué par des temnoschoites.

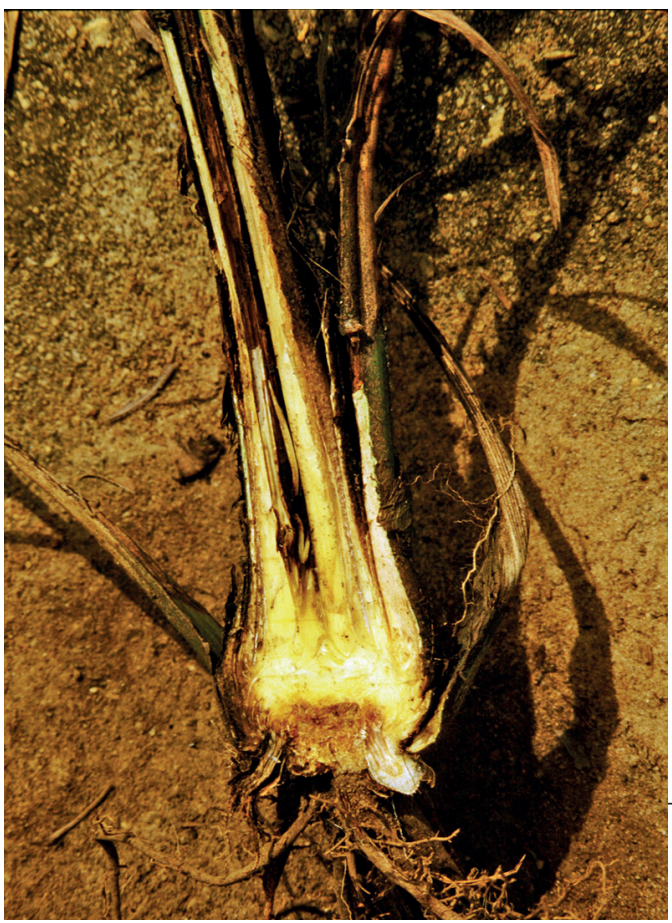

Figure 41.1.

Coupe verticale de plant atteint de blast. 

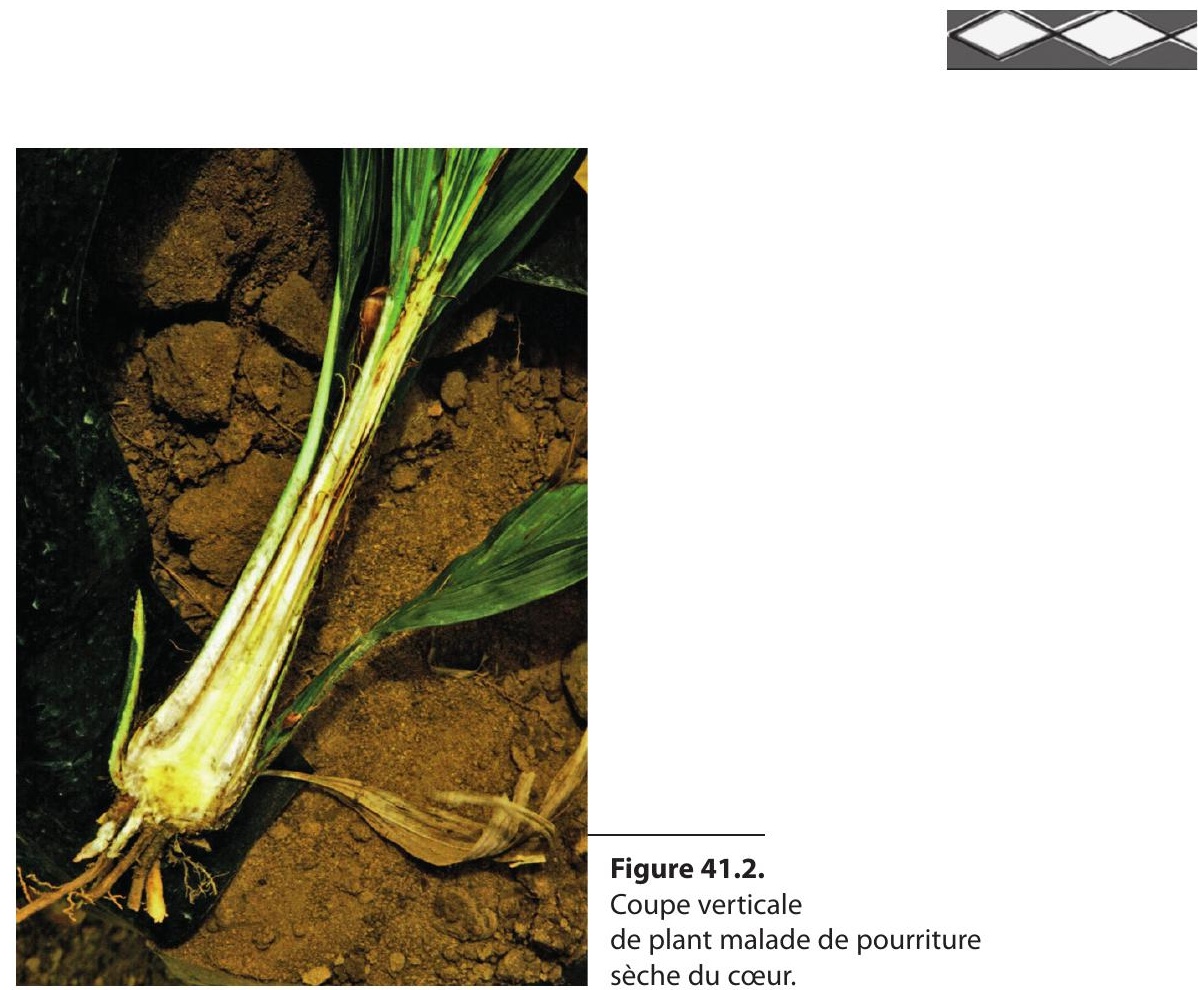

Figure 41.2.

Coupe verticale

de plant malade de pourriture

sèche du cœur.

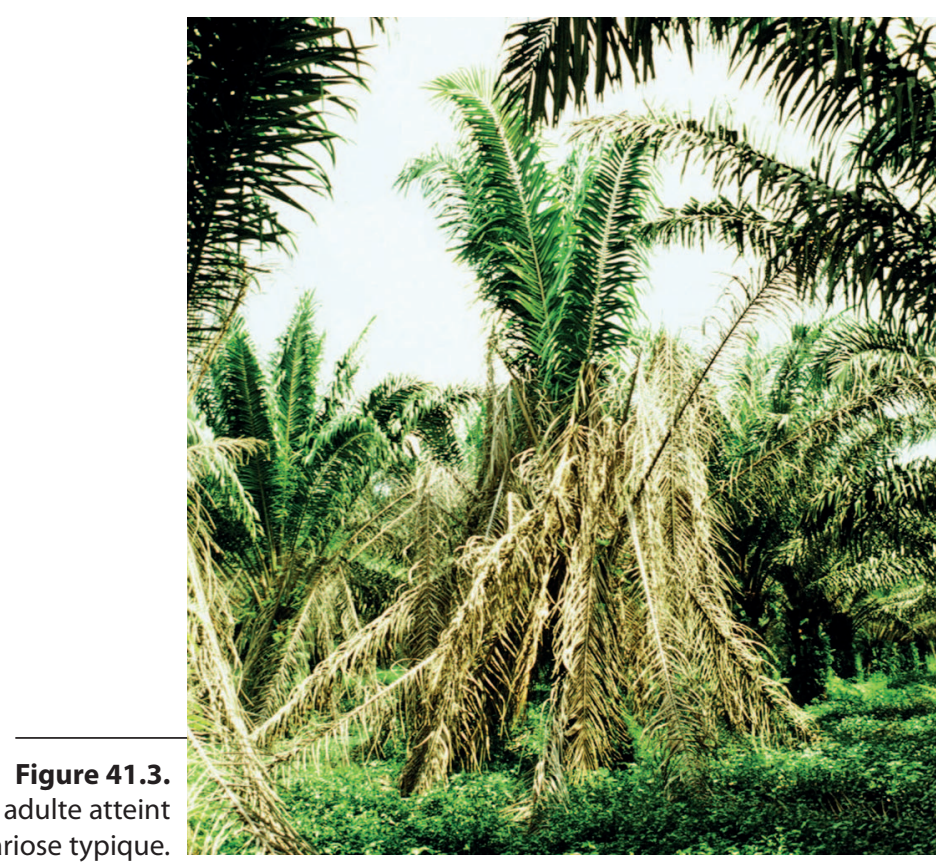

Palmier adulte atteint de fusariose typique. 
Le palmier à huile en plantation villageoise
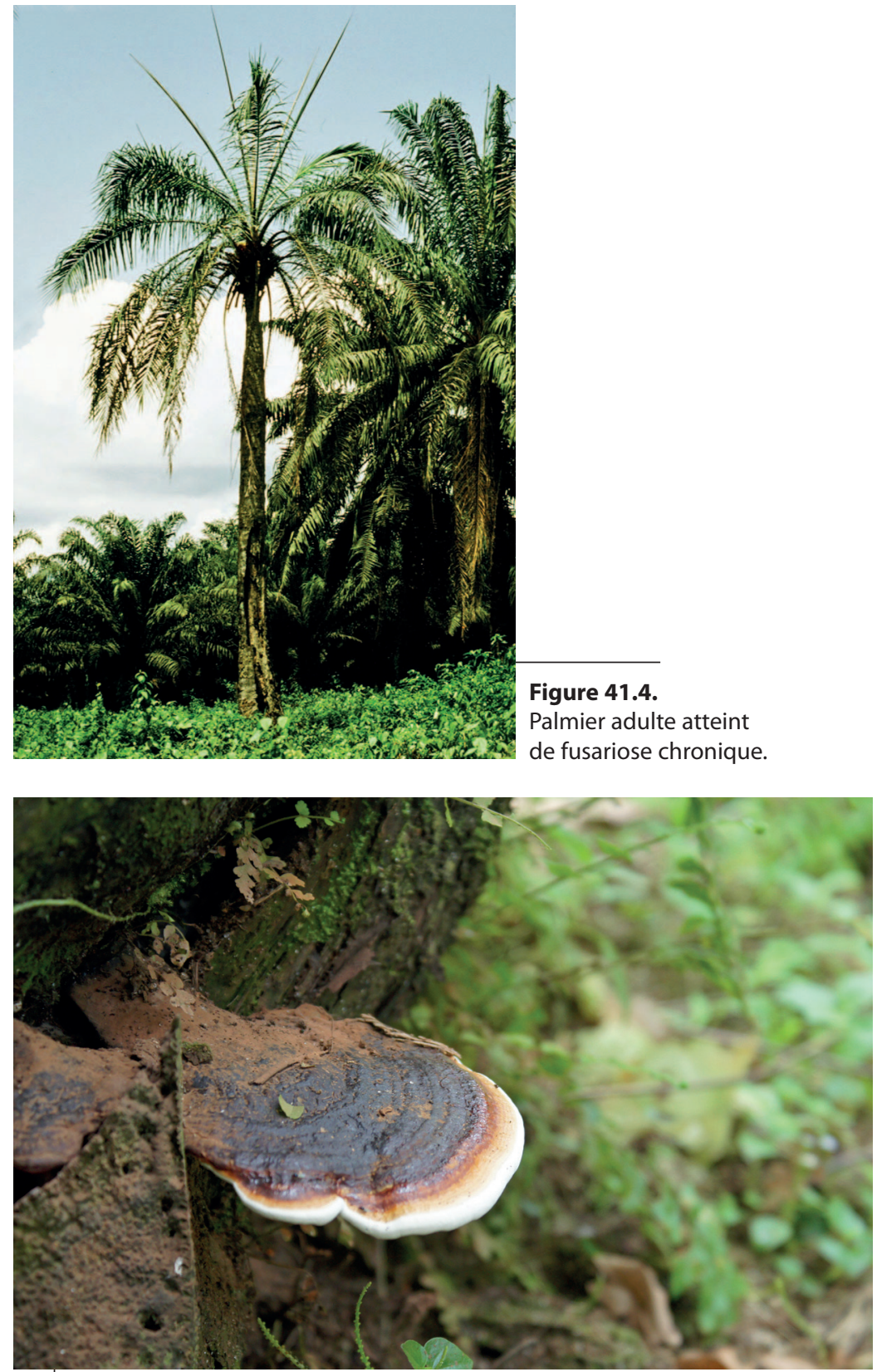

Figure 41.5.

Carpophore de Ganoderma sp. à la base du stipe. 

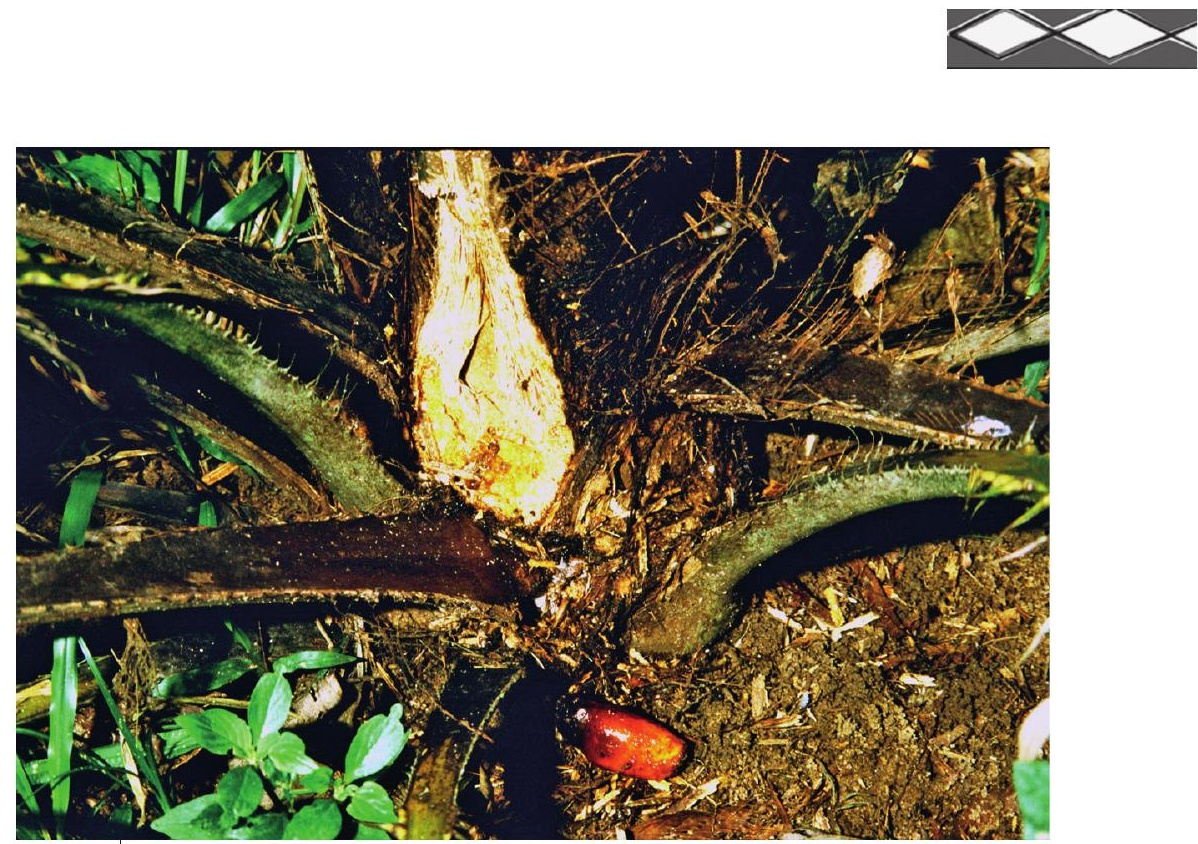

Figure 42.1.

Dégâts provoqués par une attaque de Thryonomys sp.

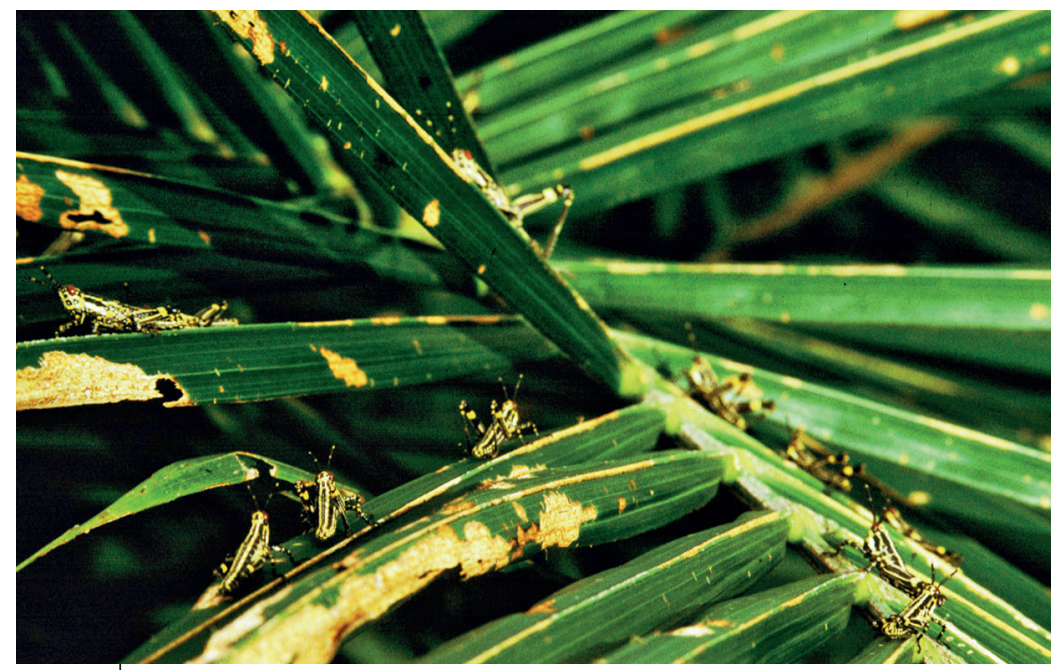

Figure 42.2.

Dégâts provoqués par les premiers stades larvaires

de Zonocerus variegatus. 
Le palmier à huile en plantation villageoise

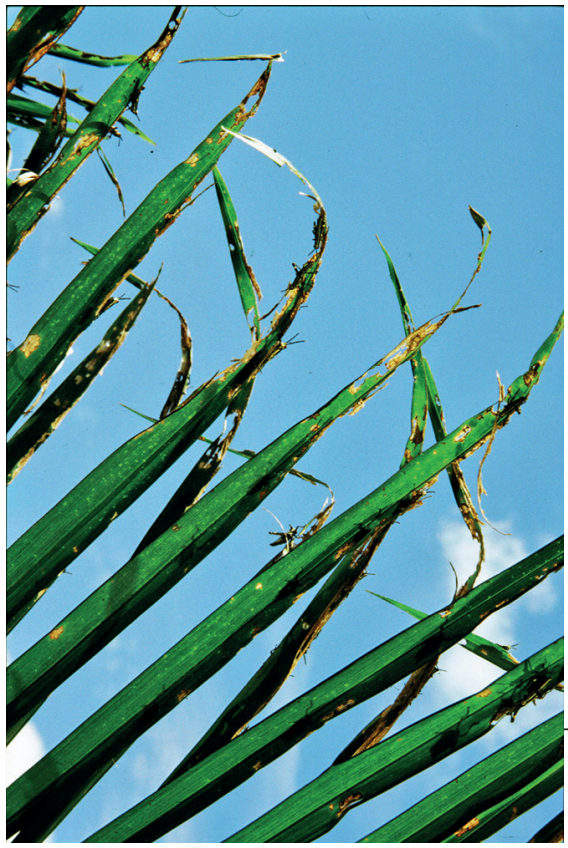

Figure 42.3.

Dégâts provoqués par les adultes de Zonocerus variegatus.

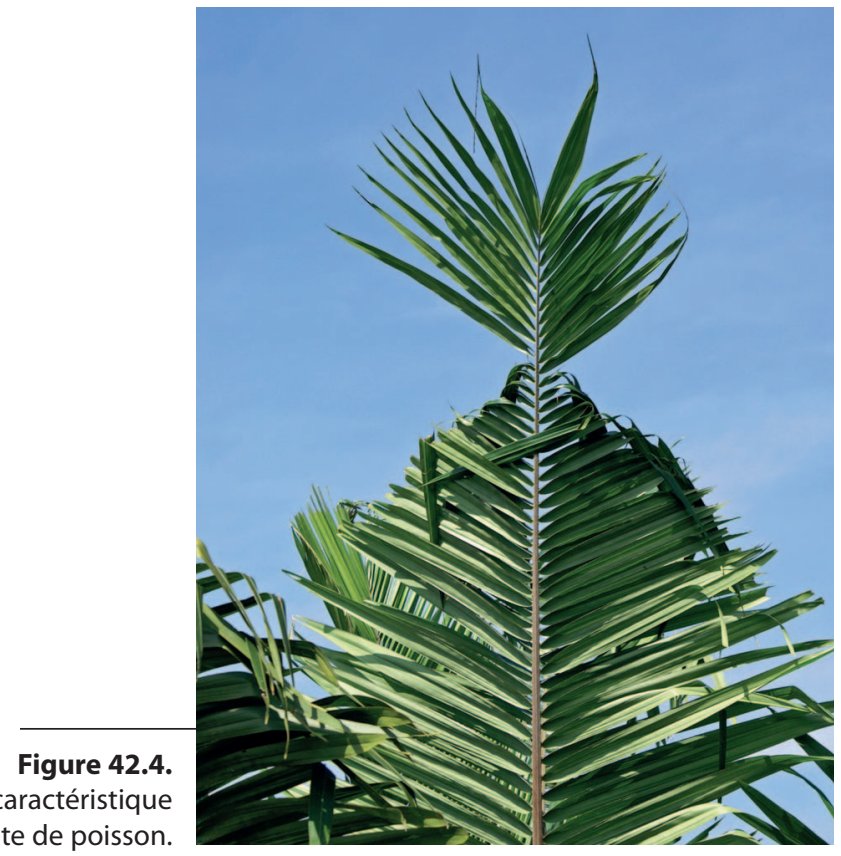

Dégât caractéristique en arête de poisson. 

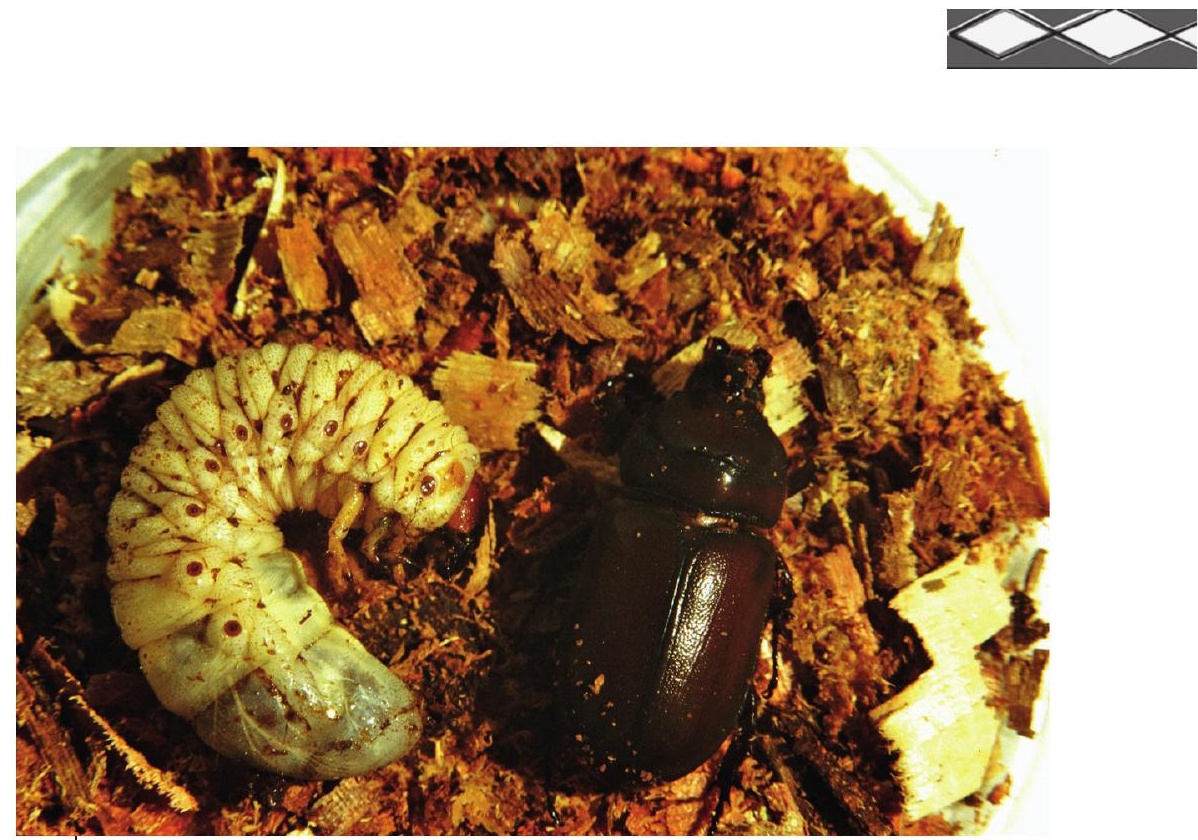

Figure 42.5.

Larve et adulte de Oryctes monoceros.

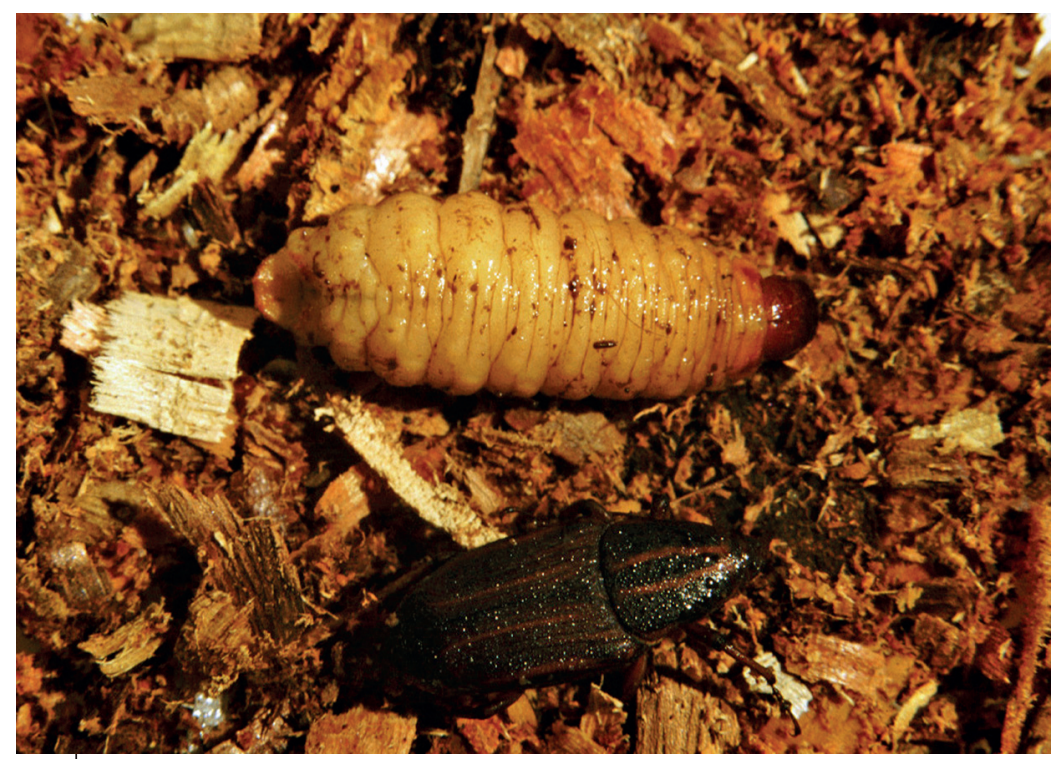

Figure 42.6.

Larve et adulte de Rhynchophorus phoenicis. 


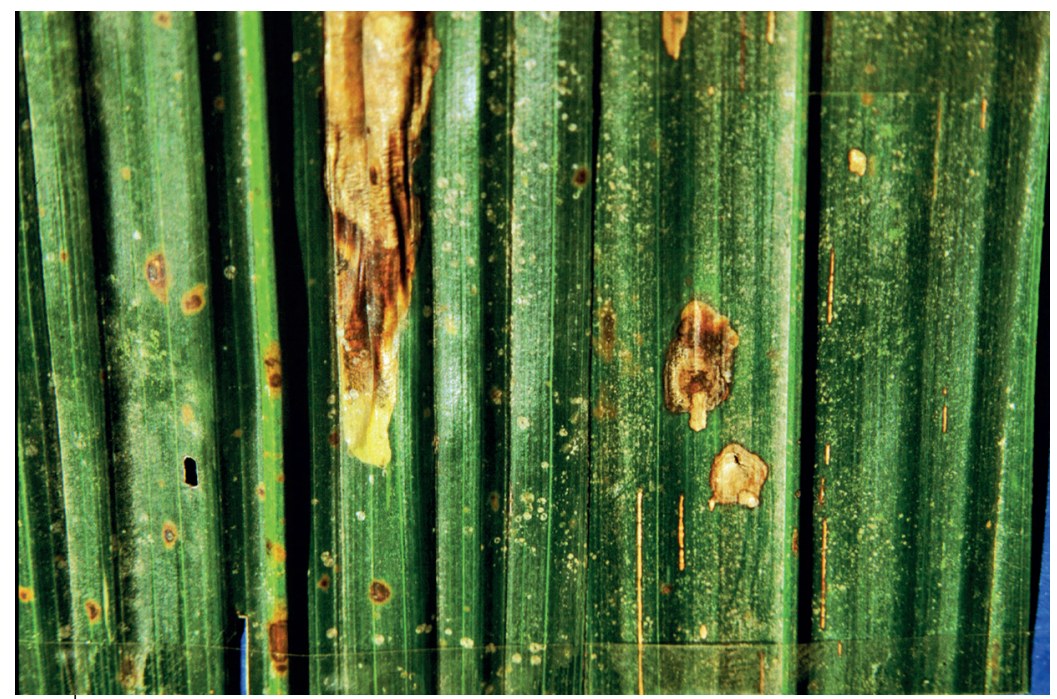

Figure 42.7.

Dégâts caractéristiques provoqués par les larves de $C$. lameensis.

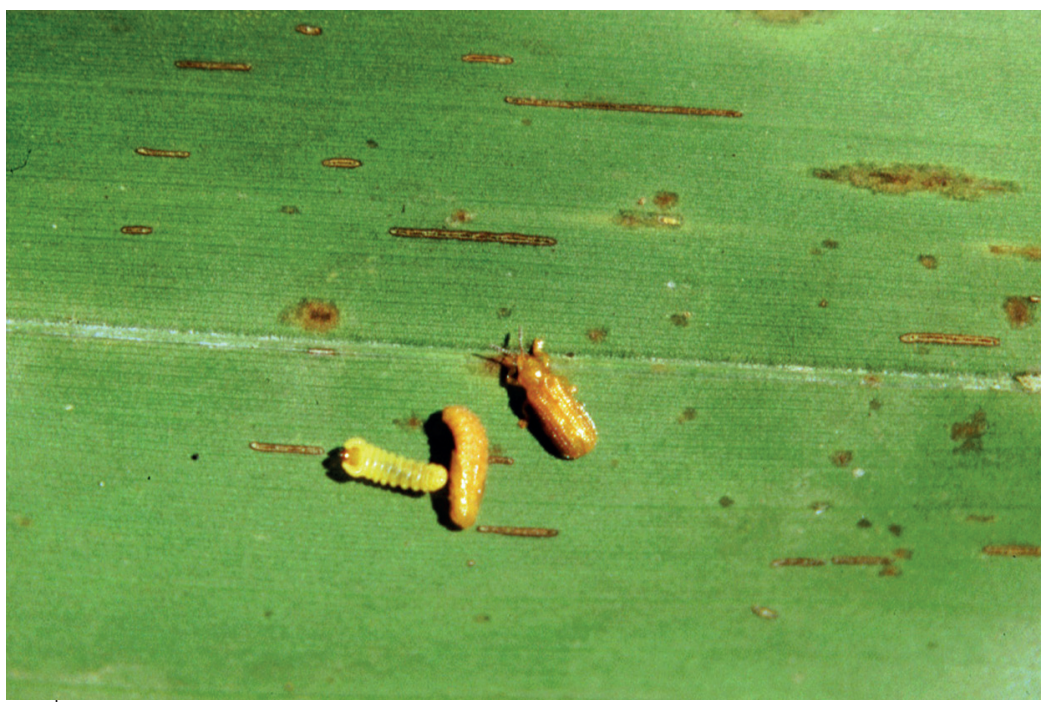

Figure 42.8 .

Adulte et larves de C. lameensis. 


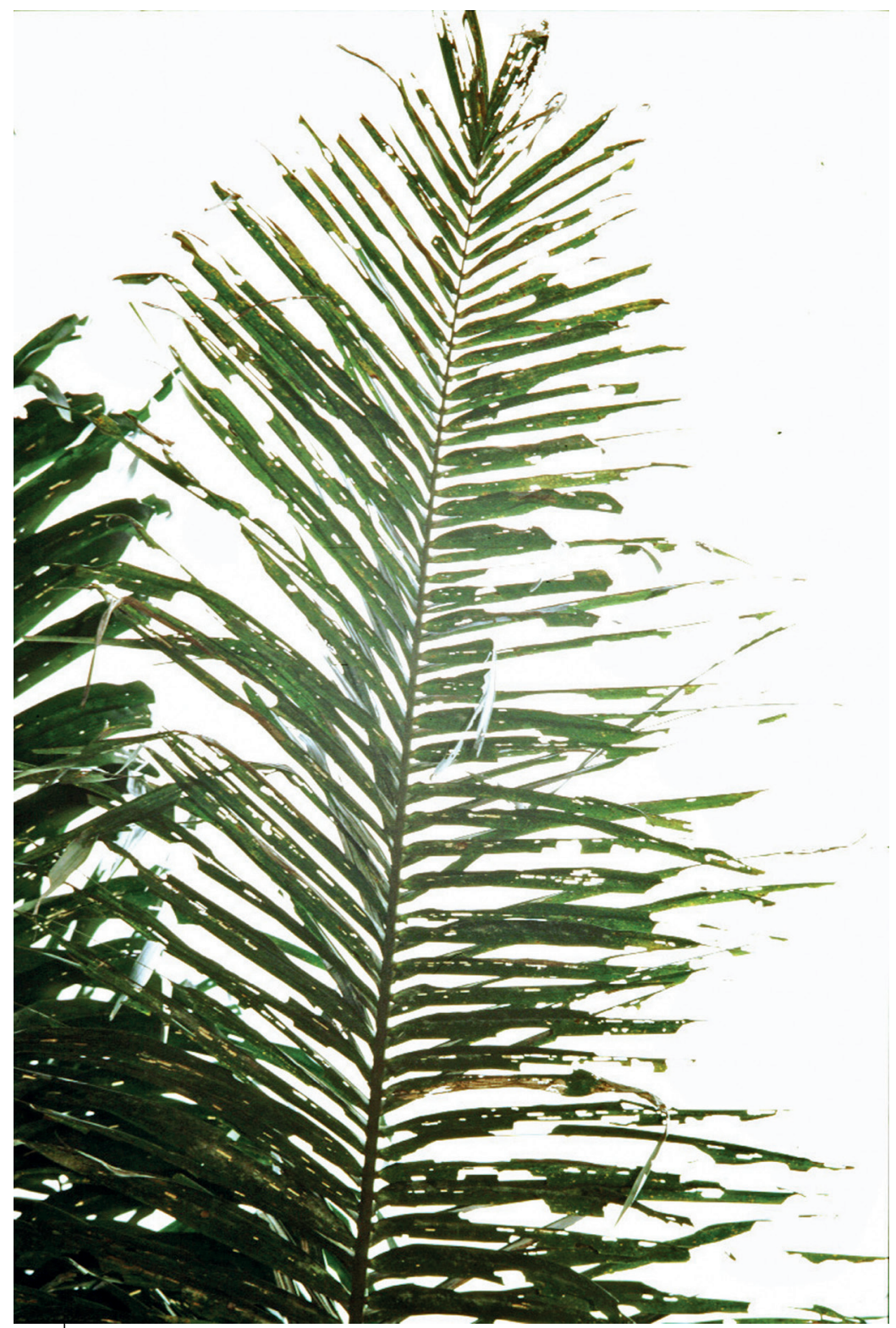

Figure 42.9.

Palme très fortement défoliée par $P$. laufella. 


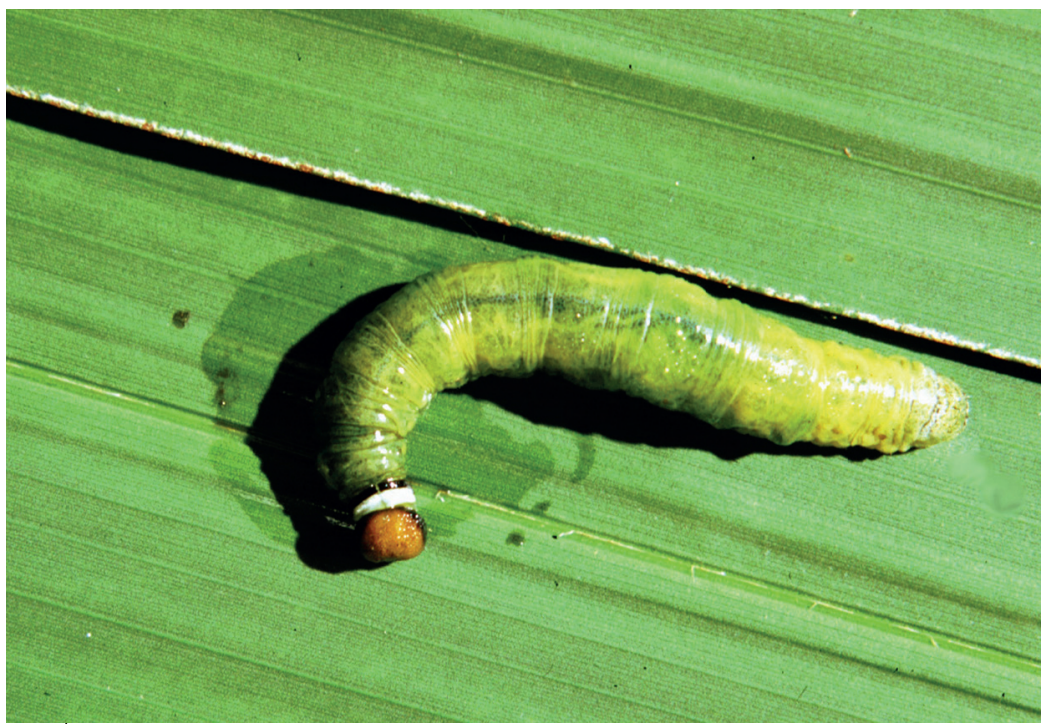

Figure 42.10.

Chenille de dernier stade de P. laufella hors de son cornet.

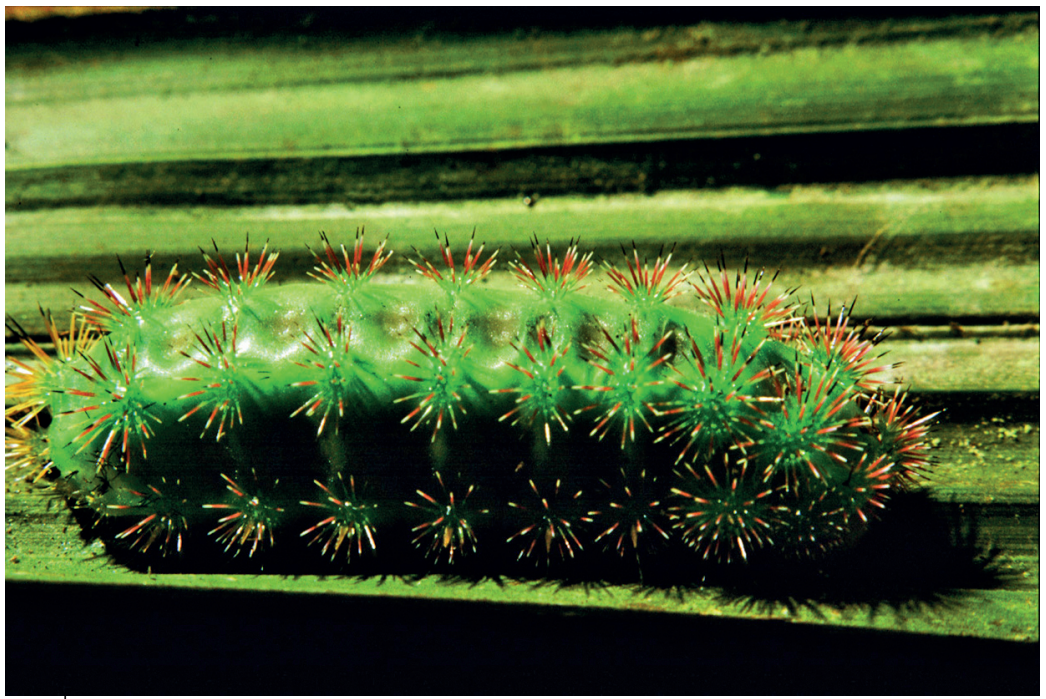

Figure 42.11.

Chenille de Latoia spp. 

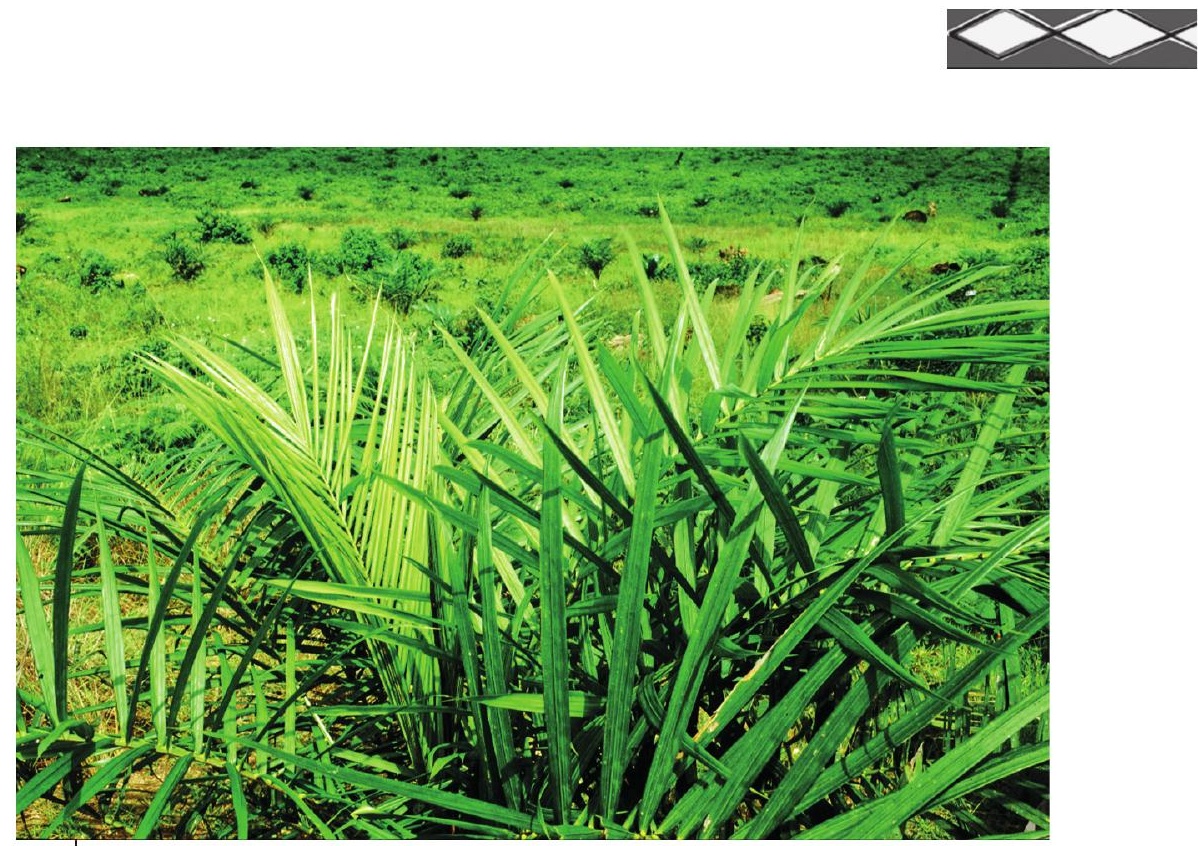

Figure 43.1.

Symptômes caractéristiques de déficience azotée.

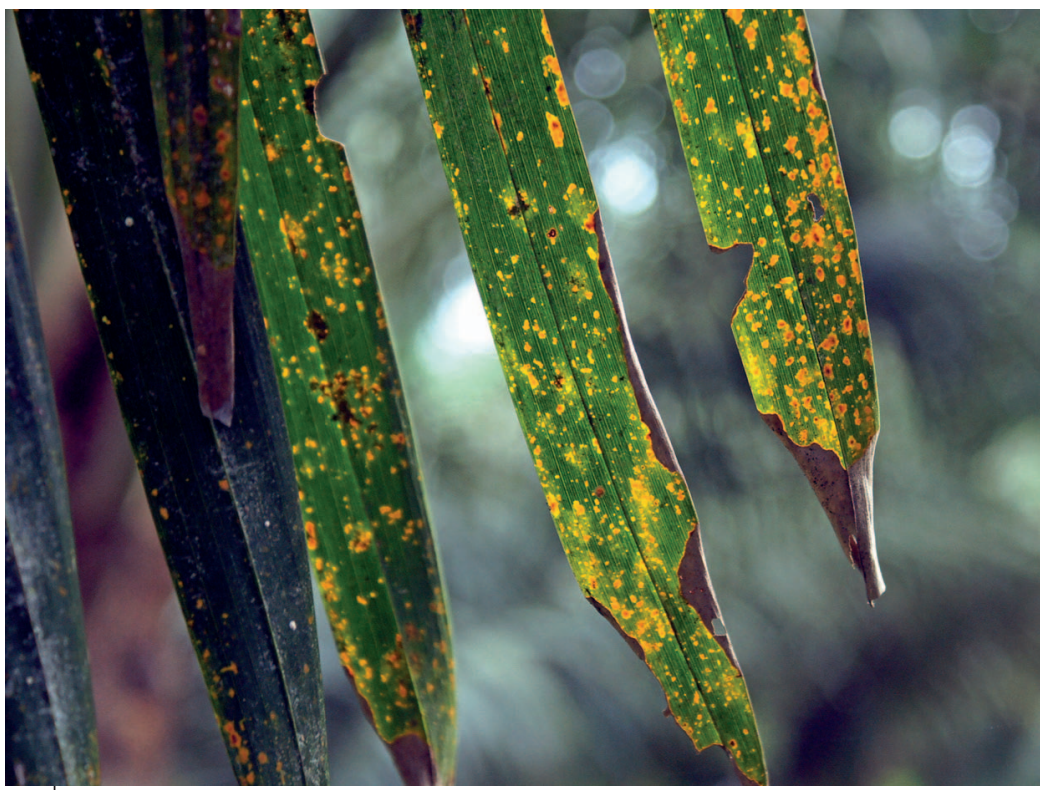

Figure 43.2.

Taches orangées caractéristiques de la déficience potassique. 
Le palmier à huile en plantation villageoise

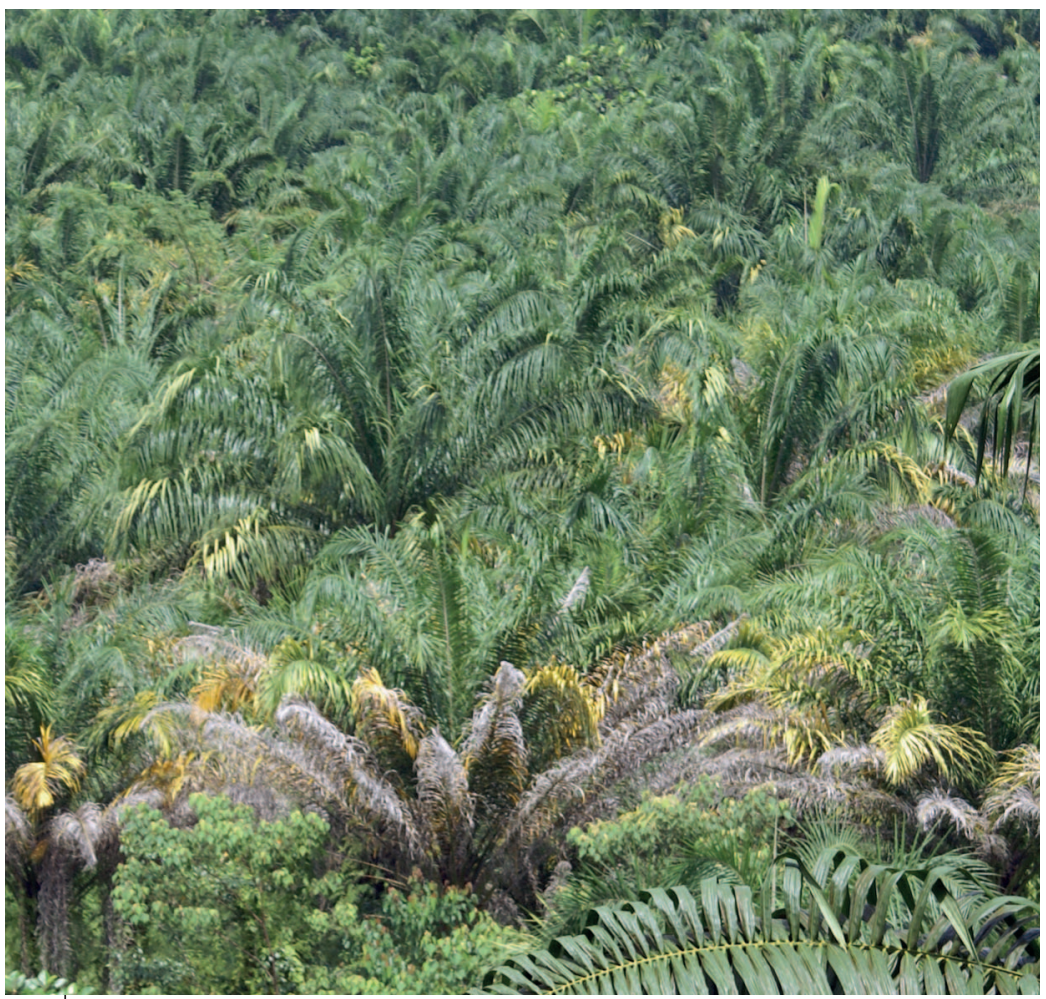

Figure 43.3.

Plantation adulte sévèrement atteinte de déficience potassique. 

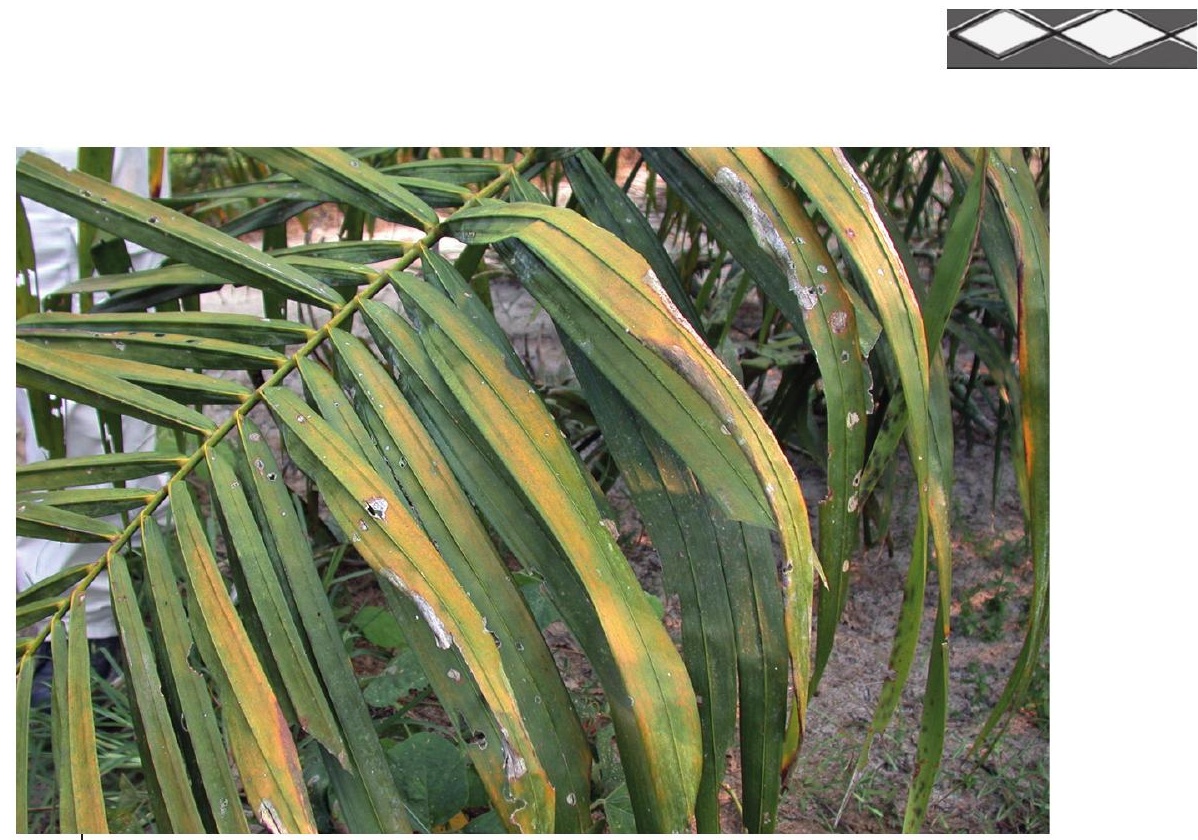

Figure 43.4.

Décoloration caractéristique des folioles d'un arbre souffrant de déficience magnésienne.

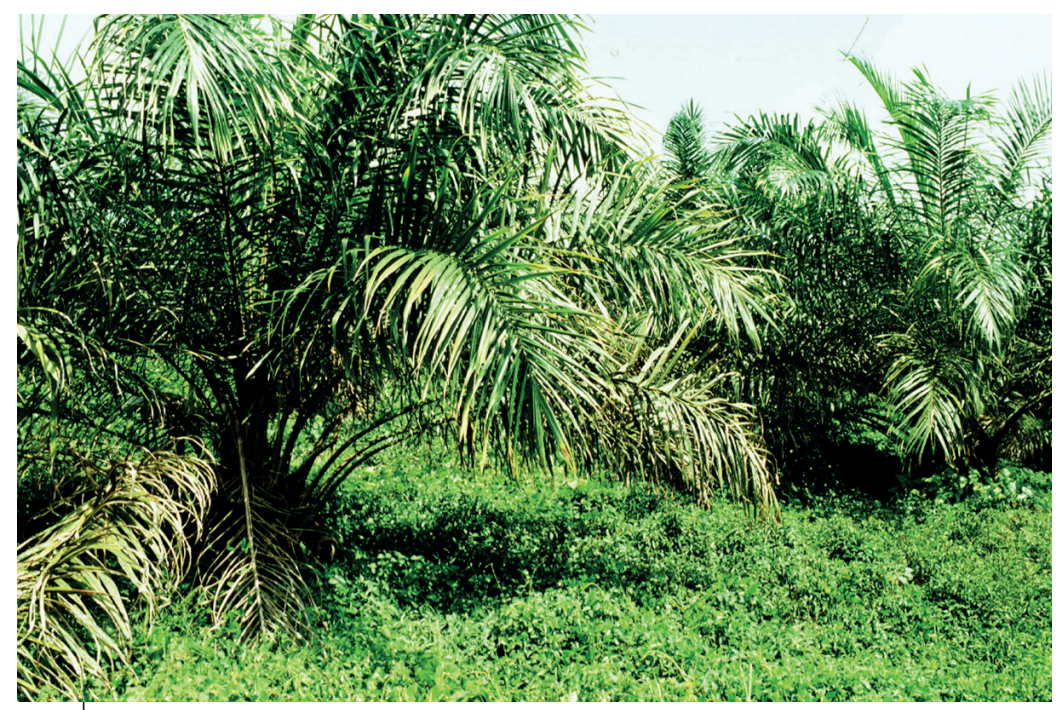

Figure 43.5.

Arbre de bordure souffrant de déficience magnésienne. 
Le palmier à huile en plantation villageoise

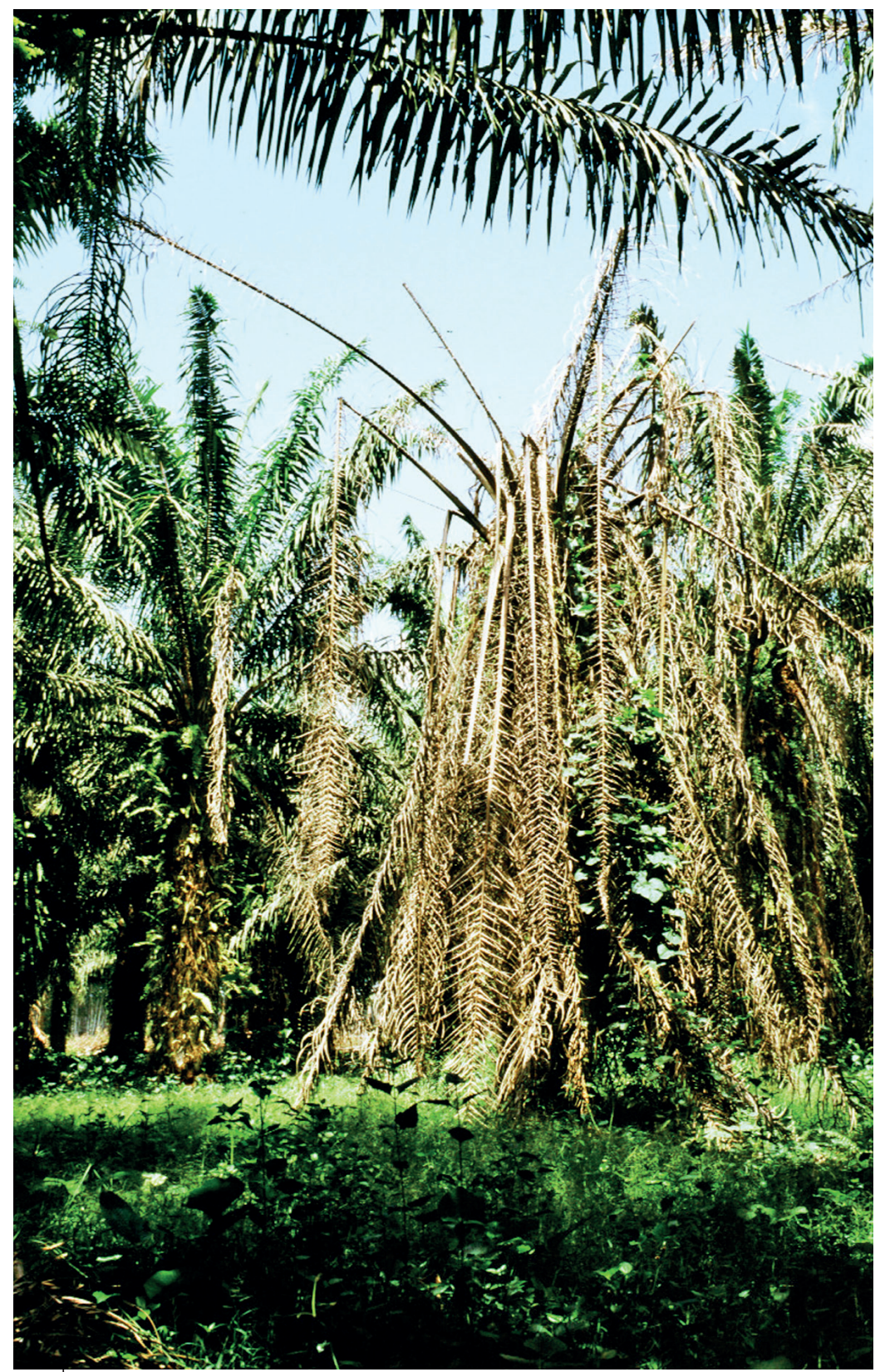

Figure 43.6.

Arbre frappé par la foudre. 


\section{Fiche 43. Déficiences nutritionnelles et dégâts provoqués par la foudre}

ID Stade sensible : pépinière, jeune culture.

Rare en plantation adulte

Déficience azotée

\section{Symptômes}

Décoloration uniforme des jeunes feuilles passant du vert sombre au vert jaune puis au jaune (figure 43.1). Ralentissement marqué de la croissance.

\section{Causes}

Mauvaise fertilisation, drainage défectueux, compétition avec des graminées, pauvreté naturelle du sol, mauvais développement de la plante de couverture, exportation des déchets organiques (feuilles, pétioles, folioles).

\section{Particularités}

La décoloration progresse vers les feuilles les plus âgées lorsque la déficience s'accentue.

\section{Remède}

Élimination de la cause (drainage, compétition, exportations), remettre en place la plante de couverture, application d'urée ou de sulfate d'ammonium.

IID Stade sensible : plantation adulte.

Possible en pépinière et en jeune culture

\section{Déficience potassique}

\section{Symptômes}

Décoloration diffuse vert jaune devenant vert pâle du bord des folioles. Taches orangées dites «orange spotting» devenant peu à peu confluentes (figure 43.2). Les feuilles atteintes se dessèchent précocement et prennent un port dressé.

\section{Causes}

Fertilisation insuffisante, pauvreté naturelle du sol (cas général des sols tropicaux), exportation des déchets organiques (feuilles, pétioles, folioles). 


\section{Particularités}

La réduction de la production de régimes se produit avant l'apparition des symptômes foliaires. Dans les cas grave, l'arbre prend un aspect desséché (figure 43.3). Ne pas confondre cette déficience nutritionnelle avec les symptômes de la cercosporiose. La déficience potassique favorise le développement de la fusariose.

\section{Recommandation}

Supprimer les exportations des déchets organiques.

Apporter des engrais organiques ou minéraux riches en potassium.

Mettre en place un suivi de la nutrition avec l'appui de la coopérative ou de la plantation commerciale la plus proche.

\section{IID Stade sensible : plantation adulte; possible en jeune culture}

\section{Déficience magnésienne}

\section{Symptômes}

Décoloration vert jaune puis jaune orange des folioles les plus exposées au soleil sur les palmes les plus âgées (figure 43.4). Les symptômes apparaissent plus facilement en bordure de parcelle (figure 43.5).

\section{Causes}

Excès de fertilisation potassique, sols pauvres en magnésium.

\section{Particularités}

Les symptômes de déficience apparaissent nettement avant que n'intervienne une baisse de la production. Les folioles ombragées par leurs voisines restent souvent vertes.

\section{Remède}

Réduire légèrement la fertilisation potassique et apporter un engrais magnésien. Mettre en place un suivi de la nutrition avec l'appui de la coopérative ou de la plantation commerciale la plus proche.

\section{Impact de foudre}

\section{Symptômes}

Feuilles médianes cassées au premier tiers, dessèchement rapide de la couronne basse, arrêt de l'allongement des flèches, mort rapide de l'arbre (figure 43.6).

\section{Particularités}

La présence de feuilles médianes cassées et sèches sur les arbres immédiatement voisins en regard de celui ou de ceux les plus touchés 
permet d'éviter de confondre avec un cas de fusariose typique. Les arbres qui n'ont pas pris l'impact survivent en général. Sur des plantations plus jeunes, plusieurs arbres, parfois distants, peuvent recevoir l'impact de la foudre en raison de son éclatement à l'approche du sol.

\section{Méthode de lutte}

Aucune.

\section{Attention}

Les dégâts provoqués au système foliaire par la foudre, la fusariose typique, une très forte sécheresse ou une déficience potassique très prononcée sont assez proches. Dans le doute, il convient de demander l'assistance d'un conseiller agricole. 



\section{Sécurité}

\section{Fiche 44. Rappels de sécurité}

\section{ID Équipements de protection}

Chaque planteur devrait posséder et revêtir pour son travail cet équipement de protection (figure 44.1).

Protection de la tête : cagoule (traitements phytosanitaires en hauteur).

Protection des yeux et du visage : lunettes de sécurité (traitements phytosanitaires).

Protection des voies respiratoires : masque simple pour les traitements phytosanitaires et les applications d'engrais.

Protection des mains : gants de protection adaptés au type de produit ou de travail (figure 44.2). Ne pas oublier de porter des gants fins de coton sous les gants de protection.

Protection du corps : vêtements spéciaux, tabliers (traitements phytosanitaires).

Protection des pieds : chaussures de sécurité, bottes (tous travaux).

\section{IID Les bons gestes}

Bien lire les étiquettes et les procédures et les appliquer.

Si nécessaire, demander l'explication des procédures dans sa langue maternelle.

Cesser immédiatement le travail en cas de malaise ou d'exposition dangereuse et se faire conduire au centre de santé le plus proche.

Signaler au médecin du centre tout phénomène allergique.

Les femmes enceintes ou allaitantes ne doivent pas approcher les produits phytosanitaires ou effectuer de traitements phytosanitaires.

Les enfants en bas âge ou mineurs doivent être éloignés de toute zone où sont stockés les produits phytosanitaires, les engrais, et des lieux où sont effectués les traitements phytosanitaires.

$\mathrm{Ne}$ jamais toucher les produits phytosanitaires ou les produits chimiques à mains nues. 


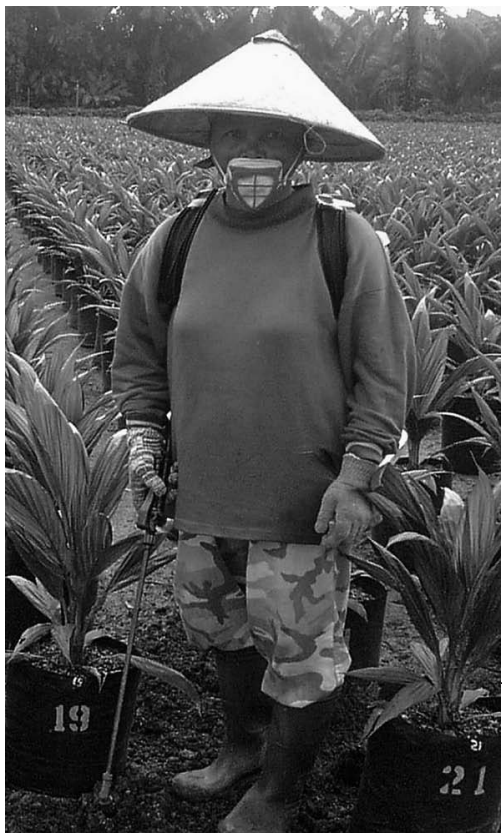

\section{Figure 44.1.}

Exemple de protection

pour une application de fongicide en pépinière.

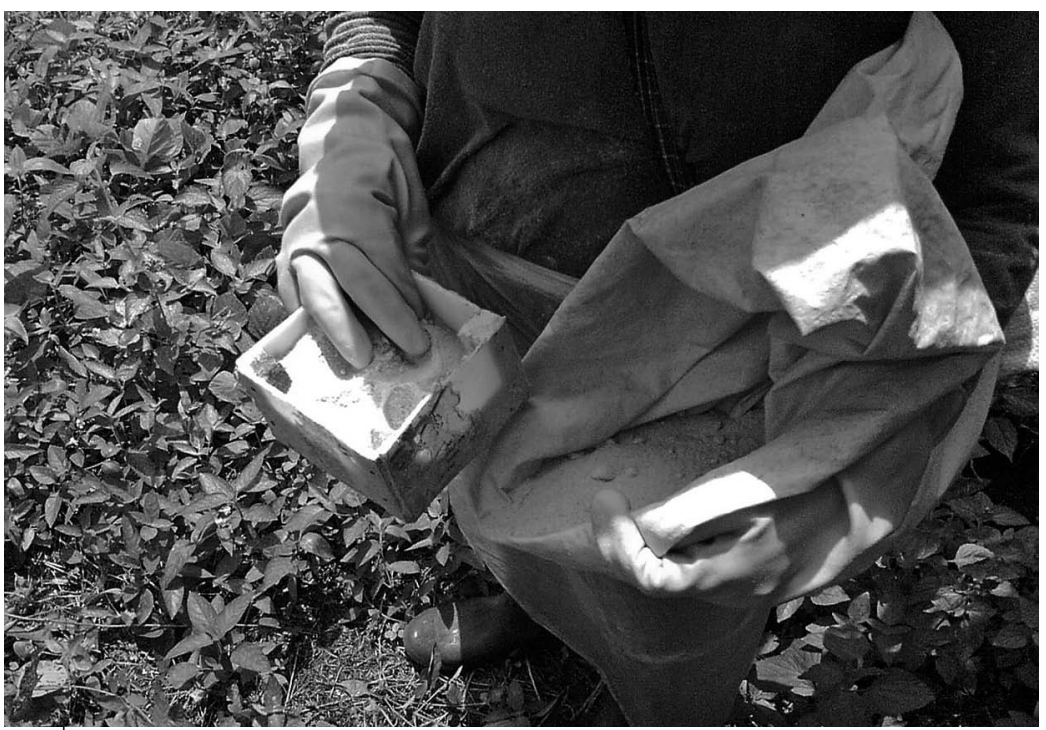

Figure 44.2.

Exemple de protection des mains

pour une application d'engrais. 
Ne porter aucune pièce d'appareil à la bouche (par exemple : pour déboucher une buse obstruée).

Toujours porter les vêtements de protection adéquats.

Ne pas fumer, boire ou manger pendant les opérations techniques.

Retirer ses vêtements de protection, se laver les mains et le visage avant de manger, de boire, de fumer ou d'aller aux toilettes.

Mettre ses gants de protection en premier lieu et les retirer en dernier lieu.

Nettoyer soigneusement ses vêtements de protection chaque jour.

Ne pas laver ses vêtements de protection avec les autres vêtements.

Stocker les vêtements de protection dans une pièce propre, sèche et bien ventilée, sans contact avec les autres vêtements.

Ne pas attendre que les équipements de protection soient complètement détériorés pour les remplacer.

$\mathrm{Ne}$ pas transporter ou entreposer des produits chimiques avec des denrées alimentaires.

Ne pas laver les appareils et équipements ayant servi à des applications de produits phytosanitaires ou chimiques dans une zone proche d'un lieu de prélèvement d'eau de boisson ou de bain.

Ne pas laisser les personnes à risque s'approcher des produits chimiques et des produits phytosanitaires dans les zones d'application en cours (enfants, femmes enceintes, adultes non informés).

$\mathrm{Ne}$ pas stocker de produits chimiques ou phytosanitaires dans des emballages sans étiquette ou marquage.

Brûler les emballages de produits chimiques ou phytosanitaires vides. Ne pas réutiliser les emballages de produits chimiques ou phytosanitaires en particulier pour la boisson ou les denrées alimentaires.

$\mathrm{Ne}$ jamais laisser les produits chimiques ou phytosanitaires et les équipements hors de leur lieu de stockage sans surveillance.

Enfin : APPLIQUEZ LES PROCEDURES. 



\section{Glossaire}

Abattis (Heap of felled trees) : ensemble des débris issus de l'opération d'abattage de la forêt.

Adventice (Weed) : se dit d'une plante, souvent indésirable, qui colonise un territoire à la suite d'une introduction accidentelle.

Anthracnose (Anthracnose) : maladie cryptogamique caractérisée par des taches brunes qui se développent sur l'extrémité des feuilles en prépépinière dans des conditions trop humides.

\section{Carpophore (Fruiting body) :} partie visible des champignons basidiomycètes.

Chicot (Stump) : vestige de la base du pétiole de la palme après la coupe de celle-ci. Ces chicots ont tendance à rester adhérents au stipe jusque vers $15-16$ ans, puis ils tombent au sol après leur complet dessèchement.

Coléoptile (Coleoptile) : enveloppe autour des premières feuilles. Lors du processus de germination de la graine de palmier, le coléoptile émerge avant les premières feuilles.

Compost (Compost) : mélange fermenté de débris organiques et de matières minéralisées. Dans le cas présent, mélange obtenu par la fermentation contrôlée des rafles et des effluents solides et liquides de l'huilerie de palme.

Conditions pédoclimatiques (pedoclimatic conditions) : ensemble des conditions de climat et de sol caractérisant la région.
Daba (Daba) : outil traditionnel du paysan ouest-africain dont la forme rappelle la houe.

\section{Démariage (Thinning out) :} Opération consistant à enlever les plantules surnuméraires qui se seront développées dans le sachet de prépépinière de façon à n'en laisser qu'une seule.

\section{Développement durable (Sustainable} development) : développement qui répond aux besoins des générations du présent sans compromettre la capacité des générations futures à répondre aux leurs (Rapport Bruntland, 1987). Le développement durable concilie les trois dimensions suivantes : économique, sociale et écologique.

Dura (Dura) : type de palmier dont le fruit est caractérisé par une coque épaisse entourant l'amande. Ce type est présent à plus de $95 \%$ dans les palmeraies naturelles. Son taux d'extraction est faible.

Élytre (Elytron) : aile antérieure dure et cornée des coléoptères. Elle ne sert pas au vol et recouvre l'aile postérieure à la façon d'un étui.

Feuille lancéolée (Lanceolate leaf) : feuille simple, au limbe plus long que large en forme de fer de lance. Les deux ou trois premières feuilles émises par la plantule de palmier à huile sont de ce type.

Feuilles primordiales (Primordial leaves) : petites feuilles faisant partie de la plumule.

Flèche (Spear) : feuille nouvellement émise et dont les folioles, encore collées ensemble, ne se sont pas encore épanouies. 
Forêt dégradée (Degraded forest) : forêt qui a subi une réduction de son couvert végétal et arboré soit par des causes naturelles soit par des causes humaines.

Fumure (Manuring, fertilisation) : engraissement d'un champ par du fumier. Par extension, engraissement d'un champ par tout type de fertilisant.

Homme-jour : unité de mesure correspondant au travail d'une personne pendant un jour, la durée du jour de travail étant fixée par la loi ou par un accord d'entreprise.

Humifère (Humus - bearing) : caractérise l'horizon du sol riche en humus.

Inflorescence (Inflorescence) : ensemble des fleurs regroupées sur une tige.

Jachère (Fallow) : état d'une terre cultivable qu'on laisse temporairement reposer, sans culture, pour restaurer sa fertilité.

Latérite (Laterite) : terre rouge riche en oxyde de fer développant souvent de véritables cuirasses impropres à la culture fréquente dans les pays tropicaux humides.

Limbe (Lamina) : partie large et étalée de la feuille.

Légitimité (Legitimacy) : dans ce contexte, qualité des semences qui doivent être issues de fécondation artificielle et produites dans des conditions qui assurent une absence de contamination (absence de types non désirés comme le dura ou le pisifera, absence de graines de fécondation libre, etc.).

Lutte biologique (Biological control) : exploitation par l'homme, à son profit, de la relation naturelle entre un organisme indésirable (ravageur, agent pathogène, etc.) qui est la cible et un auxiliaire de lutte qui est le plus souvent un parasite, un prédateur ou un pathogène de l'espèce cible. Il s'agit d'une méthode qui n'utilise pas de produits de synthèse.

Nymphose (Nymphosis) : état d'un insecte au second stade de sa métamorphose au cours de laquelle l'insecte se transforme en nymphe.

Palmier anormal (Abnormal palm tree) : palmier dont le feuillage ou le port est très éloigné de la conformation standard. Sa capacité de production est généralement réduite.

Palmier consanguin (Inbreed palm tree) : palmier issu d'un croisement entre deux individus apparentés. Le palmier à huile est très sensible à la consanguinité qui affecte fortement son potentiel de production.

Palmier illégitime (Illegitimate palm tree) : dans une population, palmier qui n'est pas issu des croisements qui la composent. Lincertitude de cette origine remet en question son potentiel de production.

Pédoncule (Peduncle) : support du régime de palme, il est inséré à l'aisselle de la feuille. Il est plus ou moins facile d'accès selon sa longueur et la morphologie du système foliaire.

Pétiole (Petiole) : partie de la feuille reliant le rachis au stipe.

Pisifera (Pisifera) : type de palmier dont le fruit est caractérisé par une absence de coque autour de l'amande. Ce type est généralement stérile femelle et ne produit que des rafles sans fruit développé.

Plumule (Plumelet) : partie du germe qui deviendra la tige. 
Pseudobulbe (pseudobulb) : chez les jeunes palmiers, partie charnue de la plante sur laquelle les feuilles sont fixées et d'où partent les racines. Chez les palmiers adultes, partie renflée du stipe située à sa base d'où partent les racines.

Rachis (Rachis) : partie axiale de la feuille du palmier qui porte les folioles. Partie axiale du régime de fruits du palmier qui porte les épillets.

Radicule (Radicle) : racine primordiale de la plantule.

Rafle (Empty bunch) : partie du régime de palme obtenu après le passage de ceux-ci dans l'égrappoir. La rafle est constituée par le rachis du régime et des épillets débarrassés de leurs fruits.

Ravageur (Pest) : se dit d'un animal qui provoque des dégâts importants sur les cultures notamment pour se nourrir.

Régime de palme (Bunch) : chez le palmier à huile, nom communément donné à l'inflorescence femelle lorsque les fleurs ont commencé à se développer en fruits après l'anthèse.

Sol argileux (Clay soil) : sol riche en argile, souvent compact, lourd, se ressuyant mal. Présence de fentes de rétractation lorsqu'il s'assèche.
Sol gravillonnaire (Gravel soil) : sol riche en graviers souvent issus de la dégradation d'une cuirasse ferralitique.

Spire (Helix) : ligne imaginaire qui suit le positionnement des feuilles émises successivement. La spire la plus utilisée chez le palmier est la spire d'ordre 8 . Il y en a donc $8 \mathrm{chez}$ le palmier à huile. Elle «tourne» indifféremment à droite ou à gauche. La spire 1 permet de repérer les feuilles 1, 9, 17, 25, 34 et 43 dans la couronne.

Stipe (Stipe, stalk) : tige ligneuse des plantes monocotylédones arborescentes et des fougères. Cette tige ne se ramifie pas chez le palmier à huile, sauf accident de végétation.

Tenera (Tenera) : type de palmier issu du croisement entre un dura et un pisifera (voir ces entrées). C'est ce type d'arbre qui est utilisé dans toutes les exploitations modernes qu'elles soient commerciales ou villageoises.

Tine (Tine) : nom donné en Afrique francophone à des récipients métalliques de récupération, le plus souvent d'une capacité d'environ 20 à 25 litres. Par extension, il concerne aussi des récipients de matière plastique de même capacité. 



\section{Pour en savoir plus}

Jacquemard J.C., 2011. Le palmier à huile. Collection Agricultures tropicales en poche, Quæ, CTA et Presses agronomiques de Gembloux.

Anon., 2002. Mémento de l'agronome. Cirad, CTA, Gret et MAE.

\section{Sur les productions associées}

Fellows P., 2011. Le conditionnement des produits agricoles. Collection Agrodok. CTA et Agromisa.

Ngo-Samnick E.L., 2011. Production améliorée du bananier plantain. Collection Pro-Agro. CTA et ISF Cameroun.

Van Lidth de Jeuge J., 2004. Identification des dégâts causés aux cultures par les maladies, les animaux nuisibles et les carences minérales. Collection Agrodok. Agromisa, CTA.

Degras L., 1994. L'igname. Collection : Le technicien d'agriculture. ACCT, CTA et Maisonneuve et Larose.

\section{Sur l'agriculture familiale}

Touzard I. et Ferraton N., 2009. Comprendre l'agriculture familiale - Diagnostic des systèmes de production. Collection Agricultures tropicales en poche, Quæ, CTA et Presses agronomiques de Gembloux.

Dupriez H., 2006. Agriculture tropicale et exploitations familiales d'Afrique. Collection Terres et Vie, CTA et Terres et Vie.

Dugué M.J., Pesche D., Le Coq J.F., 2012. Appuyer les organisations de producteurs. Collection Agricultures tropicales en poche, Quæ, CTA et Presses agronomiques de Gembloux.

Nacro S., 2011. Comment assurer une facilitation de qualité aux producteurs agricoles à travers les champs-écoles. CTA et Harmattan Burkina. 
Photo de couverture : Épandage d'engrais en plantation de palmier à huile par une paysanne équipée de gants et de bottes

Illustrations et photos : ( ) J.C. Jacquemard

Édition : Claire Parmentier, Presses agronomiques de Gembloux

Maquette : Patricia Doucet

Infographie : Editions Quæ

Mise en page : Hélène Bonnet

Impression : Jouve 


L'huile de palme reste la première source de corps gras végétal sur le marché mondial, la culture du palmier à huile est donc au cœur du développement des pays de la ceinture tropicale. La filière agro-industrielle du palmier à huile repose en grande partie sur des plantations villageoises : plus de $50 \%$ des surfaces plantées dans plusieurs pays (Cameroun, Côte d'Ivoire, Ghana, Nigeria, Sierra Leone, Thaillande), plus de $40 \%$ en Malaisie et en Indonésie. Cet ouvrage complète "Le palmier à huile ", publié en 2011 dans cette même collection et du même auteur, et s'adresse plus particulièrement aux planteurs de palmier à huile des plantations villageoises. II fournit les bases de l'installation et du développement de la palmeraie, tout en restant en accord avec les objectifs d'un développement durable. Il est organisé en 44 fiches couvrant tous les aspects : prépépinière, pépinière, création de la plantation, jeunes cultures, plantation en rapport, récolte des régimes, principaux accidents (maladies, ravageurs, nutrition). Des préconisations essentielles à la sécurité sont enfin rappelées. Ainsi, cet ouvrage constitue une référence pour les itinéraires techniques du palmier à huile, quel qu'en soit le mode d'exploitation.

Rédigé dans un langage clair, très précis et abondamment illustré par des schémas et des photos, cet ouvrage est surtout destiné aux planteurs, aux agents d'encadrement, aux formateurs et aux décideurs de projets villageois de cette filière.

Jean-Charles Jacquemard, ingénieur agronome et ancien chercheur au Cirad, est spécialiste du palmier à huile. Pendant quarante ans, en Indonésie et en Afrique, il a été impliqué dans les filières villageoise et agro-industrielle. Membre titulaire du comité d'experts chargé de la rédaction des principes et critères RSPO, il a été également membre du comité scientifique de l'Association africaine pour le développement du palmier à huile qui a soutenu l'essor des plantations villageoises en Afrique de l'Ouest et du Centre.
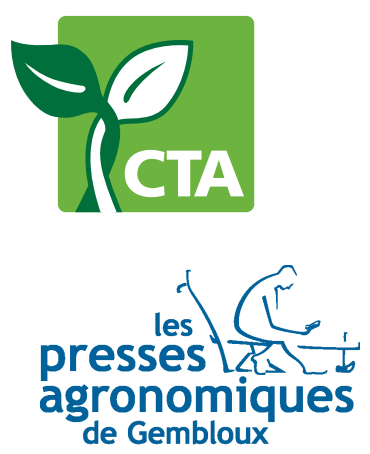

$18,30 €$

ISBN : 978-2-7592-1986-5

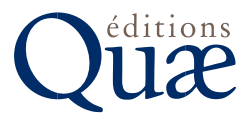

